\title{
Synthesis of Arabinoxylan Oligosaccharides by Pre-Activation-Based Iterative Glycosylations
}

\author{
Underlin, Emilie Nørmølle; Böhm, Maximilian Felix; Madsen, Robert
}

Published in:

Journal of organic chemistry

Link to article, DOI:

10.1021/acs.joc.9b02529

Publication date:

2019

Document Version

Peer reviewed version

Link back to DTU Orbit

Citation (APA):

Underlin, E. N., Böhm, M. F., \& Madsen, R. (2019). Synthesis of Arabinoxylan Oligosaccharides by PreActivation-Based Iterative Glycosylations. Journal of organic chemistry, 84(24), 16036-16054.

https://doi.org/10.1021/acs.joc.9b02529

\section{General rights}

Copyright and moral rights for the publications made accessible in the public portal are retained by the authors and/or other copyright owners and it is a condition of accessing publications that users recognise and abide by the legal requirements associated with these rights.

- Users may download and print one copy of any publication from the public portal for the purpose of private study or research.

- You may not further distribute the material or use it for any profit-making activity or commercial gain

- You may freely distribute the URL identifying the publication in the public portal

If you believe that this document breaches copyright please contact us providing details, and we will remove access to the work immediately and investigate your claim. 


\section{Article}

\section{Synthesis of Arabinoxylan Oligosaccharides by Pre-Activation-Based Iterative Glycosylations}

Emilie N. Underlin, Maximilian Böhm, and Robert Madsen

J. Org. Chem., Just Accepted Manuscript • DOI: 10.1021/acs.joc.9b02529 • Publication Date (Web): 25 Nov 2019

Downloaded from pubs.acs.org on December 3, 2019

\section{Just Accepted}

"Just Accepted" manuscripts have been peer-reviewed and accepted for publication. They are posted online prior to technical editing, formatting for publication and author proofing. The American Chemical Society provides "Just Accepted" as a service to the research community to expedite the dissemination of scientific material as soon as possible after acceptance. "Just Accepted" manuscripts appear in full in PDF format accompanied by an HTML abstract. "Just Accepted" manuscripts have been fully peer reviewed, but should not be considered the official version of record. They are citable by the Digital Object Identifier (DOI®). "Just Accepted" is an optional service offered to authors. Therefore, the "Just Accepted" Web site may not include all articles that will be published in the journal. After a manuscript is technically edited and formatted, it will be removed from the "Just Accepted" Web site and published as an ASAP article. Note that technical editing may introduce minor changes to the manuscript text and/or graphics which could affect content, and all legal disclaimers and ethical guidelines that apply to the journal pertain. ACS cannot be held responsible for errors or consequences arising from the use of information contained in these "Just Accepted" manuscripts. 


\title{
Synthesis of Arabinoxylan Oligosaccharides by Pre-Activation-Based Iterative Glycosylations
}

\author{
Emilie N. Underlin, Maximilian Böhm, and Robert Madsen* \\ Department of Chemistry, Technical University of Denmark, 2800 Kgs. Lyngby, Denmark \\ E-mail: rm@kemi.dtu.dk
}

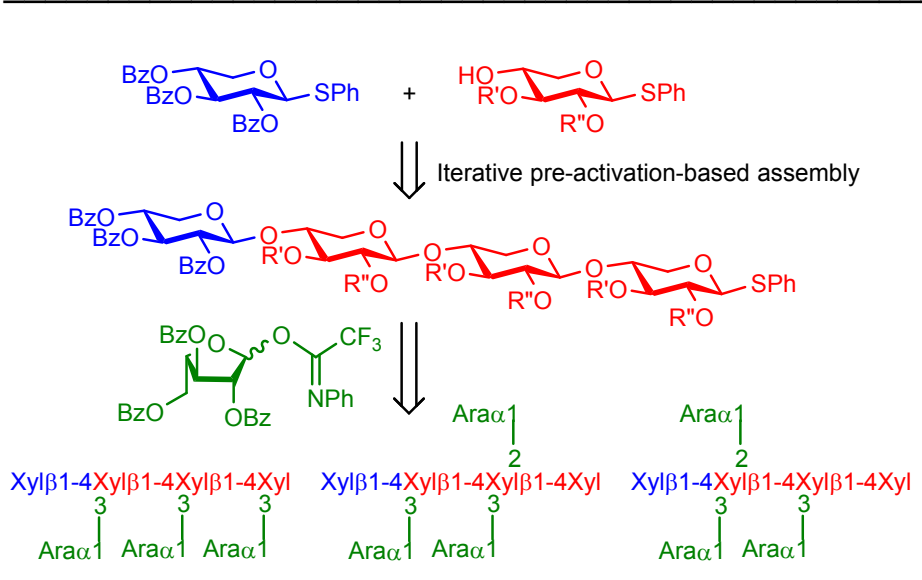

\begin{abstract}
A concise synthetic strategy has been developed for assembling densely substituted arabinoxylan oligosaccharides, which are valuable substrates for characterizing hemicellulosedegrading enzymes. The xylan backbone has been prepared by an iterative pre-activation-based glycosylation approach with phenyl thioglycosides. The pre-activation has been performed with in situ generated $p$-nitrobenzenesulfenyl triflate prior to addition of the acceptor. The glycosylation temperature was shown to have an important impact on the yield of the coupling. The arabinose substituents have been introduced in one high-yielding glycosylation with a $N$ phenyl trifluoroacetimidate donor. The strategy has been successfully employed for the
\end{abstract}


synthesis of three heptasaccharides in seven steps and overall yields of $24-36 \%$ from the corresponding monosaccharide building blocks.

\section{INTRODUCTION}

The hemicellulose polysaccharides comprise $15-35 \%$ by weight of the plant cell wall which is the main source of lignocellulosic biomass. ${ }^{1}$ The largest group of polysaccharides in hemicellulose are the xylans which are characterized by a common backbone of $\beta(1 \rightarrow 4)$-linked xylopyranosides. ${ }^{1}$ The xylan structures are highly complex and heterogeneous due to the differences in the substitution patterns. One of the most abundant xylan subclasses are the arabinoxylans where some of the xylose units are substituted with L-arabinofuranosyl residues through either single substitution by $\alpha(1 \rightarrow 2)$ or $\alpha(1 \rightarrow 3)$ linkages or double substitution by both linkages. ${ }^{1,2}$

The arabinoxylans are dietary fibers that are not hydrolyzed by the digestive enzymes. ${ }^{3}$ Instead they are fermented by microbes in the gut and in this way influences the composition and abundance of the colonic microbiota. ${ }^{3}$ The result is a prebiotic effect where the health of the host is benefitted. Arabinoxylans have been shown to 
strengthen the immune system and to lower the risk for obesity, type 2 diabetes, colon cancer and cardiovascular diseases. ${ }^{3}$ The physiological functions, however, are strongly connected to the structure of the arabinoxylans, i.e. the length of the xylan backbone and the distribution of the arabinose units. ${ }^{3}$ Besides food applications arabinoxylans are also used as a renewable source to prepare biodegradable materials such as films and hydrogels. ${ }^{1,4}$

Arabinoxylans are cleaved by glycosyl hydrolases $(\mathrm{GH})$ to form smaller saccharides. The hydrolysis of the xylose linkages can be achieved with $\mathrm{GH} 10$ and $\mathrm{GH} 11$ xylanases, which prefer several unsubstituted xylose units, and with GH5 xylanases where an arabinose substituent is allowed close to the cleavage site. ${ }^{5}$ Hydrolysis of the arabinose residues can be performed with $\mathrm{GH} 43, \mathrm{GH} 51$ and $\mathrm{GH} 62$ arabinofuranosidases where some of the enzymes exclusively cleave arabinoses linked to the 2 or the 3 position of single substituted xylose residues, whereas others are able to remove the pentose from disubstituted xylose moieties. ${ }^{5,6}$ All the enzyme classes, however, suffer from poor access in the densely substituted regions of arabinoxylans and the difficulties are 
further complicated by the lack of well-defined substrates with multiple arabinose

substituents.

Arabinoxylan-degrading enzymes are usually investigated with rather heterogeneous

polysaccharides isolated from natural sources by suitable pretreatment procedures. A

few saccharides containing $2-4$ xylose units and $1-2$ arabinose residues can be

isolated from enzymatic degradation of arabinoxylans, ${ }^{7}$ but these simple substrates do

not resemble the more densely substituted regions. Thus, well-defined xylans with

several arabinose substituents would be valuable tools for mapping the active site of

enzymes implicated in arabinoxylan degradation and to understand the interplay

between different enzymes in order to achieve the most efficient deconstruction.

Chemical synthesis offers the possibility to prepare pure and well-defined

oligosaccharides, but the arabinoxylans have so far received relatively little attention.

Xylobiose has been glycosylated with one or two equiv. of an arabinofuranosyl donor to

afford tri- and tetrasaccharides. ${ }^{8}$ Recently, arabinoxylans with up to 6 xylose residues

and $1-2$ arabinose units were prepared by solid-phase synthesis and used for 
determining the epitopes for monoclonal antibodies and the substrate specificity for xylanases and arabinofuranosidases. ${ }^{9}$ In one case, two arabinoses were glycosylated onto the 2 and the 3 position of the same xylose residue although a very low yield was obtained illustrating the challenge of producing more densely substituted arabinoxylans. Linear xylans, on the other hand, where no additional substituents are present, are more readily assembled by either stepwise or blockwise approaches ${ }^{10}$ and so far linear xylans up to decaxylans have been prepared, ${ }^{11}$ which includes an approach by cleaving the hydroxymethyl group of glucans ${ }^{12}$ and the assembly of $\mathcal{S}$ linked oligoxylans. ${ }^{13}$

Herein, we report a novel pre-activation-based synthetic strategy for assembling arabinoxylans, which has led to the preparation of heptasaccharides $1-3$ with three arabinose substituents (Scheme 1). A tetraxylan is chosen as the backbone since this constitutes a sufficient length to give the necessary enzyme activities.5,9a The three arabinose substituents are connected to either a xylotriose unit (as in 1) or a xylobiose moiety (as in $\mathbf{2}$ and $\mathbf{3}$ ) in order to resemble the densely substituted region. The 
preparation of $1-3$ extends our work on the synthesis of plant cell wall components

where we have previously assembled oligomers of rhamnogalacturonan I and

homogalacturonan by using $n$-pentenyl glycosides as glycosyl donors. ${ }^{14}$

\section{Scheme 1. Structures of Arabinoxylans 1 - 3 and Retrosynthetic Strategy}

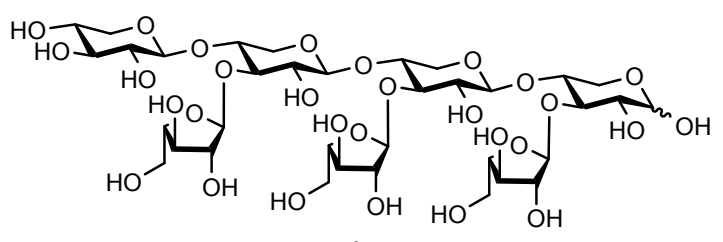

1

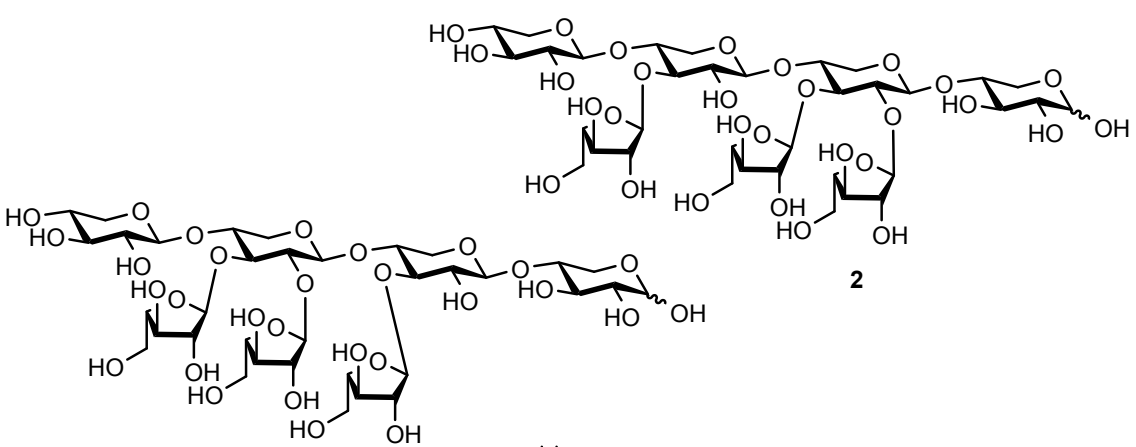

3

$\downarrow$

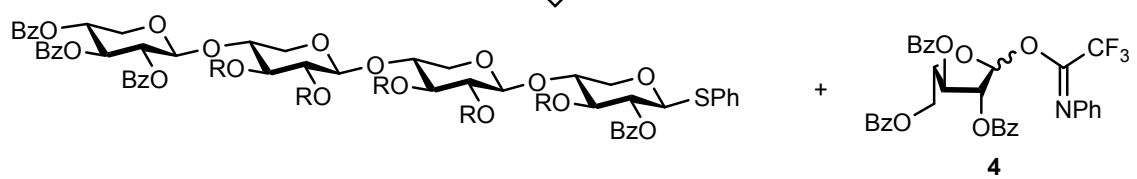

Pre-activation-based assembly

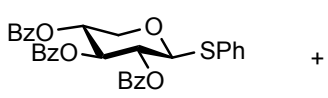

ROL

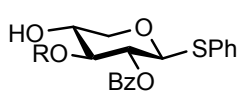

5

\section{RESULTS AND DISCUSSION}


The recent solid-phase synthesis of arabinoxylans was achieved by a stepwise approach where one xylose or arabinose unit was glycosylated at a time onto the growing oligosaccharide chain followed by cleavage from the resin and global deprotection. ${ }^{9} \mathrm{Up}$ to ten equiv. of a xylopyranosyl phosphate (prepared from the corresponding tolyl thioglycoside) or an ethyl 1thioarabinofuranoside was used as the donor for each glycosylation reaction. We opted for a solution-phase strategy involving fewer steps where all the arabinose units are introduced in one coupling reaction (Scheme 1). The linear xylan backbone will be prepared by a pre-activationbased synthesis ${ }^{15}$ from the appropriate phenyl 1-thioxylopyranosides where deprotection steps will not be necessary after each glycosylation reaction. ${ }^{16}$ The pre-activation-based thioglycoside protocol has previously been used for coupling of a variety of monosaccharides ${ }^{15,17}$ including arabinofuranosides, ${ }^{18}$ but it has so far not been applied to the coupling of xylose units. The benzoyl group will serve as a permanent protecting group for the xylose residues since it allows for neighboring group participation from the 2 position to afford the $\beta$-linkage in the glycosylations. The arabinose units will also be protected with benzoyl groups and the corresponding $N$-phenyl trifluoroacetimidate $\mathbf{4}$ will serve as the donor since it has previously given high yields in the coupling with different secondary alcohols. ${ }^{19}$

\section{Scheme 2. Synthesis of Building Blocks 5 and 6}




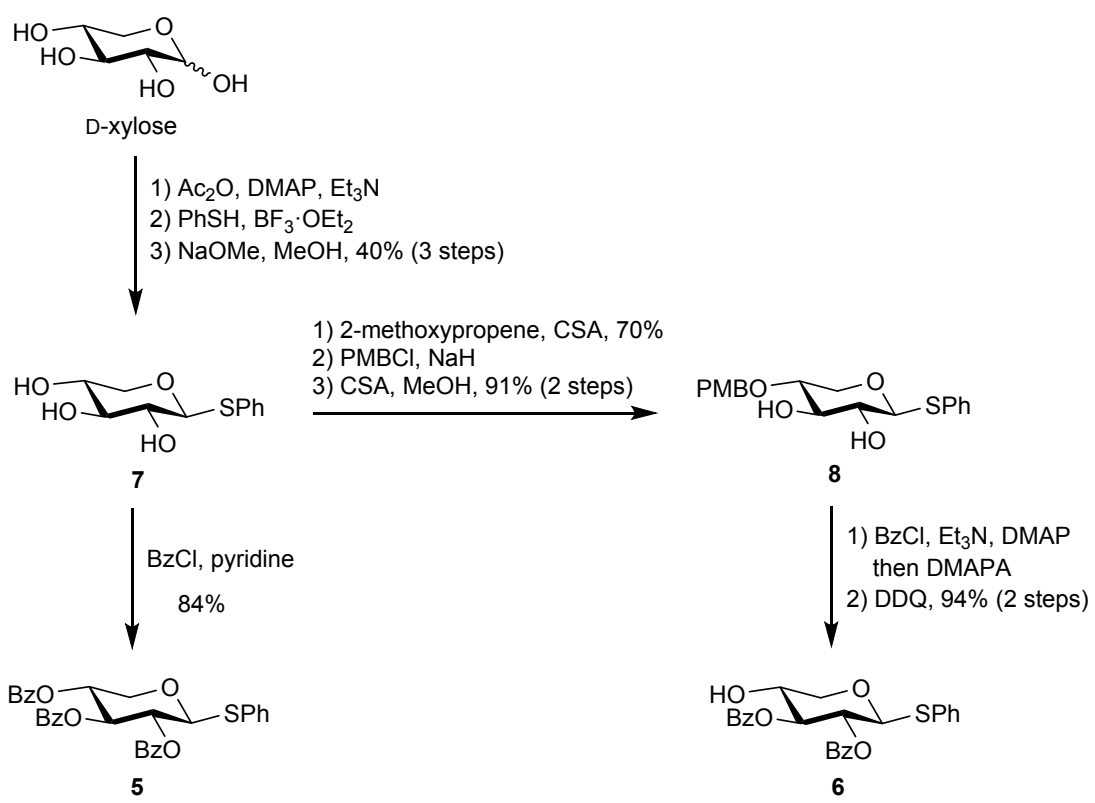

Several phenyl 1-thioxylopyranoside building blocks were required for the xylan assembly by the pre-activation-based approach. This involves fully benzoylated glycoside 5, 2,3-di-Obenzoylated glycoside 6 and two glycosides with different protecting groups at position 2 and 3 . The building blocks were all prepared from phenyl 1-thio- $\beta$-D-xylopyranoside (7), which is available from xylose in three simple steps involving peracetylation in the presence of 4- $(N, N-$ dimethylamino)pyridine (DMAP), thiolation and deacetylation ${ }^{20}$ with no purification of the intermediates (Scheme 2). Perbenzoylation of 7 then afforded tribenzoate 5 while the preparation of dibenzoate 6 required selective transformations of the hydroxy groups. In xylopyranosides the three hydroxy groups are all equatorial and similar in reactivity making regioselective reactions a particular challenge. ${ }^{21}$ One of the most effective procedures is to block the 2 and the 3 position with an isopropylidene group, which affords only minor amounts of the corresponding 3,4-acetal. ${ }^{22}$ Thus, treatment of triol 7 with 2-methoxyprop-1-ene and camphorsulfonic acid (CSA) led to the acetonide at position 2 and 3 as the major product. 
Subsequently, a para-methoxybenzyl group (PMB) was installed at position 4 and the acetonide was removed under acidic conditions to provide diol $8 .^{23}$ The hydroxy groups were benzoylated and excess benzoyl chloride removed with 3-(dimethylamino)-1-propylamine (DMAPA). ${ }^{24}$ Without further purification the crude product was subjected to PMB deprotection with 2,3dichloro-5,6-dicyano-1,4-benzoquinone (DDQ) to afford dibenzoate 6.

\section{Scheme 3. Synthesis of Building Blocks 8 and 10}

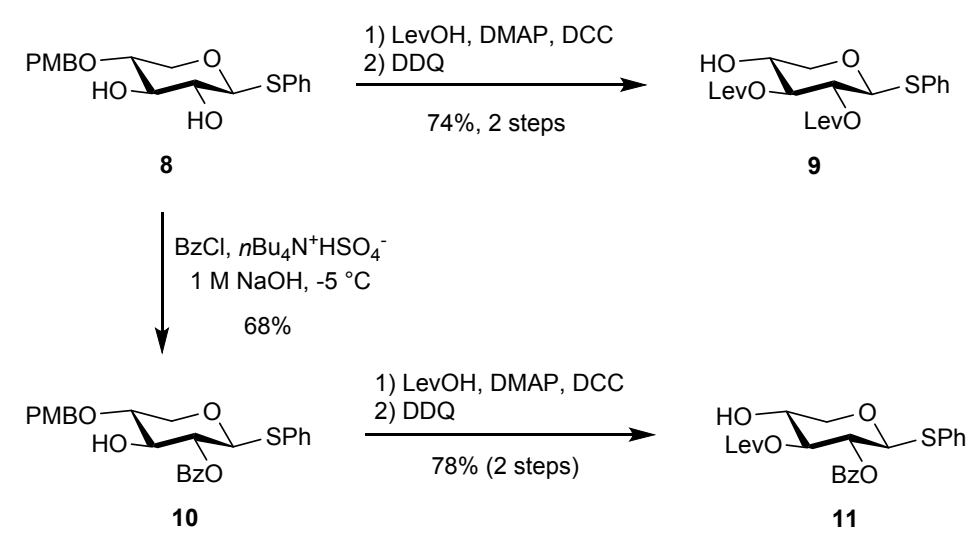

The two remaining building blocks required a different protecting group to be installed at position 3 as well as at position 2 and 3 since these sites will be linked to the arabinose units. The temporary protecting group should be an ester to ensure neighboring group participation in the glycosylation, but should at the same time be removable in the presence of the benzoates. A chloroacetate was first considered and installed selectively at position 3 , but the following glycosylation with $\mathbf{5}$ under the pre-activation protocol only furnished a moderate yield of the disaccharide. This was attributed to the use of a silver salt in the glycosylation and a levulinyl (Lev) group was therefore chosen instead. $N, N$ '-Dicyclohexylcarbodiimide (DCC) coupling of diol 8 with levulinic acid followed by direct treatment with DDQ furnished dilevulinate 9 
(Scheme 3). For the selective introduction of the temporary protecting group, diol $\mathbf{8}$ was first subjected to regioselective benzoylation at position 2 . With benzoyl chloride in pyridine the esterification was slow and actually produced the dibenzoate as the main product. However, when diol 8 was submitted to 1.3 equiv. of benzoyl chloride in a mixture of $\mathrm{CH}_{2} \mathrm{Cl}_{2}$ and $1 \mathrm{M}$ $\mathrm{NaOH}$ under phase transfer catalysis, ${ }^{25}$ 2-benzoate 10 was obtained in $68 \%$ yield with only minor amounts of the corresponding 3-benzoate as a byproduct. Subsequent esterification with levulinic acid and deprotection of the PMB group gave the desired 3-levulinate 11.

The stage was now set for assembling the xylan backbone by the pre-activation-based protocol. The original procedure with tolylthio glycosides called for activation of the donor with $p$-toluenesulfenyl chloride and silver triflate at a temperature of $-60{ }^{\circ} \mathrm{C}$ to generate the reactive electrophile. ${ }^{16}$ The acceptor was then added followed by stirring for $15 \mathrm{~min}$ and warming to rt. The procedure was later on modified with the formation of the reactive electrophile at $-78^{\circ} \mathrm{C}$ followed by adding the acceptor and raising the temperature to $\mathrm{rt}^{17}$ Using this latest protocol, donor 5 was coupled to acceptor $\mathbf{6}$ with phenylsulfenyl chloride and silver triflate ${ }^{26}$ to give disaccharide 12 in 64\% yield (Scheme 4, Method A). The coupling could also be carried out in a one-pot iterative manner ${ }^{16}$ by adding more promoter and acceptor to furnish trisaccharide $\mathbf{1 3}$ although the yield in this case was only $34 \%$ since disaccharide 12 was also formed in $59 \%$ yield. Attempts to improve the glycosylations by using $\mathrm{Me}_{2} \mathrm{~S}_{2} / \mathrm{Tf}_{2} \mathrm{O}^{27}$ or 1-benzenesulfinyl piperidine $/ \mathrm{Tf}_{2} \mathrm{O}^{28}$ as the promoter were unsuccessful and only led to a mixture of products. However, when $p$-nitrobenzenesulfenyl chloride and silver triflate ${ }^{29}$ was employed as the promoter, the yield of $\mathbf{1 2}$ and $\mathbf{1 3}$ in the two reactions increased to 79 and 69\%, respectively. $p$ Nitrobenzenesulfenyl chloride is commercially available as opposed to phenylsulfenyl chloride and $p$-toluenesulfenyl chloride which are prepared from the corresponding thiols with sulfuryl 
chloride. In addition, $p$-nitrobenzenesulfenyl chloride is a solid making this reagent procedurally more convenient to deliver while the other two sulfenyl chlorides are liquids. The quality of commercial $p$-nitrobenzenesulfenyl chloride, however, varied and we obtained lower glycosylation yields when using light brown samples with a mp of $\sim 43{ }^{\circ} \mathrm{C}$. Attempts to recrystallize the compound failed, but sublimation yielded material with a light yellow color and a mp of $47-51^{\circ} \mathrm{C}$ which could be stored at $5{ }^{\circ} \mathrm{C}$ for months. When $p$-nitrobenzenesulfenyl chloride purified by sublimation was used, the yields in the glycosylations were consistent. During the couplings, the disulfide byproduct from the activation (i.e. $p \mathrm{NO}_{2} \mathrm{PhSSPh}$ ) precipitated and did therefore not take part in any additional reactions.

\section{Scheme 4. Synthesis of Saccharides 12 and 13}

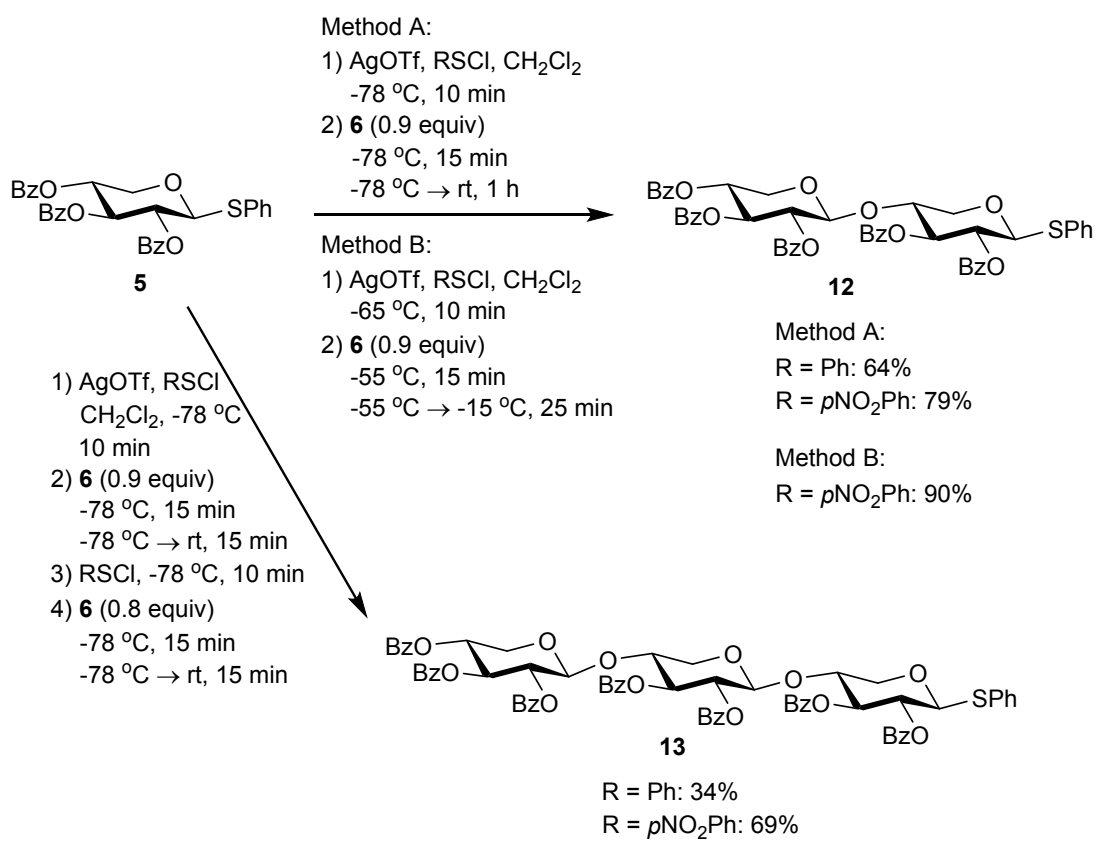

To further improve the glycosylation outcome the influence of the temperature was also investigated. These experiments were performed in a two-neck Schlenk flask that allowed for 
measuring the internal temperature in the reaction mixture at any time during the glycosylation. The activation of donor $\mathbf{5}$ was observed to be quick and goes to completion in less than $10 \mathrm{~min}$ at $-78^{\circ} \mathrm{C}$ according to $\mathrm{TLC}$ analysis. This is in accordance with investigations on the activation of similar thioglycosides under these conditions. ${ }^{30}$ However, in the ensuing glycosylation reaction after adding acceptor $\mathbf{6}$ the reaction temperature becomes important. If the temperature is kept at $-78{ }^{\circ} \mathrm{C}$ the glycosylation is sluggish and an intermolecular aglycon transfer ${ }^{31}$ becomes a competing reaction leading to the regeneration of donor $5 .^{32}$ Under these conditions disaccharide 12 was only obtained in 54\% yield together with 10\% of donor 5 although full activation of the donor had been achieved. Thus, at very low temperature the hydroxy group and the sulfur in acceptor 6 are both able to serve as a nucleophile in the reaction with the donor. Further experiments revealed that the aglycon transfer was mainly observed when the temperature was kept below $-60{ }^{\circ} \mathrm{C}$. Therefore, three separate experiments were set up where acceptor 6 was added at $-55^{\circ} \mathrm{C}$ (Figure 1). In the first experiment, the temperature was immediately raised to rt over $1 \mathrm{~h}$ while in the two other experiments the temperature was first maintained at $-55^{\circ} \mathrm{C}$ for $10-15$ min before being raised. The first reaction gave $73 \%$ yield of disaccharide 12 whereas 85 and 90\% yield were obtained in the two other experiments (Figure 1 and Scheme 4, Method B). This is a notable difference and illustrates the significance of the temperature in securing an optimal coupling reaction. If the temperature is too low the aglycon transfer becomes a side reaction while if the temperature is too high the activated intermediate of donor $\mathbf{5}$ (which is mostly the corresponding 1,2-benzoxonium ion) ${ }^{33}$ presumably decomposes. Thus, a coupling temperature of $-55^{\circ} \mathrm{C}$ was included in the protocol in the following experiments. The reactions were moinitored by TLC at $-55^{\circ} \mathrm{C}$ and the temperature was only raised when the coupling had gone to completion. 


\section{Figure 1. Reaction Temperatures and Yields for Coupling of 5 and 6 to afford 12}

Using this optimized procedure donor $\mathbf{5}$ was then coupled to Lev-protected acceptor $\mathbf{1 1}$ to afford disaccharide 14 in $86 \%$ yield (Scheme 5). Unfortunately, when the same coupling was performed in a one-pot fashion with the further addition of acceptor $\mathbf{6}$, trisaccharide $\mathbf{1 5}$ was only obtained in $28 \%$ yield. The addition of 2,4,6-tri-tert-butylpyrimidine ${ }^{15,34}$ to mitigate the possible influence of the increasing amount of acid lowered the yield even further to $17 \%$. Instead, large amounts of unreacted acceptor $\mathbf{6}$ were recovered in both cases. The low yield was not caused by the inability of Lev-protected thioglycosides to serve as donors since the coupling between disaccharide donor $\mathbf{1 4}$ and acceptor $\mathbf{6}$ gave rise to trisaccharide $\mathbf{1 5}$ in 69\% yield. Possibly, the mediocre result is caused by the Lev group undergoing further transformations due to the longer reaction time in the one-pot protocol. Therefore, the xylan backbones in targets $\mathbf{1}-\mathbf{3}$ will be assembled by an iterative glycosylation approach with pre-activation where the glycosylation products once isolated will be immediately used as donors for the next glycosylation step without any further modifications. 


\section{Scheme 5. Synthesis of Saccharides 14 and 15}

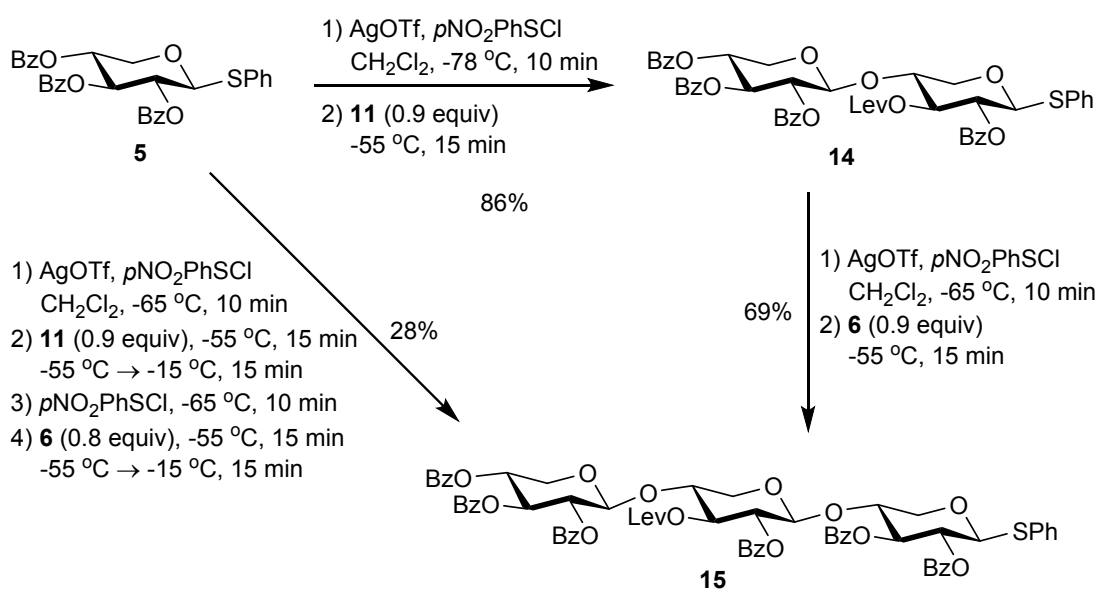

Accordingly, to prepare arabinoxylan 1 disaccharide 14 was then coupled twice with Levprotected acceptor 11 to afford trisaccharide $\mathbf{1 6}$ and tetrasaccharide $\mathbf{1 7}$ in 88 and 73\% yields, respectively (Scheme 6). The three Lev groups in the 3 positions were removed with hydrazine to give triol 18 in $96 \%$ yield. Subsequent glycosylation with four equiv. of imidate donor 4 afforded protected arabinoxylan 19 in $90 \%$ yield. The arabinofuranose donor $\mathbf{4}$ was prepared in four straightforward steps from the parent pentose. ${ }^{35,19 b}$ Thus, heptasaccharide $\mathbf{1 9}$ has been assembled from monosaccharides 4, 5 and $\mathbf{1 1}$ in a total of five steps and 48\% overall yield, which illustrates the advantage of the iterative pre-activation-based strategy.

\section{Scheme 6. Synthesis of Heptasaccharide 19}




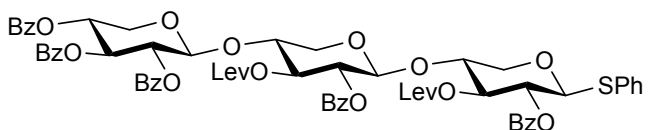

$88 \%$

16

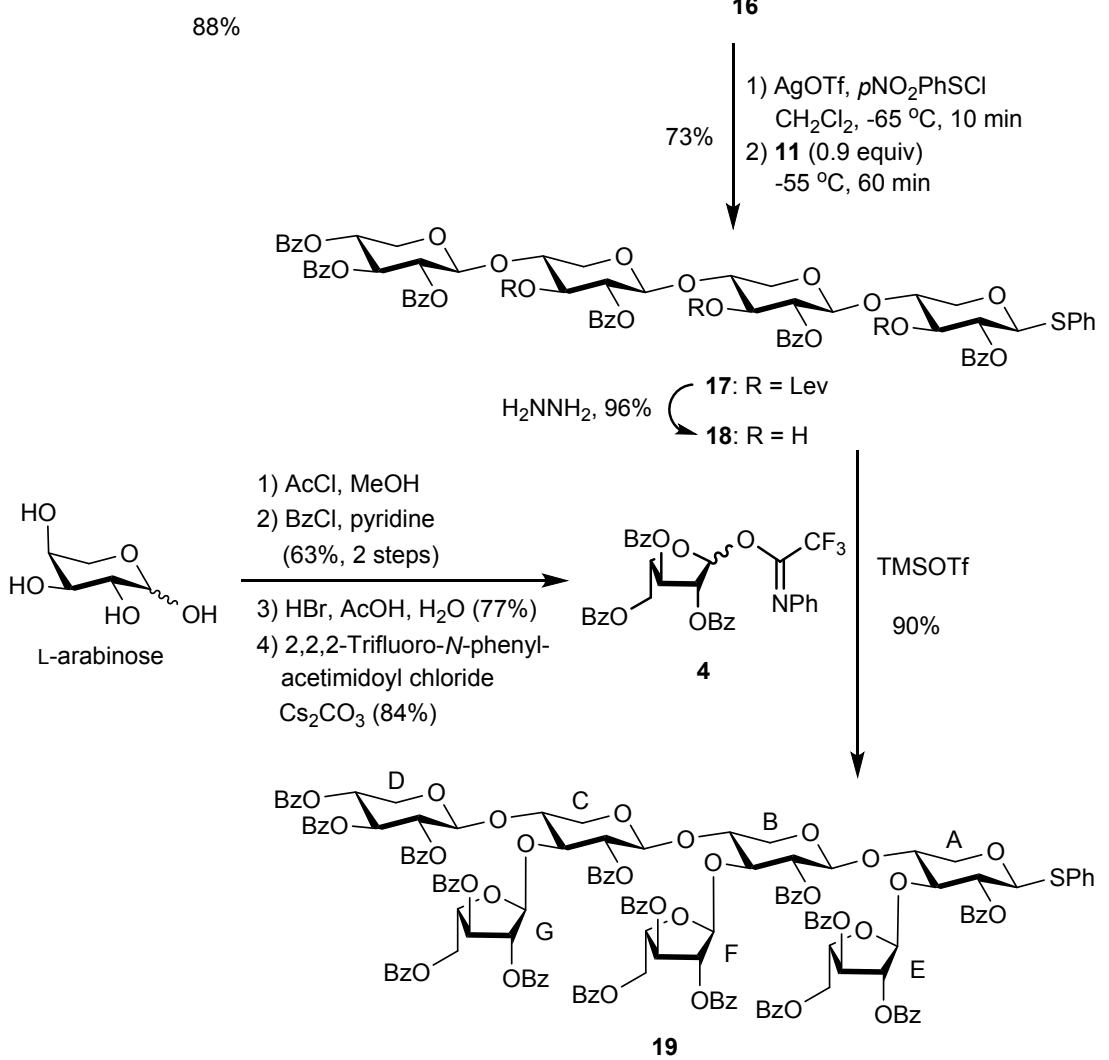

The same sequence of reactions were then employed to prepare the protected forms of arabinoxylans $\mathbf{2}$ and $\mathbf{3}$. To assemble the former, disaccharide $\mathbf{1 4}$ was used again and now coupled with 2,3-Lev-protected acceptor 9 to give trisaccharide $\mathbf{2 0}$ in 71\% yield (Scheme 7). Further glycosylation with acceptor 6 produced $61 \%$ yield of tetrasaccharide 21, which was subjected to Lev deprotection to afford triol 22. The arabinoses were then installed with donor 4 to afford heptasaccharide $\mathbf{2 3}$ in 95\% yield. Likewise, the route to arabinoxylan $\mathbf{3}$ began with the coupling between donor 5 and 2,3-Lev-protected acceptor 9 to furnish disaccharide 24 in $95 \%$ yield (Scheme 8). The following glycosylation with acceptor 11 produced trisaccharide 25 in $52 \%$ yield. The moderate yield in this coupling as well as between $\mathbf{2 0}$ and $\mathbf{6}$ in Scheme 7 may 
indicate a lower stability of the activated species with a Lev group at the 2 position. Next, trisaccharide 25 was reacted with acceptor $\mathbf{6}$ to give tetrasaccharide $\mathbf{2 6}$ in $75 \%$ yield, which was followed by Lev deprotection to provide triol 27 . The final triple glycosylation with arabinose donor $\mathbf{4}$ gave heptasaccharide $\mathbf{2 8}$ in $91 \%$ yield. Thus, protected heptasaccharides $\mathbf{2 3}$ and $\mathbf{2 8}$ have each been prepared in five steps from monosaccharides 4, 5,9 and 11 and with overall yields of 34 and 33\%, respectively. In all cases, the three arabinoses were installed in very high yields with imidate donor $\mathbf{4}$ proving the strength of this method even for forming densely substituted substrates.

\section{Scheme 7. Synthesis of Heptasaccharide 23}

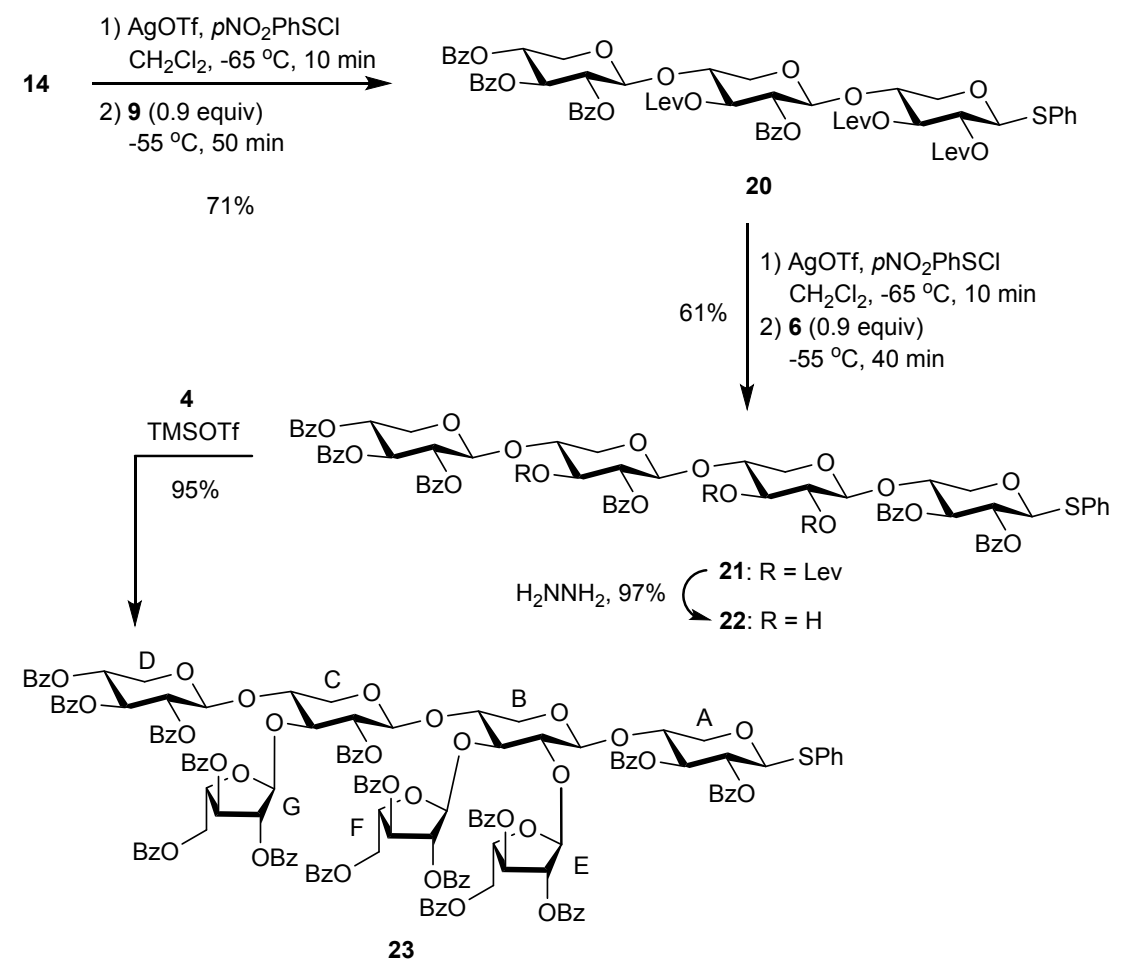

The $\beta$-linkages of the xylose backbones were confirmed by measuring the $\mathrm{J}_{\mathrm{CH}}$ coupling constants for the anomeric carbon atoms, which were found to be between 155 and $165 \mathrm{~Hz}$ 
where a coupling around $160 \mathrm{~Hz}$ is characteristic of a $\beta$-glycosidic bond. ${ }^{36}$ The anomeric carbon atoms for the $\beta$-glycosidic linkages were located in the $\delta=99.0-102.5 \mathrm{ppm}$ range, which is in accordance with earlier observations. ${ }^{11}$ Xylopyranosides are more conformationally flexible than e.g. glucopyranosides ${ }^{21}$ and the $\mathrm{J}_{\mathrm{H}-1, \mathrm{H}-2}$ coupling constants can therefore not always be used to determine their anomeric configuration. On the contrary, for the arabinofuranose substituents the $\mathrm{J}_{\mathrm{H}-1, \mathrm{H}-2}$ coupling constants could be used to verify the $\alpha$-linkages since small values of $0-2$ $\mathrm{Hz}$ are characteristic for the $\alpha$ anomers. ${ }^{37}$

\section{Scheme 8. Synthesis of Heptasaccharide 28}

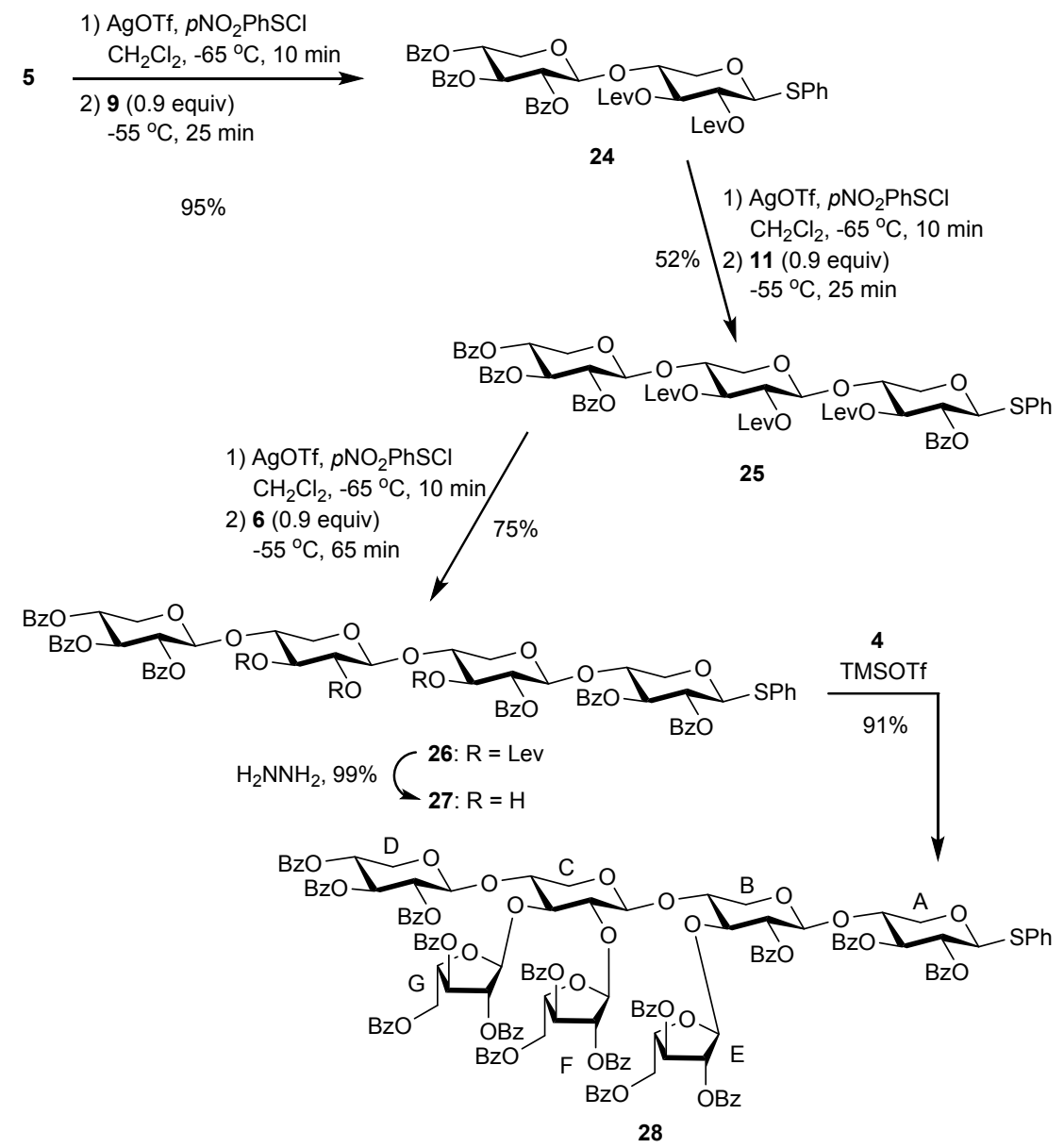


Deprotection of the three heptasaccharides is a two-step process since the thiophenyl moiety and the benzoates are removed under different conditions. In the first approach, the thiophenyl group was cleaved in the first step with NBS in a mixture of acetone and water. ${ }^{38}$ This transformation proceeded uneventfully for all three heptasaccharides and gave rise to reducing sugars $29-31$ in $92-93 \%$ yield (Scheme 9 ). The products were isolated as $\alpha / \beta$ mixtures with the $\alpha$ anomers as the major components according to NMR. The following Zemplén reaction to remove the benzoates ${ }^{39}$ was carried out with approx. 20 equiv. of $\mathrm{NaOMe}$ in a 1:1 methanoldichloromethane solution for up to 7 days to achieve complete deprotection of all benzoyl groups. Unfortunately, a mixture of saccharides of different length were obtained and it became apparent that the alkaline deprotection procedure had been accompanied by a so-called peeling reaction (endwise degradation). ${ }^{40}$ In this process reducing saccharides are degraded with one monosaccharide at the time from the reducing end through aldose - ketose isomerizations and $\beta$ alkoxy eliminations. ${ }^{40}$ To circumvent this side reaction, the order of the deprotections were reversed. First, the protected heptasaccharides were subjected to cleavage of the benzoyl groups, which proceeded cleanly to give compounds $32-34$ in $77-91 \%$ yield. Then, attempts were made to remove the thiophenyl moiety under the same conditions as applied to the perbenzoylated substrates. However, these conditions now resulted in partial hydrolysis of the arabinofuranose residues, which is presumably due to the higher acid lability of these substituents in the absence of the benzoyl groups. Hence, 2,6-lutidine was added during the deprotections $^{41}$ to ensure a $\mathrm{pH}$ of the reaction media around 6 , which led to clean removal of the thiophenyl groups to afford the completely deprotected target structures $\mathbf{1}-\mathbf{3}$ in $84-97 \%$ yield. Heptasaccharides $\mathbf{1}$ - $\mathbf{3}$ were fully characterized by $800 \mathrm{MHz}$ NMR spectroscopy and although they were obtained as $\alpha / \beta$ mixtures, the differences in the ${ }^{1} \mathrm{H}$ and the ${ }^{13} \mathrm{C}$ chemical shifs between 
the $\alpha$ and the $\beta$ anomers were only observed for the reducing end xylose unit and when an arabinose residue was attached to this moiety (as in $\mathbf{1}$ ).

\section{Scheme 9. Deprotection of Heptasaccharides 19, 23 and 28}

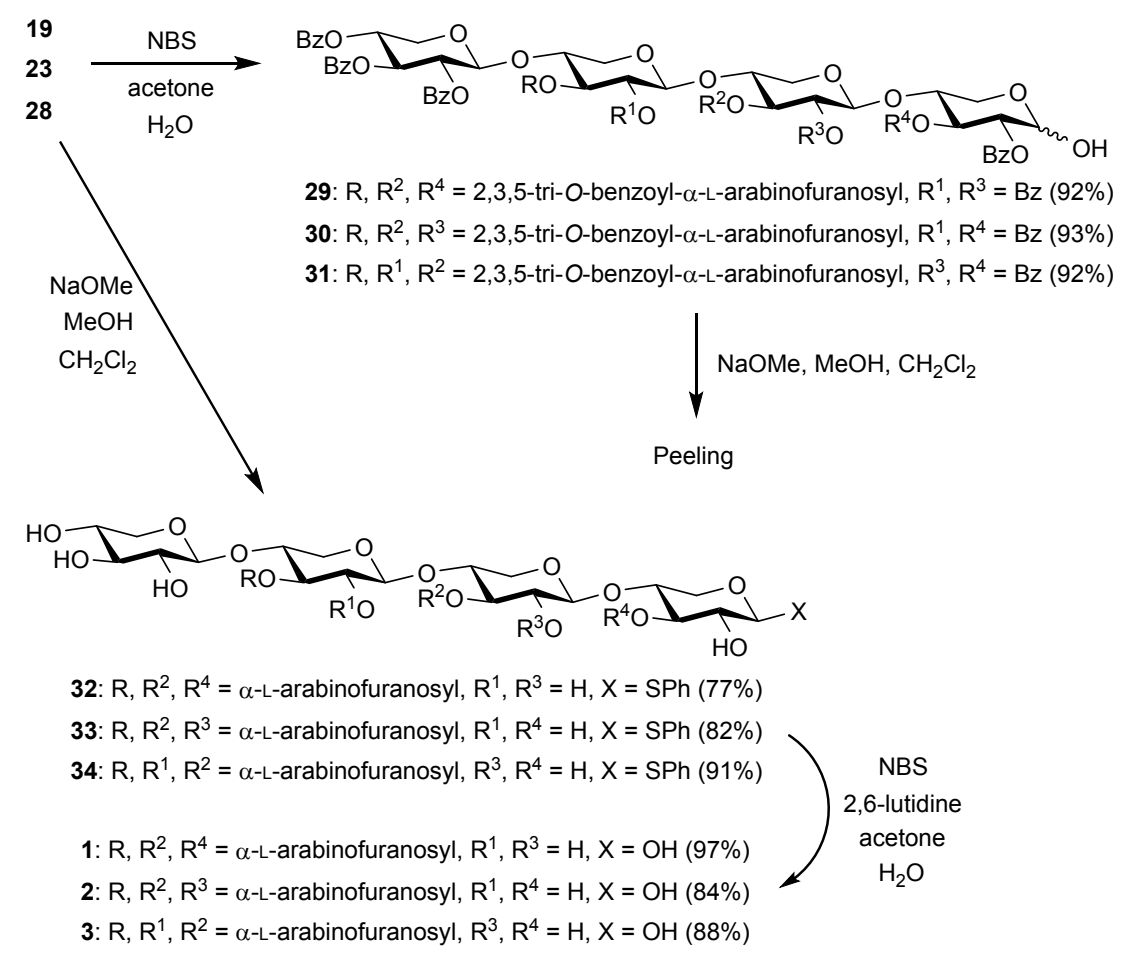

In conclusion, three arabinoxylan heptasaccharides have been assembled in seven steps from monosaccharide building blocks in overall yields of $36(\mathbf{1}), 24$ (2) and 27\% (3). The key transformations are the pre-activation-based thioglycoside glycosylation to prepare the tetraxylan backbones and the triple glycosylation with imidate donor $\mathbf{4}$ to install the arabinose substituents. $p$-Nitrobenzenesulfenyl chloride was employed as a potent and convenient source of the sulfenium electrophile for the pre-activation of the phenyl thioglycoside donors. In the ensuing glycosylation reaction, temperature monitoring revealed an optimum temperature for the coupling where side reactions are diminished. The strategy allows for the expeditious 
preparation of a diverse set of densely substituted arabinoxylans in good overall yields as valuable substrates for characterization of hemicellulose-degrading enzymes.

\section{EXPERIMENTAL SECTION}

General Information. Starting materials, reagents and solvents were purchased from commercial suppliers and used without further purification unless otherwise noted. All solvents were of analytical HPLC grade. Anhyd solvents were obtained from an Innovative Technology PS-MD-7 PureSolv solvent purification system except for $\mathrm{CH}_{2} \mathrm{Cl}_{2}$ and toluene for glycosylations, which were dried over $3 \AA$ mol sieves prior to use. All reactions were performed under inert atm $\left(\mathrm{N}_{2}\right)$ in oven-dried glassware. TLC was carried out using Merck aluminium sheets pre-coated with $0.25 \mathrm{~mm}$ silica gel, C-60 $\mathrm{F}_{254}$ plates. TLC plates were inspected under UV light or visualized by charring after dipping in a cerium ammonium sulfate solution (1\% cerium(IV)sulfate and 2.5\% ammonium heptamolybdate in a 10\% sulfuric acid solution). Flash column chromatography was performed using Merck Geduran silica gel $60 \AA$ (40-63 $\mu \mathrm{m})$ while dry column chromatography ${ }^{42}$ was accomplished using Merck silica gel $60 \AA$ (15-40 $\left.\mu \mathrm{m}\right)$. NMR spectra were recorded with a Bruker Ascend ${ }^{\mathrm{TM}} 400$ or a Bruker Ascend ${ }^{\mathrm{TM}} 800$ spectrometer. The chemical shift are reported in ppm relative to the residual solvent peak from $\mathrm{CDCl}_{3}\left(\delta_{\mathrm{H}}=7.26 \mathrm{ppm}, \delta_{\mathrm{C}}=77.16 \mathrm{ppm}\right), \mathrm{CD}_{3} \mathrm{OD}\left(\delta_{\mathrm{H}}=4.87 \mathrm{ppm}, \delta_{\mathrm{C}}=49.00 \mathrm{ppm}\right)$ or $\mathrm{D}_{2} \mathrm{O}\left(\delta_{\mathrm{H}}\right.$ $=4.79 \mathrm{ppm})$. Assignment of ${ }^{1} \mathrm{H}$ and ${ }^{13} \mathrm{C}$ resonances were based on APT, DQF-COSY, HSQC, H2BC, HMBC, TOCSY and HSQC-TOSCY experiments. Xylose residues are numbered A - D from the reducing end while arabinose substituents are labelled $\mathrm{E}-\mathrm{G}$ as shown in Scheme 6 8. Optical rotation was measured on a Perkin Elmer Model 341 polarimeter. HRMS analysis 
was performed on either a UHPLC-QTOF system (Dionex UltiMate 3000 and Bruker MaXis) with an electrospray ionization (ESI) source or a MALDI-TOF system (Bruker Solarix XR 7T).

Phenyl 1-thio- $\beta$-D-xylopyranoside (7). D-Xylose (29.9 g, 199 mmol), $\mathrm{Et}_{3} \mathrm{~N}$ (223 mL, $\left.160 \mathrm{mmol}\right)$ and DMAP (4.90 g, $40.1 \mathrm{mmol})$ were suspended in $\mathrm{CH}_{2} \mathrm{Cl}_{2}(300 \mathrm{~mL})$ at $0{ }^{\circ} \mathrm{C}$. Upon dropwise addition of $\mathrm{Ac}_{2} \mathrm{O}(94.0 \mathrm{~mL}, 996 \mathrm{mmol})$ the solution turned yellow. After stirring for $2 \mathrm{~h}$ at $\mathrm{rt}$ TLC analysis (hexane/EtOAc 1:1) showed the consumption of the starting material and formation of the product. The mixture was washed with $1 \mathrm{M} \mathrm{HCl}$ and brine followed by concentration in vacuo. The crude xylose tetraacetate was dissolved in dry $\mathrm{CH}_{2} \mathrm{Cl}_{2}(160 \mathrm{~mL})$ followed by addition of $\mathrm{PhSH}(25.0 \mathrm{~mL}, 243 \mathrm{mmol})$ and $\mathrm{BF}_{3} \cdot \mathrm{OEt}_{2}(76.0 \mathrm{~mL}, 600 \mathrm{mmol})$ at $0{ }^{\circ} \mathrm{C}$ under $\mathrm{N}_{2}$ atm. The reaction was stirred overnight at $\mathrm{rt}$ where it turned purple. TLC analysis (hexane/EtOAc 2:1) showed full consumption of the starting material and formation of the product. The solution was diluted with $\mathrm{CH}_{2} \mathrm{Cl}_{2}$ and washed with saturated aq $\mathrm{NaHCO}_{3}$ and brine, dried over $\mathrm{Na}_{2} \mathrm{SO}_{4}$, filtered and concd in vacuo. The residue was dissolved in methanol $(160 \mathrm{~mL})$ and solid sodium was added until a basic medium was obtained. After $1 \mathrm{~h}$ TLC analysis (hexane/EtOAc 2:1) showed the consumption of the starting material and formation of the product. The solution was neutralized with Amberlite IR-120 $\left(\mathrm{H}^{+}\right)$, filtered and concd in vacuo. The residue was purified by dry column chromatography ( $10 \%$ acetone in toluene, $5 \%$ gradient) followed by crystallization from hexane and acetone to afford a white crystalline product (19.2 g, 40\%). $R_{\mathrm{f}} 0.27$ (toluene/acetone 1:1). Mp 142.3-143.8 ${ }^{\circ} \mathrm{C}\left(\right.$ lit. $\left.{ }^{20} 143-145{ }^{\circ} \mathrm{C}\right) .{ }^{1} \mathrm{H}$ NMR (400 MHz, $\left.\mathrm{CD}_{3} \mathrm{OD}\right) \delta 7.54-7.51(\mathrm{~m}, 2 \mathrm{H}), 7.34-7.26(\mathrm{~m}, 3 \mathrm{H}), 4.56\left(\mathrm{~d}, J_{1,2}=9.3 \mathrm{~Hz}, 1 \mathrm{H}\right.$, H1), $3.95\left(\mathrm{dd}, J_{5,5},=11.3 \mathrm{~Hz}, J_{4,5}=5.2 \mathrm{~Hz}, 1 \mathrm{H}, \mathrm{H} 5\right), 3.51-3.45(\mathrm{~m}, 1 \mathrm{H}, \mathrm{H} 4), 3.38-3.31(\mathrm{~m}, 3 \mathrm{H}$, $\mathrm{H} 3, \mathrm{OH}), 3.26-3.19\left(\mathrm{~m}, 2 \mathrm{H}, \mathrm{H} 2, \mathrm{H} 5^{\prime}\right) .{ }^{13} \mathrm{C}\left\{{ }^{1} \mathrm{H}\right\}$ NMR (101 MHz, CD $\left.3 \mathrm{OD}\right) \delta 134.9,133.1(\times 2)$, 
$129.9(\times 2), 128.5,90.1(\mathrm{C} 1), 79.2(\mathrm{C} 3), 73.7(\mathrm{C} 2), 70.9(\mathrm{C} 4), 70.4(\mathrm{C} 5)$. NMR data are in accordance with literature values. ${ }^{43}$

Phenyl 2,3,4-tri-O-benzoyl-1-thio- $\beta$-D-xylopyranoside (5). Triol 7 (5.12 g, $21.1 \mathrm{mmol}$ ) was dissolved in pyridine $(45 \mathrm{~mL})$ and $\mathrm{BzCl}(7.4 \mathrm{~mL}, 63.4 \mathrm{mmol})$ was added. The reaction was stirred at $\mathrm{rt}$ for $1 \mathrm{~h}$ and excess of $\mathrm{BzCl}$ was quenched by the addition of methanol $(10 \mathrm{~mL})$ followed by stirring for an additional $10 \mathrm{~min}$. The mixture was diluted with $\mathrm{CH}_{2} \mathrm{Cl}_{2}$ and washed with $1 \mathrm{M} \mathrm{HCl}$ and water, dried over $\mathrm{Na}_{2} \mathrm{SO}_{4}$, filtered, and concd in vacuo. The residue was purified by flash column chromatography to afford 5 as write crystals $(5.1 \mathrm{~g}, 84 \%) . R_{\mathrm{f}} 0.28$ (heptane/EtOAc 7:3). Mp 102.9-104.3 ${ }^{\circ} \mathrm{C}(\mathrm{EtOH}) .{ }^{1} \mathrm{H}$ NMR $\left(400 \mathrm{MHz}, \mathrm{CDCl}_{3}\right) \delta 8.05(\mathrm{dd}, J=$ 8.2, 1.1 Hz, 2H), 8.01-7.98 (m, 4H), 7.56-7.51 (m, 5H), 7.42-7.32 (m, 9H), 5.78 (t, $J=6.6 \mathrm{~Hz}$, $1 \mathrm{H}, \mathrm{H} 3), 5.46(\mathrm{t}, J=6.3 \mathrm{~Hz}, 1 \mathrm{H}, \mathrm{H} 2), 5.33-5.26(\mathrm{~m}, 2 \mathrm{H}, \mathrm{H} 1, \mathrm{H} 4), 4.71\left(\mathrm{dd}, J_{5 \mathrm{eq}, 5 \mathrm{ax}}=12.3 \mathrm{~Hz}\right.$, $\left.J_{5 \mathrm{eq}, 4}=4.0 \mathrm{~Hz}, 1 \mathrm{H}, \mathrm{H} 5_{\mathrm{eq}}\right), 3.83\left(\mathrm{dd}, J_{5 \mathrm{ax}, 5 \mathrm{eq}}=12.3 \mathrm{~Hz}, J_{5 \mathrm{ax}, 4}=6.5 \mathrm{~Hz}, 1 \mathrm{H}, \mathrm{H} 5_{\mathrm{ax}}\right) \cdot{ }^{13} \mathrm{C}\left\{{ }^{1} \mathrm{H}\right\} \mathrm{NMR}$ $\left(101 \mathrm{MHz}, \mathrm{CDCl}_{3}\right) \delta 165.6,165.3,165.3,133.6,133.5,133.5,133.2,132.8(\times 2), 130.2(\times 2)$, $130.1(\times 2), 130.1(\times 2), 129.3(\times 2), 129.2(\times 2), 129.1,128.6(\times 2), 128.6(\times 2), 128.5(\times 2), 128.3$, 86.5 (C1), 70.6 (C3), 70.1 (C2), 68.8 (C4), 63.7 (C5). Anal. calcd for $\mathrm{C}_{32} \mathrm{H}_{26} \mathrm{O}_{7} \mathrm{~S}: \mathrm{C}, 69.30 ; \mathrm{H}$, 4.73; S, 5.78. Found: C, 69.26; H, 4.70; S, 5.66. NMR data are in accordance with literature values. ${ }^{44}$

Phenyl 4-O-(p-methoxy)benzyl-1-thio- $\beta$-D-xylopyranoside (8). To a solution of triol 7 (29.5 g, $122 \mathrm{mmol})$ in dry DMF $(200 \mathrm{~mL})$ was added CSA $(2.83 \mathrm{~g}, 12.0 \mathrm{mmol})$ and the mixture was heated to $60{ }^{\circ} \mathrm{C}$. 2-Methoxy prop-1-ene $(37.5 \mathrm{~mL}, 366 \mathrm{mmol})$ was added slowly to the solution. The reaction was stirred for $1 \mathrm{~h}$ and then cooled to $\mathrm{rt}$ and quenched with $\mathrm{Et}_{3} \mathrm{~N}(30 \mathrm{~mL})$. 
Concentration in vacuo followed by purification of the residue by flash column chromatography (heptane/EtOAc/ $\mathrm{CH}_{2} \mathrm{Cl}_{2}$ 4:1:1) afforded phenyl 2,3-O-isopropylidene-1-thio- $\beta$-Dxylopyranoside as a colorless oil $(24.1 \mathrm{~g}, 70 \%) . R_{\mathrm{f}} 0.62$ (toluene/acetone $\left.3: 1\right) .{ }^{1} \mathrm{H}$ NMR (400 $\left.\mathrm{MHz} \mathrm{CDCl}_{3}\right) \delta 7.58-7.55(\mathrm{~m}, 2 \mathrm{H}), 7.34-7.30(\mathrm{~m}, 3 \mathrm{H}), 4.80\left(\mathrm{~d}, J_{1,2}=9.6 \mathrm{~Hz}, 1 \mathrm{H}, \mathrm{H} 1\right), 4.13(\mathrm{dd}$, $\left.J_{5,5^{\prime}}=11.6 \mathrm{~Hz}, J_{4,5}=5.3 \mathrm{~Hz}, 1 \mathrm{H}, \mathrm{H} 5_{\mathrm{eq}}\right), 4.03-3.96(\mathrm{~m}, 1 \mathrm{H}, \mathrm{H} 4), 3.54(\mathrm{t}, J=9.1 \mathrm{~Hz}, 1 \mathrm{H}, \mathrm{H} 3)$, 3.27-3.22 (m, 2H, H2, H5 $\left.{ }_{\mathrm{ax}}\right), 2.17\left(\mathrm{~d}, J_{\mathrm{OH}, 4}=4.0 \mathrm{~Hz}, 1 \mathrm{H}, \mathrm{OH}\right), 1.49(\mathrm{~s}, 3 \mathrm{H}), 1.45(\mathrm{~s}, 3 \mathrm{H})$. ${ }^{13} \mathrm{C}\left\{{ }^{1} \mathrm{H}\right\}$ NMR $\left(101 \mathrm{MHz}, \mathrm{CDCl}_{3}\right) \delta 133.0(\times 2), 132.0,129.0(\times 2), 128.3,111.5,85.6(\mathrm{C} 1), 83.0$ (C3), 75.3 (C2), 70.0 (C5), 69.2 (C4), 26.8, 26.7. NMR data are in accordance with literature values. ${ }^{45}$ The intermediate $(17.5 \mathrm{~g}, 62.0 \mathrm{mmol})$ was dissolved in dry DMF $(120 \mathrm{~mL})$ and NaH (3.0 g, $74.4 \mathrm{mmol}, 60 \%$ in mineral oil) was added at $0{ }^{\circ} \mathrm{C}$. After $10 \mathrm{~min} \mathrm{PMBCl}(10.9 \mathrm{~mL}, 80.6$ mmol, 1.3 equiv.) was added. The mixture was stirred at $\mathrm{rt}$ for $16 \mathrm{~h}$ and then quenched with $10 \%$ $\mathrm{HCl}(28 \mathrm{~mL})$ and diluted with $\mathrm{CH}_{2} \mathrm{Cl}_{2}$. The solution was washed with saturated aq $\mathrm{NaHCO}_{3}$, dried over $\mathrm{Na}_{2} \mathrm{SO}_{4}$, filtered, and concd in vacuo. The crude residue was dissolved in $\mathrm{CH}_{2} \mathrm{Cl}_{2} / \mathrm{MeOH}$ 1:1 (200 mL), CSA (14.4 g, $\left.62.0 \mathrm{mmol}\right)$ was added and the mixture was allowed to stir at rt overnight. The reaction was quenched by addition of $\mathrm{Et}_{3} \mathrm{~N}$ and concd in vacuo. The residue was purified by flash column chromatography (heptane/EtOAc 3:2) to furnish compound $\mathbf{8}$ as a white crystalline solid (20.5 g, 91\% over 2 steps). $R_{\mathrm{f}} 0.47$ (hexane/EtOAc 1:1). $\mathrm{Mp} 72.7-75.6{ }^{\circ} \mathrm{C}(\mathrm{EtOH}) .[\alpha]^{25} \mathrm{D}-55.9\left(\mathrm{c} 1, \mathrm{CHCl}_{3}\right) .{ }^{1} \mathrm{H} \mathrm{NMR}\left(400 \mathrm{MHz}, \mathrm{CDCl}_{3}\right) \delta 7.53-7.50$ $(\mathrm{m}, 2 \mathrm{H}), 7.33-7.29(\mathrm{~m}, 3 \mathrm{H}), 7.27-7.23(\mathrm{~m}, 2 \mathrm{H}), 6.90-6.86(\mathrm{~m}, 2 \mathrm{H}), 4.61\left(\mathrm{~d}, J_{\mathrm{gem}}=11.4 \mathrm{~Hz}, 1 \mathrm{H}\right.$, PMB), 4.58-4.54 (m, 2H, H1 ( $\left.\left.J_{1,2}=8.9 \mathrm{~Hz}\right) \mathrm{PMB}\right), 4.07\left(\mathrm{dd}, J_{5 \mathrm{eq}, 5 \mathrm{ax}}=11.5 \mathrm{~Hz}, J_{5 \mathrm{eq}, 4}=4.8 \mathrm{~Hz}\right.$, $\left.1 \mathrm{H}, \mathrm{H} 5_{\mathrm{eq}}\right), 3.80(\mathrm{~s}, 3 \mathrm{H}, \mathrm{OMe}), 3.66(\mathrm{t}, J=8.4 \mathrm{~Hz}, 1 \mathrm{H}, \mathrm{H} 3), 3.49-3.43(\mathrm{~m}, 1 \mathrm{H}, \mathrm{H} 4), 3.40(\mathrm{t}, J=$ $8.6 \mathrm{~Hz}, 1 \mathrm{H}, \mathrm{H} 2), 3.27\left(\mathrm{dd}, J_{5 \mathrm{ax}, 5 \mathrm{eq}}=11.5 \mathrm{~Hz}, J_{5 \mathrm{ax}, 4}=9.7 \mathrm{~Hz}, 1 \mathrm{H}, \mathrm{H} 5_{\mathrm{ax}}\right), 2.52(\mathrm{bs}, 2 \mathrm{H}, \mathrm{OH})$. ${ }^{13} \mathrm{C}\left\{{ }^{1} \mathrm{H}\right\}$ NMR $\left(101 \mathrm{MHz}, \mathrm{CDCl}_{3}\right) \delta 159.7,132.8(\times 2), 132.3,130.1,129.7(\times 2), 129.2(\times 2)$, 
128.3, $114.2(\times 2), 88.8(\mathrm{C} 1), 76.6(\mathrm{C} 4), 76.5(\mathrm{C} 3), 72.8$ (PMB), 72.1 (C2), 67.1 (C5), 55.4

(OMe). HRMS: $m / z$ calcd for $\mathrm{C}_{19} \mathrm{H}_{22} \mathrm{O}_{5} \mathrm{SNa}[\mathrm{M}+\mathrm{Na}]^{+}$385.1080, found 385.1090.

Phenyl 2,3-di-O-benzoyl-1-thio- $\beta$-D-xylopyranoside (6). Compound 8 (1.97 g, 5.43 mmol) was dissolved in $\mathrm{CH}_{2} \mathrm{Cl}_{2}(32 \mathrm{~mL})$ and $\mathrm{Et}_{3} \mathrm{~N}$ (2.4 mL, $\left.17.2 \mathrm{mmol}\right)$, DMAP (0.069 g, $\left.0.56 \mathrm{mmol}\right)$ and $\mathrm{BzCl}(1.9 \mathrm{~mL}, 16.4 \mathrm{mmol})$ were added at $0^{\circ} \mathrm{C}$. After $5 \mathrm{~min}$ the reaction was left to stir at $\mathrm{rt}$ overnight. 3-(Dimethylamino)-1-propylamine (2.2 mL, $17.5 \mathrm{mmol})$ was added and the mixture was stirred for $1.5 \mathrm{~h}$ followed by washing with $1 \mathrm{M} \mathrm{HCl}$ and brine, drying over $\mathrm{MgSO}_{4}$, filtration, and concentration in vacuo. The product phenyl 2,3-di-O-benzoyl-4- $O$ - $(p$ methoxy)benzyl-1-thio- $\beta$-D-xylopyranoside (3.12 g, 100\%) was obtained as a light yellow syrup and used without further purification. $R_{\mathrm{f}} 0.28$ (hexane/EtOAc 4:1). $[\alpha]^{25}{ }_{\mathrm{D}}+62.0\left(c 1.0, \mathrm{CHCl}_{3}\right)$. ${ }^{1} \mathrm{H}$ NMR (400 MHz, $\left.\mathrm{CDCl}_{3}\right) \beta$ 7.99-7.95 (m, 3H), 7.56-7.27 (m, 12H), 7.15-7.12 (m, 2H), $6.74-6.71(\mathrm{~m}, 2 \mathrm{H}), 5.60(\mathrm{t}, J=8.0 \mathrm{~Hz}, 1 \mathrm{H}, \mathrm{H} 3), 5.34(\mathrm{t}, J=8.0 \mathrm{~Hz}, 1 \mathrm{H}, \mathrm{H} 2), 5.04\left(\mathrm{~d}, J_{1,2}=8.1\right.$ $\mathrm{Hz}, 1 \mathrm{H}, \mathrm{H1}), 4.56$ (d, $\left.J_{\text {gem }}=11.9 \mathrm{~Hz}, 1 \mathrm{H}, \mathrm{PMB}\right), 4.52$ (d, $\left.J_{\text {gem }}=11.8 \mathrm{~Hz}, 1 \mathrm{H}, \mathrm{PMB}\right), 4.28$ (dd, $\left.J_{5 \mathrm{eq}, 5 \mathrm{ax}}=11.9 \mathrm{~Hz}, J_{5 \mathrm{eq}, 4}=4.6 \mathrm{~Hz}, 1 \mathrm{H}, \mathrm{H} 5_{\mathrm{eq}}\right), 3.80-3.76(\mathrm{~m}, 1 \mathrm{H}, \mathrm{H} 4), 3.75(\mathrm{~s}, 3 \mathrm{H}, \mathrm{OMe}), 3.57$ $\left(\mathrm{dd}, J_{5 \mathrm{ax}, 5 \mathrm{eq}}=11.9 \mathrm{~Hz}, J_{5 \mathrm{ax}, 4}=8.5 \mathrm{~Hz}, 1 \mathrm{H}, \mathrm{H} 5_{\mathrm{ax}}\right) \cdot{ }^{13} \mathrm{C}\left\{{ }^{1} \mathrm{H}\right\} \mathrm{NMR}\left(101 \mathrm{MHz}, \mathrm{CDCl}_{3}\right) \delta 165.7$, $165.4,159.5,133.4(\times 2), 133.2,132.5(\times 2), 130.1(\times 2), 130.0(\times 2), 129.7,129.7(\times 2), 129.5$, $129.4,129.1(\times 2), 128.5(\times 2), 128.5(\times 2), 128.1,113.9(\times 2), 87.0(\mathrm{C} 1), 73.9(\mathrm{C} 4), 73.6(\mathrm{C} 3)$, 72.5 (PMB), 70.6 (C2), 66.4 (C5), 55.4 (OMe). HRMS: $m / z$ calcd for $\mathrm{C}_{33} \mathrm{H}_{30} \mathrm{O}_{7} \mathrm{SNa}[\mathrm{M}+\mathrm{Na}]^{+}$ 593.1604, found 593.1617. NMR data are in accordance with literature values. ${ }^{13}$ To a well stirred emulsion of the above PMB ether $(0.696 \mathrm{~g}, 1.22 \mathrm{mmol})$ in $\mathrm{CH}_{2} \mathrm{Cl}_{2}(10 \mathrm{~mL})$ and $\mathrm{H}_{2} \mathrm{O}(1.0$ $\mathrm{mL})$, DDQ (0.415 $\mathrm{g}, 1.83 \mathrm{mmol})$ was added. The reaction was stirred in the dark for $7 \mathrm{~h}$ and then filtered through a large pad of Celite. The filtrate was washed with saturated aq $\mathrm{NaHCO}_{3}$ 
and brine, filtered and concd in vacuo. Purification by flash column chromatography (pentane/EtOAc 4:1) yielded 6 as a white crystalline compound $(0.514 \mathrm{~g}, 94 \%) . R_{\mathrm{f}} 0.53$ (hexane/EtOAc 2:1). Mp 133.1-134.0 ${ }^{\circ} \mathrm{C}(\mathrm{EtOH}) .[\alpha]^{25}{ }_{\mathrm{D}}+66.1\left(c\right.$ 1.0, $\left.\mathrm{CHCl}_{3}\right) .{ }^{1} \mathrm{H} \mathrm{NMR}(400$ $\left.\mathrm{MHz}, \mathrm{CDCl}_{3}\right) \delta 8.05-7.99(\mathrm{~m}, 4 \mathrm{H}), 7.57-7.49(\mathrm{~m}, 4 \mathrm{H}), 7.43-7.39(\mathrm{~m}, 4 \mathrm{H}), 7.35-7.29(\mathrm{~m}, 3 \mathrm{H})$, $5.43(\mathrm{t}, J=7.4 \mathrm{~Hz}, 1 \mathrm{H}, \mathrm{H} 2), 5.33(\mathrm{t}, J=7.4 \mathrm{~Hz}, 1 \mathrm{H}, \mathrm{H} 3), 5.09$ (d, $\left.J_{1,2}=7.3 \mathrm{~Hz}, 1 \mathrm{H}, \mathrm{H} 1\right), 4.45$ $\left(\mathrm{dd}, J_{5 \mathrm{eq}, 5 \mathrm{ax}}=12.0 \mathrm{~Hz}, J_{5 \mathrm{eq}, 4}=4.4 \mathrm{~Hz}, 1 \mathrm{H}, \mathrm{H} 5_{\mathrm{eq}}\right), 4.00(\mathrm{q}, J=6.9 \mathrm{~Hz}, 1 \mathrm{H}, \mathrm{H} 4), 3.61\left(\mathrm{dd}, J_{5 \mathrm{ax}, 5 \mathrm{eq}}=\right.$ $\left.12.0 \mathrm{~Hz}, J_{5 \mathrm{ax}, 4}=7.9 \mathrm{~Hz}, 1 \mathrm{H}, \mathrm{H} 5_{\mathrm{ax}}\right), 3.03(\mathrm{~s}, 1 \mathrm{H}, \mathrm{OH}) .{ }^{13} \mathrm{C}\left\{{ }^{1} \mathrm{H}\right\} \mathrm{NMR}\left(101 \mathrm{MHz}, \mathrm{CDCl}_{3}\right) \delta 167.1$, $165.2,133.8,133.6,133.0,132.8(\times 2), 130.2(\times 2), 130.0(\times 2), 129.3,129.2(\times 2), 128.9,128.7$ (×2), $128.7(\times 2), 128.3,86.8(\mathrm{C} 1), 76.0(\mathrm{C} 3), 70.2(\mathrm{C} 2), 68.4(\mathrm{C} 4), 67.6(\mathrm{C} 5) . \mathrm{HRMS}: \mathrm{m} / z$ calcd for $\mathrm{C}_{25} \mathrm{H}_{22} \mathrm{O}_{6} \mathrm{SNa}[\mathrm{M}+\mathrm{Na}]^{+} 473.1029$, found 473.1048 .

Phenyl 2,3-di-O-levulinoyl-1-thio- $\beta$-D-xylopyranoside (9). A solution of diol 8 (1.98 g, 5.47 mmol) and 4-oxopentanoic acid $(1.61 \mathrm{~g}, 13.8 \mathrm{mmol})$ in $\mathrm{CH}_{2} \mathrm{Cl}_{2}(8.5 \mathrm{~mL})$ was cooled to $0{ }^{\circ} \mathrm{C}$. Then a solution of DMAP $(0.070 \mathrm{~g}, 0.58 \mathrm{mmol})$ and DDC (3.42 $\mathrm{g}, 16.8 \mathrm{mmol})$ in $\mathrm{CH}_{2} \mathrm{Cl}_{2}(1.0$ $\mathrm{mL}$ ) was added. The reaction stirred at $\mathrm{rt}$ overnight and then filtered and concd. The residue was dissolved in $\mathrm{CH}_{2} \mathrm{Cl}_{2}(20 \mathrm{~mL})$ and $\mathrm{H}_{2} \mathrm{O}(2.0 \mathrm{~mL})$ and stirred vigorously. DDQ (1.86 g, 8.21 mmol) was added and the mixture was stirred at $\mathrm{rt}$ for $4.5 \mathrm{~h}$. Then the solution was filtered through a large pad of Celite and washed with saturated aq $\mathrm{NaHCO}_{3}$ and brine, dried over $\mathrm{Na}_{2} \mathrm{SO}_{4}$, filtered, and concd in vacuo. The residue was purified by flash column chromatography (hexane/EtOAc 1:1) to provide 9 as a light yellow wax (1.78 g, 74\% over 2 steps). $R_{\mathrm{f}} 0.58$ (EtOAc). $[\alpha]^{25} \mathrm{D}-23.4\left(c\right.$ 1.0, $\left.\mathrm{CHCl}_{3}\right) .{ }^{1} \mathrm{H} \mathrm{NMR}\left(400 \mathrm{MHz}, \mathrm{CDCl}_{3}\right) \delta 7.48-7.46(\mathrm{~m}, 2 \mathrm{H})$, $7.33-7.29(\mathrm{~m}, 3 \mathrm{H}), 5.02(\mathrm{t}, J=8.4 \mathrm{~Hz}, 1 \mathrm{H}, \mathrm{H} 3), 4.94(\mathrm{t}, J=8.7 \mathrm{~Hz}, 1 \mathrm{H}, \mathrm{H} 2), 4.73\left(\mathrm{~d}, J_{1,2}=8.8\right.$ $\mathrm{Hz}, 1 \mathrm{H}, \mathrm{H1}), 4.19\left(\mathrm{dd}, J_{5 \mathrm{eq}, 5 \mathrm{ax}}=11.7 \mathrm{~Hz}, J_{5 \mathrm{eq}, 4}=5.1 \mathrm{~Hz}, 1 \mathrm{H}, \mathrm{H} 5_{\mathrm{eq}}\right), 3.84-3.79(\mathrm{~m}, 1 \mathrm{H}, \mathrm{H} 4), 3.39$ 
$\left(\mathrm{dd}, J_{5 \mathrm{ax}, 5 \mathrm{eq}}=11.7 \mathrm{~Hz}, J_{5 \mathrm{ax}, 4}=9.5 \mathrm{~Hz}, 1 \mathrm{H}, \mathrm{H} 5_{\mathrm{ax}}\right), 3.16(\mathrm{bs}, 1 \mathrm{H}, \mathrm{OH}), 2.89-2.51(\mathrm{~m}, 8 \mathrm{H}), 2.17(2$ $\times \mathrm{s}, 6 \mathrm{H}) .{ }^{13} \mathrm{C}\left\{{ }^{1} \mathrm{H}\right\} \mathrm{NMR}\left(101 \mathrm{MHz}, \mathrm{CDCl}_{3}\right) \delta 208.0,206.4,173.0,171.4,132.7,132.6(\times 2)$, $129.1(\times 2), 128.2,86.8(\mathrm{C} 1), 76.5(\mathrm{C} 3), 69.7$ (C2), $68.6(\mathrm{C} 5), 68.5(\mathrm{C} 4), 38.4,37.9,29.9,29.9$, 28.3, 28.1. HRMS: $m / z$ calcd for $\mathrm{C}_{21} \mathrm{H}_{26} \mathrm{O}_{8} \mathrm{SNa}[\mathrm{M}+\mathrm{Na}]^{+} 461.1241$, found 461.1242.

Phenyl 2-O-benzoyl-4-O-(p-methoxy)benzyl-1-thio- $\beta$-D-xylopyranoside (10). To a vigorously stirred solution of diol $8(1.0 \mathrm{~g}, 2.76 \mathrm{mmol})$, tetrabutylammonium hydrogen sulfate $(0.187 \mathrm{~g}$, $0.55 \mathrm{mmol})$ and $\mathrm{BzCl}(0.43 \mathrm{~mL}, 3.72 \mathrm{mmol})$ in $\mathrm{CH}_{2} \mathrm{Cl}_{2}(50 \mathrm{~mL})$ at $-5^{\circ} \mathrm{C}$ was added a $1 \mathrm{M}$ aq $\mathrm{NaOH}$ solution $(7.0 \mathrm{~mL}, 7.0 \mathrm{mmol})$. The mixture was stirred for $30 \mathrm{~min}$ (TLC indicated traces of $\mathbf{8}$ and the dibenzoylated product). The organic layer was separated, washed with $\mathrm{H}_{2} \mathrm{O}$, dried over $\mathrm{Na}_{2} \mathrm{SO}_{4}$, filtered, and concd in vacuo. The residue was purified by flash column chromatography (hexane/EtOAc 8.5:1.5) affording compound $\mathbf{1 0}$ as white crystals $(0.877 \mathrm{~g}$, 68\%). $R_{\mathrm{f}} 0.35$ (hexane/EtOAc 3:1). Mp 101.7-102.4 ${ }^{\circ} \mathrm{C}(\mathrm{EtOH}) .[\alpha]^{25} \mathrm{D}-13.2\left(c 1.0, \mathrm{CHCl}_{3}\right)$. ${ }^{1} \mathrm{H}$ NMR $\left(400 \mathrm{MHz}, \mathrm{CDCl}_{3}\right) \delta 8.09-8.07(\mathrm{~m}, 2 \mathrm{H}), 7.61-7.57(\mathrm{~m}, 1 \mathrm{H}), 7.48-7.41(\mathrm{~m}, 4 \mathrm{H})$, 7.29-7.24 (m, 5H), 6.89-6.86 (m, 2H), $5.04\left(\mathrm{dd}, J_{1,2}=9.5 \mathrm{~Hz}, J_{2,3}=9.0 \mathrm{~Hz}, 1 \mathrm{H}, \mathrm{H} 2\right), 4.78(\mathrm{~d}$, $\left.J_{1,2}=9.6 \mathrm{~Hz}, 1 \mathrm{H}, \mathrm{H} 1\right), 4.65\left(\mathrm{~d}, J_{\mathrm{gem}}=11.5 \mathrm{~Hz}, 1 \mathrm{H}, \mathrm{PMB}\right), 4.60\left(\mathrm{~d}, J_{\mathrm{gem}}=11.5 \mathrm{~Hz}, 1 \mathrm{H}, \mathrm{PMB}\right)$, $4.10\left(\mathrm{dd}, J_{5 \mathrm{eq}, 5 \mathrm{ax}}=11.5 \mathrm{~Hz}, J_{5 \mathrm{eq}, 4}=5.1 \mathrm{~Hz}, 1 \mathrm{H}, \mathrm{H} 5_{\mathrm{eq}}\right), 3.85(\mathrm{t}, J=8.8 \mathrm{~Hz}, 1 \mathrm{H}, \mathrm{H} 3), 3.81(\mathrm{~s}, 3 \mathrm{H}$, OMe), 3.61-3.55 (m, 1H, H4), $3.30\left(\mathrm{dd}, J_{5 \mathrm{ax}, 5 \mathrm{eq}}=11.5 \mathrm{~Hz}, J_{5 \mathrm{ax}, 4}=10.1 \mathrm{~Hz}, 1 \mathrm{H}, \mathrm{H} 5_{\mathrm{ax}}\right), 2.62(\mathrm{bs}$, $1 \mathrm{H}, \mathrm{OH}) .{ }^{13} \mathrm{C}\left\{{ }^{1} \mathrm{H}\right\} \mathrm{NMR}\left(101 \mathrm{MHz}, \mathrm{CDCl}_{3}\right) \delta 166.1,159.7,133.5,132.8(\times 2), 132.7,130.1$ $(\times 2), 130.1,129.8,129.7(\times 2), 129.1(\times 2), 128.6(\times 2), 128.2,114.2(\times 2), 86.8(\mathrm{C} 1), 77.4(\mathrm{C} 4)$, 76.1 (C3), 73.1 (PMB), 73.0 (C2), 67.6 (C5), 55.4 (OMe). HRMS: $m / z$ calcd for $\mathrm{C}_{26} \mathrm{H}_{26} \mathrm{O}_{6} \mathrm{SNa}$ $[\mathrm{M}+\mathrm{Na}]^{+}$489.1342, found 489.1343. 
Phenyl 2-O-benzoyl-3-O-levulinoyl-1-thio- $\beta$-D-xylopyranoside (11). To a solution of $\mathbf{1 0}$ (3.94 g, $8.45 \mathrm{mmol})$ and 4-oxopentanoic acid $(1.22 \mathrm{~g}, 10.5 \mathrm{mmol})$ in $\mathrm{CH}_{2} \mathrm{Cl}_{2}(14 \mathrm{~mL})$ cooled to $0{ }^{\circ} \mathrm{C}$ was added DMAP (0.104 g, $0.85 \mathrm{mmol})$ and DCC (2.61 g, $12.6 \mathrm{mmol})$. The solution was left to stir at $\mathrm{rt}$ for $3.5 \mathrm{~h}$, then filtered and concd in vacuo. The residue was dissolved in $\mathrm{CH}_{2} \mathrm{Cl}_{2}(60 \mathrm{~mL})$ and $\mathrm{H}_{2} \mathrm{O}(6.0 \mathrm{~mL})$ and stirred vigorously. DDQ $(2.88 \mathrm{~g}, 12.7 \mathrm{mmol})$ was added and the mixture was left to stir at rt overnight in the dark. Then the solution was filtered through a large pad of Celite and washed with saturated aq $\mathrm{NaHCO}_{3}$ and brine, dried over $\mathrm{Na}_{2} \mathrm{SO}_{4}$, filtered, and concd in vacuo. The residue was purified by flash column chromatography (hexane/EtOAc 2:1) affording 11 as a white crystalline solid (2.935 g, 78\% over 2 steps). $R_{\mathrm{f}} 0.28$ (hexane/EtOAc 1:1). Mp 88.7-90.3 ${ }^{\circ} \mathrm{C}(\mathrm{EtOH}) .[\alpha]^{25} \mathrm{D}-1.8\left(c 1.0, \mathrm{CHCl}_{3}\right) .{ }^{1} \mathrm{H}$ NMR $\left(400 \mathrm{MHz}, \mathrm{CDCl}_{3}\right) \delta$ 8.03-8.01 (m, 2H), $7.59(\mathrm{t}, J=7.4 \mathrm{~Hz}, 1 \mathrm{H}), 7.47-7.44(\mathrm{~m}, 4 \mathrm{H}), 7.29-7.28(\mathrm{~m}, 3 \mathrm{H}), 5.23-5.16$ (m, 2H, H2, H3), 4.94-4.91 (m, 1H, H1), 4.29 (dd, $\left.J_{5 \text { eq, } 5 \mathrm{ax}}=11.8 \mathrm{~Hz}, J_{5 \mathrm{eq}, 4}=4.9 \mathrm{~Hz}, 1 \mathrm{H}, \mathrm{H} 5_{\text {eq }}\right)$, 3.94-3.89 (m, 1H, H4), 3.49 (dd, $\left.J_{5 \mathrm{ax}, 5 \mathrm{eq}}=11.7 \mathrm{~Hz}, J_{5 \mathrm{ax}, 4}=9.1 \mathrm{~Hz}, 1 \mathrm{H}, \mathrm{H} 5_{\mathrm{ax}}\right), 3.14(\mathrm{bs}, 1 \mathrm{H}$, $\mathrm{OH}), 2.81-2.65(\mathrm{~m}, 2 \mathrm{H}), 2.58-2.51(\mathrm{~m}, 1 \mathrm{H}), 2.44-2.37(\mathrm{~m}, 1 \mathrm{H}), 2.11(\mathrm{~s}, 3 \mathrm{H}) .{ }^{13} \mathrm{C}\left\{{ }^{1} \mathrm{H}\right\} \mathrm{NMR}$ $\left(101 \mathrm{MHz}, \mathrm{CDCl}_{3}\right) \delta 207.7,172.8,165.3,133.6,132.9,132.7(\times 2), 130.0(\times 2), 129.4,129.1$ (×2), $128.7(\times 2), 128.2,87.0(\mathrm{C} 1), 76.4(\mathrm{C} 3), 70.3(\mathrm{C} 2), 68.5(\mathrm{C} 4), 68.3$ (C5), 38.5, 29.8, 28.3. HRMS: $m / z$ calcd for $\mathrm{C}_{23} \mathrm{H}_{24} \mathrm{O}_{7} \mathrm{SNa}[\mathrm{M}+\mathrm{Na}]^{+} 467.1135$, found 467.1136.

Temperature Screening (General Procedure A). Crushed mol sieves ( $3 \AA, 1.0 \mathrm{~g}$ ) were added a 2-neck Schlenk flask, where the middle neck was fitted with a glass stopper and the other with a septum. The flask was placed under vacuum, heated with a heatgun and then subjected to an atm of $\mathrm{N}_{2}$ and cooled to rt. Donor $5(0.200 \mathrm{~g}, 0.36 \mathrm{mmol})$ dissolved in dry $\mathrm{CH}_{2} \mathrm{Cl}_{2}(3.0 \mathrm{~mL})$ was added to the flask together with AgOTf $(0.185 \mathrm{~g}, 0.72 \mathrm{mmol})$ dissolved in dry toluene $(2.0 \mathrm{~mL})$. 
Stirring of the reaction was initiated and the glass stopper was exchanged for a thermometer, and the solution was cooled to $-65^{\circ} \mathrm{C} . p \mathrm{NO}_{2} \mathrm{PhSCl}(0.068 \mathrm{~g}, 0.36 \mathrm{mmol})$ was dissolved in dry $\mathrm{CH}_{2} \mathrm{Cl}_{2}(0.5 \mathrm{~mL})$ and slowly added followed by stirring for an additional $10 \mathrm{~min}$. Acceptor 6 (0.146 g, $0.33 \mathrm{mmol})$ was dissolved in dry $\mathrm{CH}_{2} \mathrm{Cl}_{2}(0.5 \mathrm{~mL})$ and added quickly. The temperature was raised as indicated in Figure 1, where the reaction was allowed to warm to $-15^{\circ} \mathrm{C}$ at which point $\mathrm{Et}_{3} \mathrm{~N}(0.15 \mathrm{~mL}, 1.08 \mathrm{mmol})$ was added. The solution was filtered through a pad of Celite and concd in vacuo. The residue was purified by flash column chromatography to afford the product 12 .

Phenyl 2,3,4-tri-O-benzoyl- $\beta$-D-xylopyranosyl- $(1 \rightarrow 4)$-2,3-di-O-benzoyl-1-thio- $\beta$ - $D$ xylopyranoside (12). To a Schlenk flask containing $3 \AA$ mol sieves (1.20 g) was added donor 5 $(0.200 \mathrm{~g}, 0.36 \mathrm{mmol})$ dissolved in dry $\mathrm{CH}_{2} \mathrm{Cl}_{2}(6.0 \mathrm{~mL})$ and $\operatorname{AgOTf}(0.185 \mathrm{~g}, 0.72 \mathrm{mmol})$ dissolved in dry $\mathrm{Et}_{2} \mathrm{O}(5.0 \mathrm{~mL})$. The mixture was stirred for $1 \mathrm{~h}$ at $\mathrm{rt}$ and then cooled to $-78^{\circ} \mathrm{C}$ at which point $\mathrm{PhSCl}(0.062 \mathrm{~g}, 0.43 \mathrm{mmol})$ or $p \mathrm{NO}_{2} \mathrm{PhSCl}(0.082 \mathrm{~g}, 0.43 \mathrm{mmol})$ dissolved in dry $\mathrm{CH}_{2} \mathrm{Cl}_{2}(1.0 \mathrm{~mL})$ was added dropwise followed by stirring for $10 \mathrm{~min}$. Acceptor $6(0.146 \mathrm{~g}$, $0.33 \mathrm{mmol})$ dissolved in dry $\mathrm{CH}_{2} \mathrm{Cl}_{2}(0.5 \mathrm{~mL})$ was then added slowly. The reaction was stirred at $-78{ }^{\circ} \mathrm{C}$ for $15 \mathrm{~min}$, then for $15 \mathrm{~min}$ at $\mathrm{rt}$ after which $\mathrm{Et}_{3} \mathrm{~N}(0.21 \mathrm{~mL}, 2.88 \mathrm{mmol})$ was added. The mixture was filtered through a pad of Celite, concd in vacuo, and purified by flash column chromatography (pentane/EtOAc 3:2) to give 12 as a white amorphous solid (0.186 g, 64\% with $\mathrm{PhSCl}$ and $0.229 \mathrm{~g}, 79 \%$ with $\left.\mathrm{pNO}_{2} \mathrm{PhSCl}\right)$. Alternatively, general procedure A was employed to afford $0.260 \mathrm{~g}(90 \%)$ of 12. $R_{\mathrm{f}} 0.18$ (heptane/EtOAc $\left.7: 3\right) .[\alpha]^{25} \mathrm{D}-22\left(c 1.0, \mathrm{CHCl}_{3}\right) .{ }^{1} \mathrm{H} \mathrm{NMR}$ $\left(400 \mathrm{MHz}, \mathrm{CDCl}_{3}\right) \delta 8.02-7.92(\mathrm{~m}, 10 \mathrm{H}), 7.56-7.50(\mathrm{~m}, 5 \mathrm{H}), 7.45-7.27(\mathrm{~m}, 15 \mathrm{H})$, 5.69-5.62 $\left(\mathrm{m}, 2 \mathrm{H}, \mathrm{H} 3^{\mathrm{A}}, \mathrm{H}^{\mathrm{B}}\right), 5.34\left(\mathrm{t}, J=8.0 \mathrm{~Hz}, 1 \mathrm{H}, \mathrm{H} 2^{\mathrm{A}}\right), 5.24\left(\mathrm{dd}, J_{2 \mathrm{~B}, 3 \mathrm{~B}}=6.5 \mathrm{~Hz}, J_{1 \mathrm{~B}, 2 \mathrm{~B}}=5.0 \mathrm{~Hz}, 1 \mathrm{H}\right.$, 
$\left.\mathrm{H} 2^{\mathrm{B}}\right), 5.06-5.01\left(\mathrm{~m}, 2 \mathrm{H}, \mathrm{H} 1^{\mathrm{A}}, \mathrm{H} 4^{\mathrm{B}}\right), 4.95\left(\mathrm{~d}, J_{1 \mathrm{~B}, 2 \mathrm{~B}}=4.8 \mathrm{~Hz}, 1 \mathrm{H}, \mathrm{H} 1^{\mathrm{B}}\right), 4.27\left(\mathrm{dd}, J_{5 \text { Aeq }, 5 \text { Aax }}=\right.$ $\left.12.2 \mathrm{~Hz}, J_{5 \mathrm{Aeq}, 4 \mathrm{~A}}=4.7 \mathrm{~Hz}, 1 \mathrm{H}, \mathrm{H}^{\mathrm{A}}{ }_{\text {eq }}\right), 4.09\left(\mathrm{td}, J=8.3 \mathrm{~Hz}, J_{5 \mathrm{Aeq}, 4 \mathrm{~A}}=4.9 \mathrm{~Hz}, 2 \mathrm{H}, \mathrm{H} 4^{\mathrm{A}}\right), 4.03$ $\left(\mathrm{dd}, J_{5 \mathrm{Beq}, 5 \mathrm{Bax}}=12.4 \mathrm{~Hz}, J_{5 \mathrm{Beq}, 4 \mathrm{~B}}=3.9 \mathrm{~Hz}, 1 \mathrm{H}, \mathrm{H}^{\mathrm{B}}{ }_{\text {eq }}\right), 3.55\left(\mathrm{dd}, J_{5 \mathrm{Aax}, 5 \mathrm{Aeq}}=12.1 \mathrm{~Hz}, J_{5 \mathrm{Aax}, 4 \mathrm{~A}}=\right.$ $\left.8.6 \mathrm{~Hz}, 1 \mathrm{H}, \mathrm{H} 5^{\mathrm{A}}{ }_{\mathrm{ax}}\right), 3.43\left(\mathrm{dd}, J_{5 \mathrm{Bax}, 5 \mathrm{Beq}}=12.4 \mathrm{~Hz}, J_{5 \mathrm{Bax}, 4 \mathrm{~B}}=6.3 \mathrm{~Hz}, 1 \mathrm{H}, \mathrm{H} 5^{\mathrm{B}}{ }_{\mathrm{ax}}\right) .{ }^{13} \mathrm{C}\left\{{ }^{1} \mathrm{H}\right\} \mathrm{NMR}$ $\left(101 \mathrm{MHz}, \mathrm{CDCl}_{3}\right) \delta 165.6,165.5,165.4,165.4,165.2,133.5(\times 2), 133.5,133.4(\times 2), 132.8$, $132.7(\times 2), 130.1(\times 2), 130.0(\times 2), 130.0(\times 2), 129.9(\times 2), 129.9(\times 2), 129.5,129.4,129.3$, $129.2,129.1(\times 2), 129.0,128.6(\times 6), 128.5(\times 4), 128.2,99.8\left(\mathrm{C1}^{\mathrm{B}}\right), 86.8\left(\mathrm{C} 1^{\mathrm{A}}\right), 75.2\left(\mathrm{C} 4^{\mathrm{A}}\right), 73.1$ $\left(\mathrm{C} 3^{\mathrm{A}}\right), 70.6\left(\mathrm{C} 2^{\mathrm{A}}\right), 70.2\left(\mathrm{C}^{\mathrm{B}}\right), 69.7\left(\mathrm{C}^{\mathrm{B}}\right), 68.6\left(\mathrm{C}^{\mathrm{B}}\right), 65.6\left(\mathrm{C}^{\mathrm{A}}\right), 60.9\left(\mathrm{C}^{\mathrm{B}}\right) . \mathrm{HRMS}^{\mathrm{m}} \mathrm{m} / \mathrm{z}$ calcd for $\mathrm{C}_{51} \mathrm{H}_{42} \mathrm{O}_{13} \mathrm{SNa}[\mathrm{M}+\mathrm{Na}]^{+}$917.2238, found 917.2258.

Phenyl 2,3,4-tri-O-benzoyl- $\beta$-D-xylopyranosyl-(1 $\rightarrow 4)$-2,3-di-O-benzoyl- $\beta$-D-xylopyranosyl(1 $\rightarrow 4$ )-2,3-di-O-benzoyl-1-thio- $\beta$-D-xylopyranoside (13). To a Schlenk flask containing $3 \AA ̊$ mol sieves $(1.20 \mathrm{~g})$ was added donor $5(0.200 \mathrm{~g}, 0.36 \mathrm{mmol})$ dissolved in dry $\mathrm{CH}_{2} \mathrm{Cl}_{2}(6.0 \mathrm{~mL})$ and $\operatorname{AgOTf}(0.185 \mathrm{~g}, 0.72 \mathrm{mmol})$ dissolved in $\operatorname{dry} \mathrm{Et}_{2} \mathrm{O}(5.0 \mathrm{~mL})$. The mixture was stirred for $1 \mathrm{~h}$ at rt and then cooled to $-78{ }^{\circ} \mathrm{C}$ at which point $\mathrm{PhSCl}(0.062 \mathrm{~g}, 0.43 \mathrm{mmol})$ or $p \mathrm{NO}_{2} \mathrm{PhSCl}(0.082$ $\mathrm{g}, 0.43 \mathrm{mmol})$ dissolved in dry $\mathrm{CH}_{2} \mathrm{Cl}_{2}(1.0 \mathrm{~mL})$ was added dropwise followed by stirring for 10 min. Acceptor $6(0.146 \mathrm{~g}, 0.33 \mathrm{mmol})$ dissolved in dry $\mathrm{CH}_{2} \mathrm{Cl}_{2}(0.5 \mathrm{~mL})$ was then added slowly. The reaction was stirred at $-78^{\circ} \mathrm{C}$ for $15 \mathrm{~min}$ and then for $15 \mathrm{~min}$ at rt. AgOTf $(0.093 \mathrm{~g}, 0.36$ mmol) dissolved in dry $\mathrm{Et}_{2} \mathrm{O}(2.0 \mathrm{~mL})$ was added and the mixture was cooled to $-78^{\circ} \mathrm{C}$ over 20 min. $\mathrm{PhSCl}(0.058 \mathrm{~g}, 0.40 \mathrm{mmol})$ or $p \mathrm{NO}_{2} \mathrm{PhSCl}(0.075 \mathrm{~g}, 0.40 \mathrm{mmol})$ dissolved in dry $\mathrm{CH}_{2} \mathrm{Cl}_{2}$ $(1.0 \mathrm{~mL})$ was added dropwise followed by stirring for $10 \mathrm{~min}$. Acceptor $\mathbf{6}(0.132 \mathrm{~g}, 0.29 \mathrm{mmol})$ dissolved in dry $\mathrm{CH}_{2} \mathrm{Cl}_{2}(0.4 \mathrm{~mL})$ was added slowly. The reaction was stirred at $-78{ }^{\circ} \mathrm{C}$ for 15 $\mathrm{min}$, then for $15 \mathrm{~min}$ at $\mathrm{rt}$ after which $\mathrm{Et}_{3} \mathrm{~N}(0.4 \mathrm{~mL}, 5.44 \mathrm{mmol})$ was added. The mixture was 
filtered through a pad of Celite, concd in vacuo, and purified by flash column chromatography (pentane/acetone 7.5:2.5) to give a mixture of $\mathbf{1 2}(0.169 \mathrm{~g}, 59 \%)$ and $\mathbf{1 3}(0.134 \mathrm{~g}, 34 \%)$ (with $\mathrm{PhSCl})$ or only 13 as a white amorphous solid $\left(0.249 \mathrm{~g}, 69 \%\right.$, with $\left.p \mathrm{NO}_{2} \mathrm{PhSCl}\right) .{ }^{1} \mathrm{H}$ NMR (400 $\left.\mathrm{MHz}, \mathrm{CDCl}_{3}\right) \delta 7.99-7.89(\mathrm{~m}, 14 \mathrm{H}), 7.57-7.47(\mathrm{~m}, 7 \mathrm{H}), 7.42-7.25(\mathrm{~m}, 19 \mathrm{H}), 5.62-5.57(\mathrm{~m}, 2 \mathrm{H}$, $\left.\mathrm{H}^{\mathrm{A}}, \mathrm{H}^{\mathrm{C}}\right), 5.52\left(\mathrm{t}, J=8.1 \mathrm{~Hz}, 1 \mathrm{H}, \mathrm{H} 3^{\mathrm{B}}\right), 5.31-5.27\left(\mathrm{~m}, 1 \mathrm{H}, \mathrm{H} 2^{\mathrm{A}}\right), 5.19-5.14\left(\mathrm{~m}, 2 \mathrm{H}, \mathrm{H} 2^{\mathrm{B}}\right.$, $\left.\mathrm{H}^{\mathrm{C}}\right), 5.01\left(\mathrm{td}, J=6.4 \mathrm{~Hz}, J=4.1 \mathrm{~Hz}, 1 \mathrm{H}, \mathrm{H}^{\mathrm{C}}\right), 4.97\left(\mathrm{~d}, J=8.1 \mathrm{~Hz}, 1 \mathrm{H}, \mathrm{H} 1^{\mathrm{A}}\right), 4.74-4.71(\mathrm{~m}$, $\left.2 \mathrm{H}, \mathrm{H} 1^{\mathrm{B}}, \mathrm{H} 1^{\mathrm{C}}\right), 4.14\left(\mathrm{dd}, J_{5 \mathrm{Aeq}, 5 \mathrm{Aax}}=12.0 \mathrm{~Hz}, J_{5 \mathrm{Aeq}, 4 \mathrm{~A}}=4.7 \mathrm{~Hz}, 1 \mathrm{H}, \mathrm{H} 5^{\mathrm{A}}\right.$ eq $), 4.00-3.94(\mathrm{~m}, 2 \mathrm{H}$, $\left.\mathrm{H}^{\mathrm{A}}, \mathrm{H}^{\mathrm{C}} \mathrm{C}_{\text {eq }}\right), 3.82\left(\mathrm{td}, J=8.4,5.0 \mathrm{~Hz}, 1 \mathrm{H}, \mathrm{H} 4^{\mathrm{B}}\right), 3.55\left(\mathrm{dd}, J_{5 \mathrm{Beq}, 5 \mathrm{Bax}}=12.3 \mathrm{~Hz}, J_{5 \mathrm{Beq}, 4 \mathrm{~B}}=4.8 \mathrm{~Hz}\right.$, $\left.1 \mathrm{H}, \mathrm{H}^{\mathrm{B}}{ }_{\mathrm{eq}}\right), 3.46\left(\mathrm{dd}, J_{5 \mathrm{Aax}, 5 \mathrm{Aeq}}=12.1 \mathrm{~Hz}, J_{5 \mathrm{Aax}, 4 \mathrm{~A}}=8.7 \mathrm{~Hz}, 1 \mathrm{H}, \mathrm{H}^{\mathrm{A}}{ }_{\mathrm{ax}}\right), 3.35\left(\mathrm{dd}, J_{5 \mathrm{Cax}, 5 \mathrm{Ceq}}=\right.$ $\left.12.3 \mathrm{~Hz}, J_{5 \mathrm{Cax}, 4 \mathrm{C}}=6.3 \mathrm{~Hz}, 1 \mathrm{H}, \mathrm{H}^{\mathrm{C}} \mathrm{Cx}\right), 3.16\left(\mathrm{dd}, J_{5 \mathrm{Bax}, 5 \mathrm{Beq}}=12.3 \mathrm{~Hz}, J_{5 \mathrm{Bax}, 4 \mathrm{~B}}=8.6 \mathrm{~Hz}, 1 \mathrm{H}\right.$, $\left.\mathrm{H} 5^{\mathrm{B}}{ }_{\mathrm{ax}}\right) \cdot{ }^{13} \mathrm{C}\left\{{ }^{1} \mathrm{H}\right\} \mathrm{NMR}\left(101 \mathrm{MHz}, \mathrm{CDCl}_{3}\right) \delta 165.5,165.5,165.5,165.4,165.3,165.2,165.0$, $133.5(\times 2), 133.5,133.5,133.4(\times 2), 133.3,132.8,132.6(\times 2), 130.1(\times 2), 130.0(\times 2), 130.0$ $(\times 2), 129.9(\times 2), 129.9(\times 2), 129.8(\times 2), 129.8(\times 2), 129.6,129.5,129.5,129.3(\times 2), 129.2$, $129.1(\times 2), 129.0,128.6(\times 2), 128.6(\times 2), 128.6(\times 4), 128.5(\times 2), 128.5(\times 2), 128.4(\times 2), 128.2$, $101.1\left(\mathrm{C}^{\mathrm{B}}\right), 99.6\left(\mathrm{C}^{\mathrm{C}}\right), 86.8\left(\mathrm{C1}^{\mathrm{A}}\right), 75.8\left(\mathrm{C} 4^{\mathrm{A}}\right), 74.9\left(\mathrm{C} 4^{\mathrm{B}}\right), 73.1\left(\mathrm{C} 3^{\mathrm{A}}\right), 72.1\left(\mathrm{C} 3^{\mathrm{B}}\right), 71.6\left(\mathrm{C} 2^{\mathrm{B}}\right)$, $70.5\left(\mathrm{C}^{\mathrm{A}}\right), 70.2\left(\mathrm{C}^{\mathrm{C}}\right), 69.7\left(\mathrm{C}^{\mathrm{C}}\right), 68.6\left(\mathrm{C} 4^{\mathrm{C}}\right), 65.9\left(\mathrm{C}^{\mathrm{A}}\right), 62.3\left(\mathrm{C} 5^{\mathrm{B}}\right), 60.9\left(\mathrm{C} 5^{\mathrm{C}}\right)$. HRMS: $m / z$ calcd for $\mathrm{C}_{70} \mathrm{H}_{58} \mathrm{O}_{19} \mathrm{SNa}[\mathrm{M}+\mathrm{Na}]^{+} 1257.3185$, found 1257.3192 .

Phenyl 2,3,4-tri-O-benzoyl- $\beta$-D-xylopyranosyl-(1 $\rightarrow 4)$-2-O-benzoyl-3-O-levulinoyl-1-thio- $\beta$-Dxylopyranoside (14). Disaccharide 14 was obtained in $86 \%$ yield as a light yellow amorphous solid following general procedure A. $R_{\mathrm{f}} 0.42$ (hexane/EtOAc 1:1). $[\alpha]^{25} \mathrm{D}-60.4\left(c 1.0, \mathrm{CHCl}_{3}\right)$. ${ }^{1} \mathrm{H}$ NMR (400 MHz, $\left.\mathrm{CDCl}_{3}\right) \delta 8.05-8.00(\mathrm{~m}, 4 \mathrm{H}), 7.97-7.92(\mathrm{~m}, 4 \mathrm{H}), 7.62-7.45(\mathrm{~m}, 4 \mathrm{H})$, 7.42-7.32 (m, 10H), 7.28-7.25 (m, 3H), $5.70\left(\mathrm{t}, J=6.5 \mathrm{~Hz}, 1 \mathrm{H}, \mathrm{H}^{\mathrm{B}}\right), 5.40(\mathrm{t}, J=8.3 \mathrm{~Hz}, 1 \mathrm{H}$, 


$$
\begin{aligned}
& \left.\mathrm{H}^{\mathrm{A}}\right), 5.28-5.23\left(\mathrm{~m}, 2 \mathrm{H}, \mathrm{H} 2^{\mathrm{B}}, \mathrm{H} 4^{\mathrm{B}}\right), 5.16\left(\mathrm{t}, J=8.4 \mathrm{~Hz}, 1 \mathrm{H}, \mathrm{H} 2^{\mathrm{A}}\right), 4.94\left(\mathrm{~d}, J_{1 \mathrm{~B}, 2 \mathrm{~B}}=4.7 \mathrm{~Hz}, 1 \mathrm{H},\right. \\
& \left.\mathrm{H}^{\mathrm{B}}\right), 4.89\left(\mathrm{~d}, J_{1 \mathrm{~A}, 2 \mathrm{~A}}=8.6 \mathrm{~Hz}, 1 \mathrm{H}, \mathrm{H}^{\mathrm{A}}\right), 4.48\left(\mathrm{dd}, J_{5 \mathrm{Beq}, 5 \mathrm{Bax}}=12.4, \mathrm{~Hz}, J_{5 \mathrm{Beq}, 4 \mathrm{~B}}=3.8 \mathrm{~Hz}, 1 \mathrm{H},\right. \\
& \left.\mathrm{H}^{\mathrm{B}}{ }_{\text {eq }}\right), 4.19\left(\mathrm{dd}, J_{5 \mathrm{Aeq}, 5 \mathrm{Aax}}=12.0 \mathrm{~Hz}, J_{5 \mathrm{Aeq}, 4 \mathrm{~A}}=4.9 \mathrm{~Hz}, 1 \mathrm{H}, \mathrm{H}^{\mathrm{A}} \text { eq }\right), 3.99\left(\mathrm{td}, J=8.7, \mathrm{~Hz}, J_{5 \text { Aeq, } 4 \mathrm{~A}}\right. \\
& \left.=5.0 \mathrm{~Hz}, 1 \mathrm{H}, \mathrm{H}^{\mathrm{A}}\right), 3.75\left(\mathrm{dd}, J_{5 \mathrm{Bax}, 5 \mathrm{Beq}}=12.4 \mathrm{~Hz}, J_{5 \mathrm{Bax}, 4 \mathrm{~B}}=6.1 \mathrm{~Hz}, 1 \mathrm{H}, \mathrm{H}^{\mathrm{B}}{ }_{\mathrm{ax}}\right), 3.44(\mathrm{dd}, \\
& \left.J_{5 \text { Aax }, 5 \text { Aeq }}=12.0 \mathrm{~Hz}, J_{5 \text { Aax }, 4 \mathrm{~A}}=9.1 \mathrm{~Hz}, 1 \mathrm{H}, \mathrm{H}^{\mathrm{A}}{ }_{\mathrm{ax}}\right), 2.65-2.41(\mathrm{~m}, 4 \mathrm{H}), 1.97(\mathrm{~s}, 3 \mathrm{H}) .{ }^{13} \mathrm{C}\left\{{ }^{1} \mathrm{H}\right\} \\
& \text { NMR (101 MHz, } \left.\mathrm{CDCl}_{3}\right) \delta 205.9,171.8,165.6,165.4,165.4,165.2,133.6,133.5(\times 2), 133.5 \text {, } \\
& 132.8(\times 2), 132.5,130.1(\times 2), 130.1(\times 2), 130.1(\times 2), 130.0(\times 2), 129.5,129.4,129.2,129.1, \\
& 129.1(\times 2), 128.6(\times 2), 128.6(\times 2), 128.6(\times 3), 128.2,99.4\left(\mathrm{C1}^{\mathrm{B}}\right), 86.7\left(\mathrm{C} 1^{\mathrm{A}}\right), 74.6\left(\mathrm{C} 4^{\mathrm{A}}\right), 73.2 \\
& \left(\mathrm{C} 3^{\mathrm{A}}\right), 70.7\left(\mathrm{C} 2^{\mathrm{A}}\right), 70.3\left(\mathrm{C}^{\mathrm{B}}\right), 69.6\left(\mathrm{C}^{\mathrm{B}}\right), 68.7\left(\mathrm{C} 4^{\mathrm{B}}\right), 66.1\left(\mathrm{C} 5^{\mathrm{A}}\right), 61.1\left(\mathrm{C}^{\mathrm{B}}\right), \text { 37.9, 29.6, 28.1. } \\
& \text { HRMS: } m / z \text { calcd for } \mathrm{C}_{49} \mathrm{H}_{44} \mathrm{O}_{14} \mathrm{SNa}[\mathrm{M}+\mathrm{Na}]^{+} \text {911.2344, found 911.2340. }
\end{aligned}
$$

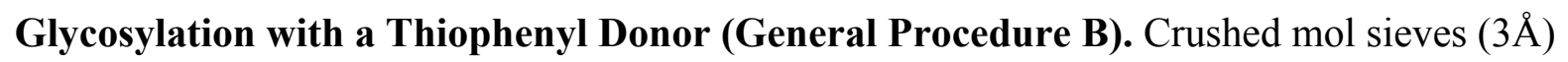
were added to a 2-neck Schlenk flask, where the middle neck was fitted with a glass stopper and the other with a septum. The flask was placed under vacuum, heated with a heatgun and then subjected to an atm of $\mathrm{N}_{2}$ and cooled to rt. The donor (1.0 equiv) dissolved in dry $\mathrm{CH}_{2} \mathrm{Cl}_{2}(c=$ $0.12 \mathrm{M})$ was added to the flask together with $\operatorname{AgOTf}(2.0$ equiv. $)$ dissolved in dry toluene $(c=$ $0.36 \mathrm{M})$. Stirring of the reaction was initiated and the glass stopper was exchanged for a thermometer, and the solution was cooled to $-65^{\circ} \mathrm{C} . p \mathrm{NO}_{2} \mathrm{PhSCl}$ (1.0 equiv) was dissolved in dry $\mathrm{CH}_{2} \mathrm{Cl}_{2}(c=0.72 \mathrm{M})$ and slowly added to avoid raising the temperature above $-60{ }^{\circ} \mathrm{C}$. The mixture was left to stir for approximately $10 \mathrm{~min}$ or until complete activation. The acceptor $(0.9$ equiv.) was dissolved in dry $\mathrm{CH}_{2} \mathrm{Cl}_{2}(c=0.65 \mathrm{M})$ and added quickly. The temperature was kept between $-55^{\circ} \mathrm{C}$ and $-50{ }^{\circ} \mathrm{C}$ until TLC analysis showed completion of the reaction. Afterwards the mixture was allowed to warm to $-15{ }^{\circ} \mathrm{C}$ over $10 \mathrm{~min}$ at which point $\mathrm{Et}_{3} \mathrm{~N}$ (3.0 equiv) was 
added. The solution was filtered through a pad of Celite and concd in vacuo. The residue was then purified by flash column chromatography to afford the product.

Phenyl 2,3,4-tri-O-benzoyl- $\beta$-D-xylopyranosyl- $(1 \rightarrow 4)$-2-O-benzoyl-3-O-levulinoyl- $\beta$ - $D$ xylopyranosyl-(1 $\rightarrow 4)$-2,3-di-O-benzoyl-1-thio- $\beta$-D-xylopyranoside (15). Crushed mol sieves

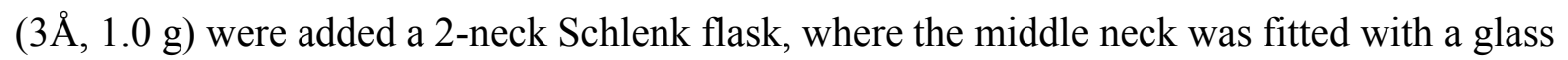
stopper and the other with a septum. The flask was placed under vacuum, heated with a heatgun and then subjected to an atm of $\mathrm{N}_{2}$ and cooled to rt. Donor 5 ( $\left.0.200 \mathrm{~g}, 0.36 \mathrm{mmol}\right)$ dissolved in dry $\mathrm{CH}_{2} \mathrm{Cl}_{2}(3.0 \mathrm{~mL})$ and $\mathrm{AgOTf}(0.185 \mathrm{~g}, 0.72 \mathrm{mmol})$ dissolved in dry toluene $(2.0 \mathrm{~mL})$ were added. Stirring of the reaction was initiated and the glass stopper was exchanged for a thermometer, and the solution was cooled to $-65^{\circ} \mathrm{C} . p \mathrm{NO}_{2} \mathrm{PhSCl}(0.068 \mathrm{~g}, 0.36 \mathrm{mmol})$ dissolved in dry $\mathrm{CH}_{2} \mathrm{Cl}_{2}(0.5 \mathrm{~mL})$ was added dropwise followed by stirring for an additional 10 min. Acceptor $11(0.144 \mathrm{~g}, 0.33 \mathrm{mmol})$ dissolved in dry $\mathrm{CH}_{2} \mathrm{Cl}_{2}(0.5 \mathrm{~mL})$ was quickly added. The reaction was stirred at a temperature between $-55^{\circ} \mathrm{C}$ and $-50{ }^{\circ} \mathrm{C}$ for $15 \mathrm{~min}$ and then for 15 min at rt. The mixture was cooled to $-65^{\circ} \mathrm{C}$. AgOTf $(0.093 \mathrm{~g}, 0.36 \mathrm{mmol})$ dissolved in dry toluene $(1.0 \mathrm{~mL})$ was added followed by stirring for an additional $20 \mathrm{~min} . p \mathrm{NO}_{2} \mathrm{PhSCl}(0.062 \mathrm{~g}$, $0.33 \mathrm{mmol})$ dissolved in dry $\mathrm{CH}_{2} \mathrm{Cl}_{2}(0.4 \mathrm{~mL})$ was added dropwise followed by stirring for 10 min. Acceptor $6(0.132 \mathrm{~g}, 0.29 \mathrm{mmol})$ dissolved in dry $\mathrm{CH}_{2} \mathrm{Cl}_{2}(0.4 \mathrm{~mL})$ was added quickly. The reaction was stirred at a temperature between $-55^{\circ} \mathrm{C}$ and $-50{ }^{\circ} \mathrm{C}$ for $15 \mathrm{~min}$ and then allowed to warm to $-15^{\circ} \mathrm{C}$ over $15 \mathrm{~min}$, at which point $\mathrm{Et}_{3} \mathrm{~N}(0.8 \mathrm{~mL}, 0.54 \mathrm{mmol})$ was added. The mixture was filtered through a pad of Celite, concd in vacuo, and purified by flash column chromatography (heptane/acetone 3:2). The product 15 was obtained as a white amorphous solid $(0.124 \mathrm{~g}, 28 \%)$. Alternatively, general procedure B was employed with crushed mol sieves (1.0 
g), donor 14 (0.490 g, $0.551 \mathrm{mmol}), \operatorname{AgOTf}(0.283 \mathrm{~g}, 1.10 \mathrm{mmol}), p \mathrm{NO}_{2} \mathrm{PhSCl}(0.105 \mathrm{~g}, 0.551$ $\mathrm{mmol})$ and acceptor $6(0.223 \mathrm{~g}, 0.496 \mathrm{mmol})$. Reaction time $15 \mathrm{~min}$ then $\mathrm{Et}_{3} \mathrm{~N}(0.232 \mathrm{~mL}, 1.65$ mmol). Purification by flash column chromatography gave product $15(0.420 \mathrm{~g}, 69 \%) . R_{\mathrm{f}} 0.22$ (heptane/acetone 3:2). $[\alpha]^{25} \mathrm{D}-24.0\left(c\right.$ 1.0, $\left.\mathrm{CHCl}_{3}\right) .{ }^{1} \mathrm{H}$ NMR $\left(400 \mathrm{MHz}, \mathrm{CDCl}_{3}\right) \delta 8.01-7.90$ $(\mathrm{m}, 12 \mathrm{H}), 7.60-7.25(\mathrm{~m}, 23 \mathrm{H}), 5.65\left(\mathrm{t}, J=6.6 \mathrm{~Hz}, 1 \mathrm{H}, \mathrm{H} 3^{\mathrm{C}}\right), 5.58\left(\mathrm{t}, J=7.9 \mathrm{~Hz}, 1 \mathrm{H}, \mathrm{H} 3^{\mathrm{A}}\right)$, $5.31-5.20\left(\mathrm{~m}, 3 \mathrm{H}, \mathrm{H} 2^{\mathrm{A}}, \mathrm{H}^{\mathrm{B}}, \mathrm{H}^{\mathrm{C}}\right), 5.16\left(\mathrm{dd}, J_{2 \mathrm{C}, 3 \mathrm{C}}=6.4 \mathrm{~Hz}, J_{1 \mathrm{C}, 2 \mathrm{C}}=4.9 \mathrm{~Hz}, 1 \mathrm{H}, \mathrm{H} 2^{\mathrm{C}}\right), 5.02$ $\left(\mathrm{dd}, J_{2 \mathrm{~B}, 3 \mathrm{~B}}=8.2 \mathrm{~Hz}, J_{1 \mathrm{~B}, 2 \mathrm{~B}}=6.6 \mathrm{~Hz}, 1 \mathrm{H}, \mathrm{H} 2^{\mathrm{B}}\right), 4.96\left(\mathrm{~d}, J_{1 \mathrm{~A}, 2 \mathrm{~A}}=8.1 \mathrm{~Hz}, 1 \mathrm{H}, \mathrm{H} 1^{\mathrm{A}}\right), 4.71\left(\mathrm{~d}, J_{1 \mathrm{C}, 2 \mathrm{C}}\right.$ $\left.=4.7 \mathrm{~Hz}, 1 \mathrm{H}, \mathrm{H} 1^{\mathrm{C}}\right), 4.66\left(\mathrm{~d}, J_{1 \mathrm{~B}, 2 \mathrm{~B}}=6.4 \mathrm{~Hz}, 1 \mathrm{H}, \mathrm{H}^{\mathrm{B}}\right), 4.40\left(\mathrm{dd}, J_{5 \mathrm{Ceq}, 5 \mathrm{Cax}}=12.4 \mathrm{~Hz}, J_{5 \mathrm{Ceq}, 4 \mathrm{C}}=\right.$ $\left.3.8 \mathrm{~Hz}, 1 \mathrm{H}, \mathrm{H}^{\mathrm{C}} \mathrm{eq}_{\mathrm{eq}}\right), 4.13\left(\mathrm{dd}, J_{5 \mathrm{Aeq}, 5 \mathrm{Aax}}=12.0 \mathrm{~Hz}, J_{5 \mathrm{Aeq}, 4 \mathrm{~A}}=4.7 \mathrm{~Hz}, 1 \mathrm{H}, \mathrm{H} 5^{\mathrm{A}}{ }_{\text {eq }}\right), 3.95(\mathrm{td}, J=8.2$ $\left.\mathrm{Hz}, J_{5 \mathrm{Aeq}, 4 \mathrm{~A}}=4.9 \mathrm{~Hz}, 1 \mathrm{H}, \mathrm{H}^{\mathrm{A}}{ }^{\mathrm{A}}\right), 3.74-3.65\left(\mathrm{~m}, 2 \mathrm{H}, \mathrm{H}^{\mathrm{B}}{ }^{\mathrm{B}}, \mathrm{H}^{\mathrm{C}^{\mathrm{C}}}{ }_{\mathrm{ax}}\right), 3.51\left(\mathrm{dd}, J_{5 \mathrm{Beq}, 5 \mathrm{Bax}}=12.2 \mathrm{~Hz}\right.$, $\left.J_{5 \text { Beq }, 4 \mathrm{~B}}=4.8 \mathrm{~Hz}, 1 \mathrm{H}, \mathrm{H}^{\mathrm{B}}{ }_{\text {eq }}\right), 3.45\left(\mathrm{dd}, J_{5 \mathrm{Aax}, 5 \mathrm{Aeq}}=12.0 \mathrm{~Hz}, J_{5 \mathrm{Aax}, 4 \mathrm{~A}}=8.6 \mathrm{~Hz}, 1 \mathrm{H}, \mathrm{H} 5^{\mathrm{A}}{ }_{\text {ax }}\right), 3.09$ $\left(\mathrm{dd}, J_{5 \mathrm{Bax}, 5 \mathrm{Beq}}=12.2 \mathrm{~Hz}, J_{5 \mathrm{Bax}, 4 \mathrm{~B}}=8.7 \mathrm{~Hz}, 1 \mathrm{H}, \mathrm{H}^{\mathrm{B}}{ }_{\mathrm{ax}}\right), 2.64-2.37\left(\mathrm{~m}, 4 \mathrm{H}, 1.97(\mathrm{~s}, 3 \mathrm{H}) .{ }^{13} \mathrm{C}\left\{{ }^{1} \mathrm{H}\right\}\right.$ NMR (101 MHz, $\left.\mathrm{CDCl}_{3}\right) \delta 205.9,171.8,165.6,165.4,165.3,165.3,165.2,165.0,133.6,133.5$ $(\times 2), 133.5,133.4,133.3,132.8,132.6(\times 2), 130.1(\times 3), 130.0(\times 2), 129.9(\times 2), 129.8(\times 2)$, $129.6,129.4,129.4,129.4,129.3,129.2,129.1(\times 2), 128.6(\times 2), 128.6(\times 3), 128.5(\times 3), 128.5$ $(\times 2), 128.4(\times 2), 128.2,101.0\left(\mathrm{C}^{\mathrm{B}}\right), 99.1\left(\mathrm{C} 1^{\mathrm{C}}\right), 86.7\left(\mathrm{C}^{\mathrm{A}}\right), 75.6\left(\mathrm{C} 4^{\mathrm{A}}\right), 74.2\left(\mathrm{C} 4^{\mathrm{B}}\right), 73.1$ $\left(\mathrm{C} 3^{\mathrm{A}}\right), 71.9\left(\mathrm{C}^{\mathrm{B}}\right), 71.6\left(\mathrm{C} 2^{\mathrm{B}}\right), 70.4\left(\mathrm{C} 2^{\mathrm{A}}\right), 70.1\left(\mathrm{C} 2^{\mathrm{C}}\right), 69.6\left(\mathrm{C} 3^{\mathrm{C}}\right), 68.7\left(\mathrm{C} 4^{\mathrm{C}}\right), 65.9\left(\mathrm{C}^{\mathrm{A}}\right), 62.2$ $\left(\mathrm{C}^{\mathrm{B}}\right), 61.0\left(\mathrm{C}^{\mathrm{C}}\right), 37.8,29.6,28.0 . \mathrm{HRMS}: \mathrm{m} / z$ calcd. for $\mathrm{C}_{68} \mathrm{H}_{60} \mathrm{O}_{20} \mathrm{SNa}[\mathrm{M}+\mathrm{Na}]^{+}$1251.3291, found 1251.3308 .

2,3,5-Tri-O-benzoyl-L-arabinofuranosyl N-phenyl-2,2,2-trifluoroacetimidate (4). AcCl (5.0 mL, $70.3 \mathrm{mmol})$ was slowly added to methanol $(60.0 \mathrm{~mL})$ cooled to $0{ }^{\circ} \mathrm{C}$. The methanolic $\mathrm{HCl}$ solution was then added to a vigorously stirred suspension of L-arabinose (10.0 g, $66.8 \mathrm{mmol})$ in 
methanol $(200 \mathrm{~mL})$. The reaction was left to stir at $\mathrm{rt}$ for $4.5 \mathrm{~h}$, then the mixture was concd under co-evaporation with $\mathrm{CH}_{2} \mathrm{Cl}_{2}$. The resulting residue was cooled to $0{ }^{\circ} \mathrm{C}$ and dissolved in pyridine $(60.0 \mathrm{~mL}) . \mathrm{BzCl}(62.0 \mathrm{~mL}, 534 \mathrm{mmol})$ was slowly added over $25 \mathrm{~min}$ and the reaction was left to stir at rt overnight. $\mathrm{H}_{2} \mathrm{O}(5.0 \mathrm{~mL})$ was added to quench the reaction which was stirred for an additional $5 \mathrm{~min}$. The mixture was then diluted with $\mathrm{CH}_{2} \mathrm{Cl}_{2}(500 \mathrm{~mL})$ and washed with $\mathrm{H}_{2} \mathrm{O}, 1 \mathrm{M} \mathrm{HCl}$, and $\mathrm{NaHCO}_{3}$, dried over $\mathrm{MgSO}_{4}$, filtered, and concd in vacuo under coevaporation with toluene. The residue was crystallized from absolute EtOH with the addition of pentane to promote the crystallization. The intermediate methyl 2,3,5-tri- $O$-benzoyl- $\alpha$-Larabinofuranoside was obtained as white crystals (14.13 g, $44 \%$ over 2 steps). $R_{\mathrm{f}} 0.44$ (hexane/EtOAc 2:1). Mp 95.3-97.7 ${ }^{\circ} \mathrm{C}(\mathrm{EtOH})\left(\right.$ lit. $\left.{ }^{35} 106{ }^{\circ} \mathrm{C}\right) .{ }^{1} \mathrm{H}$ NMR $\left(400 \mathrm{MHz}, \mathrm{CDCl}_{3}\right) \delta$ 8.09-8.00 (m, 6H), 7.62-7.56 (m, 2H), 7.53-7.49 (m, 1H), $7.46(\mathrm{t}, J=7.7 \mathrm{~Hz}, 2 \mathrm{H}), 7.40(\mathrm{t}, J=$ $7.8 \mathrm{~Hz}, 2 \mathrm{H}), 7.30$ (t, $J=7.8 \mathrm{~Hz}, 2 \mathrm{H}), 5.59$ (d, $J=5.2 \mathrm{~Hz}, 1 \mathrm{H}, \mathrm{H} 3), 5.52$ (d, $J=1.3 \mathrm{~Hz}, 1 \mathrm{H}, \mathrm{H} 2)$, $5.18(\mathrm{~s}, 1 \mathrm{H}, \mathrm{H} 1), 4.85\left(\mathrm{dd}, J_{5,5},=11.9 \mathrm{~Hz}, J_{4,5}=3.4 \mathrm{~Hz}, 1 \mathrm{H}, \mathrm{H} 5\right), 4.70\left(\mathrm{dd}, J_{5},{ }_{5}=11.9 \mathrm{~Hz}, J_{4,5},=\right.$ $4.8 \mathrm{~Hz}, 1 \mathrm{H}, \mathrm{H} 5$ '), 4.59-4.56 (m, 1H, H4), 3.50 (s, 3H, OMe). ${ }^{13} \mathrm{C}\left\{{ }^{1} \mathrm{H}\right\} \mathrm{NMR}\left(101 \mathrm{MHz}, \mathrm{CDCl}_{3}\right)$ $\delta 166.4,166.0,165.6,133.7,133.6,133.2,130.1(\times 2), 130.0(\times 2), 129.9(\times 2), 129.2,129.2$, $128.7(\times 2), 128.6(\times 2), 128.5(\times 2), 107.0(\mathrm{C} 1), 82.4(\mathrm{C} 2), 81.0(\mathrm{C} 4), 78.1(\mathrm{C} 3), 63.9(\mathrm{C} 5), 55.2$ (OMe). NMR data are in accordance with literature values. ${ }^{35}$ The above glycoside $(1.92 \mathrm{~g}, 4.03$ mmol) was dissolved in $80 \% \mathrm{AcOH}(13.0 \mathrm{~mL})$ and $33 \% \mathrm{HBr} \cdot \mathrm{AcOH}(11.0 \mathrm{~mL}, 60.7 \mathrm{mmol})$ was added under an atm of $\mathrm{N}_{2}$. The solution was let stir at $\mathrm{rt}$ for $3.5 \mathrm{~h}$. EtOAc was added and the mixture washed with brine and $\mathrm{H}_{2} \mathrm{O}$, dried over $\mathrm{MgSO}_{4}$, filtered, and concd in vacuo. The residue was purified by flash column chromatography (hexane/EtOAc 7:1) to afford 2,3,5-tri-Obenzoyl-L-arabinofuranose ( $\alpha: \beta$ ratio $1: 0.35)$ as a white foam $(1.445 \mathrm{~g}, 77 \%) . R_{\mathrm{f}} 0.41$ (hexane/EtOAc 2:1). ${ }^{1} \mathrm{H}$ NMR (400 MHz, $\left.\mathrm{CDCl}_{3}\right) \delta 8.13-8.03(\mathrm{~m}, 8 \mathrm{H}), 7.66-7.32(\mathrm{~m}, 12 \mathrm{H})$, 
$5.91\left(\mathrm{t}, J=5.4 \mathrm{~Hz}, 0.35 \mathrm{H}, \mathrm{H} 3_{\beta}\right), 5.86\left(\mathrm{~d}, J=4.4 \mathrm{~Hz}, 0.34 \mathrm{H}, \mathrm{H} 1_{\beta}\right), 5.71\left(\mathrm{~s}, 1 \mathrm{H}, \mathrm{H} 1_{\alpha}\right), 5.61-5.57$ (m, 2.35H, H2 $\left.{ }_{\alpha}, \mathrm{H} 3_{\alpha}, \mathrm{H} 2_{\beta}\right), 4.89-4.75\left(\mathrm{~m}, 2.70 \mathrm{H}, \mathrm{H} 4_{\alpha}, \mathrm{H} 5_{\alpha}, \mathrm{H} 5_{\beta}, \mathrm{H} 5^{\prime}{ }_{\beta}\right), 4.69$ (dd, $J=11.5,5.2$ $\left.\mathrm{Hz}, 1 \mathrm{H}, \mathrm{H} 5{ }_{\alpha}{ }_{\alpha}\right), 4.51-4.47\left(\mathrm{~m}, 0.34 \mathrm{H}, \mathrm{H} 4_{\beta}\right) .{ }^{13} \mathrm{C}\left\{{ }^{1} \mathrm{H}\right\} \mathrm{NMR}\left(101 \mathrm{MHz}, \mathrm{CDCl}_{3}\right) \delta 166.6,166.4$, $166.1,166.0,165.9,165.7,134.0,133.8,133.7,133.7,133.3,133.3,132.2,130.3,130.1,130.1$, 130.0, 129.9, $129.9129 .8,129.1,129.1,128.7,128.7,128.6,128.5,128.5,128.4,101.2\left(\mathrm{C} 1_{\alpha}\right)$ $95.7\left(\mathrm{Cl}_{\beta}\right), 82.6\left(\mathrm{C} 2_{\alpha}\right), 81.6\left(\mathrm{C} 4_{\alpha}\right), 79.3\left(\mathrm{C} 4_{\beta}\right), 78.1\left(\mathrm{C} 3_{\alpha}\right), 77.8\left(\mathrm{C} 2_{\beta}\right), 76.7\left(\mathrm{C} 3_{\beta}\right), 65.9\left(\mathrm{C} 5_{\beta}\right)$, $64.1\left(\mathrm{C} 5_{\alpha}\right)$. NMR data are in accordance with literature values. ${ }^{19 \mathrm{a}}$ The above hemiacetal $(0.500$ $\mathrm{g}, 1.08 \mathrm{mmol})$ was dissolved in acetone $(4.0 \mathrm{~mL})$ followed by addition of $\mathrm{Cs}_{2} \mathrm{CO}_{3}(0.704 \mathrm{~g}, 2.16$ mmol) and 2,2,2-trifluoro- $N$-phenylacetimidoyl chloride $(0.35 \mathrm{~mL}, 2.21 \mathrm{mmol})$. The mixture was left to stir at $\mathrm{rt}$ for $2 \mathrm{~h}$ and then filtered through a pad of Celite, concd in vacuo, and purified by flash column chromatography (hexane/EtOAc 10:1). The product 4 was obtained a yellow foam. It was possible to separate some of the $\alpha$ isomer $(0.281 \mathrm{~g}, 41 \%)$ from the mixture $(\alpha / \beta$ ratio 1:2) (0.292 $\mathrm{g}, 43 \%$ ) (overall $\alpha / \beta$ ratio $2: 1) . R_{\mathrm{f}}(\alpha) 0.57$ and $R_{\mathrm{f}}(\beta) 0.52$ (hexane/EtOAc $3: 1$ ). ${ }^{1} \mathrm{H}$ NMR $\left(400 \mathrm{MHz}, \mathrm{CDCl}_{3}\right) \delta 8.10-8.08(\mathrm{~m}, 5 \mathrm{H}), 8.06-8.04(\mathrm{~m}, 3 \mathrm{H}), 8.01(\mathrm{~d}, J=7.5 \mathrm{~Hz}, 1 \mathrm{H})$, 7.66-7.38 (m, 11.5H), 7.32-7.26 (m, 3H), $7.17(\mathrm{t}, J=7.8 \mathrm{~Hz}, 2 \mathrm{H}), 7.09(\mathrm{t}, J=7.5 \mathrm{~Hz}, 0.5 \mathrm{H})$, $7.03(\mathrm{t}, J=7.5 \mathrm{~Hz}, 1 \mathrm{H}), 6.88(\mathrm{~d}, J=7.8 \mathrm{~Hz}, 1 \mathrm{H}), 6.83\left(\mathrm{bs}, 1 \mathrm{H}, \mathrm{H} 1_{\beta}\right), 6.58(\mathrm{~d}, J=7.7 \mathrm{~Hz}, 2 \mathrm{H})$, 6.02-5.99 (m, $\left.1 \mathrm{H}, \mathrm{H} 3_{\beta}\right), 5.93-5.91\left(\mathrm{~m}, 1 \mathrm{H}, \mathrm{H} 2_{\beta}\right), 5.80\left(\mathrm{~s}, 0.5 \mathrm{H}, \mathrm{H} 2_{\alpha}\right), 5.67-5.66(\mathrm{~m}, 0.5 \mathrm{H}$, $\left.\mathrm{H} 3_{\alpha}\right), 4.84-4.80\left(\mathrm{~m}, 2 \mathrm{H}, \mathrm{H} 4_{\alpha}, \mathrm{H} 5_{\alpha}, \mathrm{H} 5_{\beta}\right), 4.76-4.71\left(\mathrm{~m}, 1.5 \mathrm{H}, \mathrm{H} 5^{\prime}{ }_{\alpha}, \mathrm{H} 5^{\prime}{ }_{\beta}\right), 4.67-4.63(\mathrm{~m}, 1 \mathrm{H}$, $\left.\mathrm{H} 4_{\beta}\right) .{ }^{13} \mathrm{C}\left\{{ }^{1} \mathrm{H}\right\} \mathrm{NMR}\left(101 \mathrm{MHz}, \mathrm{CDCl}_{3}\right) \delta 166.3,166.2,165.9,165.7,165.6,165.2,143.5$, $143.4,134.0,134.0(\times 2), 133.9(\times 2), 133.3(\times 2), 130.1(\times 4), 130.1(\times 4), 130.0(\times 4), 129.9(\times 4)$, $129.7,129.7,129.0,128.9(\times 2), 128.8(\times 4), 128.7,128.5(\times 4), 124.6,124.3,119.7,119.3,102.4$ $\left(\mathrm{C} 1_{\alpha}\right), 97.0\left(\mathrm{C} 1_{\beta}\right), 84.4\left(\mathrm{C} 4_{\alpha}\right), 80.9\left(\mathrm{C} 2_{\alpha}\right), 80.7\left(\mathrm{C} 4_{\beta}\right), 77.4\left(\mathrm{C} 3_{\alpha}\right), 76.3\left(\mathrm{C} 2_{\beta}\right), 75.7\left(\mathrm{C} 3_{\beta}\right), 65.0$ 
$\left(\mathrm{C} 5_{\beta}\right), 63.7\left(\mathrm{C}_{\alpha}\right)$. HRMS: $m / z$ calcd for $\mathrm{C}_{34} \mathrm{H}_{26} \mathrm{~F}_{3} \mathrm{NO}_{8} \mathrm{Na}[\mathrm{M}+\mathrm{Na}]^{+}$656.1503, found 656.1499. NMR data are in accordance with literature values. ${ }^{19}$

Deprotection of Levulinoyl Groups (General Procedure C). The linear xylan saccharide (1.0 equiv) was dissolved in $\mathrm{AcOH}(c=0.40 \mathrm{M})$ and pyridine $(c=0.20 \mathrm{M})$. A mixture of a $50 \%$ solution of hydrazine hydrate (10.0 equiv) in $\mathrm{AcOH}(c=20 \mathrm{M})$ and pyridine $(c=10 \mathrm{M})$ was added. The reaction was left to stir at rt until TLC showed consumption of the starting material and formation of the product. The reaction was stopped by the addition of acetone (300 equiv) and left to stir at rt for $30 \mathrm{~min}$. EtOAc was added and the mixture was washed with $1 \mathrm{M} \mathrm{HCl}$, $\mathrm{NaHCO}_{3}$, and $\mathrm{H}_{2} \mathrm{O}$, dried over $\mathrm{Na}_{2} \mathrm{SO}_{4}$, filtered, and concd in vacuo. The product was obtained in sufficient purity and did not need further purification.

Glycosylation with $N$-Phenyl-2,2,2-trifluoroacetimidate 4 (General Procedure D). The partially deprotected xylan (1.0 equiv) and donor 4 (4.0 equiv) were co-evaporated twice with toluene and left under high vacuum overnight. The mixture was dissolved in dry $\mathrm{CH}_{2} \mathrm{Cl}_{2}(20$ $\mathrm{mL} / 0.1 \mathrm{~g}$ deprotected xylan) and cooled to $-40{ }^{\circ} \mathrm{C}$. TMSOTf ( 0.1 equiv) was added from a freshly made stock solution of TMSOTf in dry $\mathrm{CH}_{2} \mathrm{Cl}_{2}$. The reaction was kept at $-40{ }^{\circ} \mathrm{C}$ for the time indicated after which $\mathrm{Et}_{3} \mathrm{~N}$ (0.7 equiv) was added, and the mixture was concd in vacuo. The residue was purified by flash column chromatography to afford the desired product.

\section{Deprotection of Anomeric Thiophenyl Group to Prepare 29 - 31 (General Procedure E).}

The arabinoxylan saccharide (1.0 equiv) was dissolved in acetone/ $\mathrm{H}_{2} \mathrm{O} 9: 1(15 \mathrm{~mL} / \mathrm{mmol})$ and NBS (4.0 equiv) was added. The mixture was left to stir at rt until TLC analysis showed 
completion of the reaction. EtOAc was added to the mixture, which was then washed with $\mathrm{NaHCO}_{3}$ and $\mathrm{H}_{2} \mathrm{O}$, dried over $\mathrm{Na}_{2} \mathrm{SO}_{4}$, filtered, and concd in vacuo. The residue was purified by flash column chromatography.

Deprotection of Benzoyl Groups (General Procedure F). The arabinoxylan saccharide (1.0 equiv) was dissolved in $\mathrm{CH}_{2} \mathrm{Cl}_{2} / \mathrm{MeOH}$ 1:1 $(c=0.01 \mathrm{M})$. A $1 \mathrm{M} \mathrm{NaOMe}$ solution was added until the solution had obtained a $\mathrm{pH}$ of 11 . The reaction was stirred at $\mathrm{rt}$ until TLC analysis (EtOAc/MeOH/H $\mathrm{H}_{2} \mathrm{O} / \mathrm{AcOH}$ 6:3:0.8:0.2) showed completion of the reaction (about $1-3 \mathrm{~h}$ ). The mixture was diluted with water and washed with $\mathrm{Et}_{2} \mathrm{O}$ and EtOAc and concd followed by purification with Sep-Pak C18 Plus Short Cartridge (Waters) with a gradient of $\mathrm{H}_{2} \mathrm{O}$ to $\mathrm{H}_{2} \mathrm{O} / \mathrm{MeOH} 1: 1$.

\section{Deprotection of Anomeric Thiophenyl Group to Prepare 1 - 3 (General Procedure G). The} partially deprotected arabinoxylan saccharide (1.0 equiv) was dissolved in $\mathrm{MeCN} / \mathrm{H}_{2} \mathrm{O}$ 5:1 ( $c=$ $0.02 \mathrm{M}$ ) followed by addition of 2,6-lutidine (1.5 equiv) and NBS (3.0 equiv). The mixture was stirred at rt until TLC analysis (EtOAc/MeOH/ $\mathrm{H}_{2} \mathrm{O} / \mathrm{AcOH}$ 6:3:0.8:0.2) showed completion of the reaction. The mixture was concd in vacuo. The crude residue was dissolved in $\mathrm{MeOH}(0.5$ $\mathrm{mL}$ ) followed by addition of ice-cold $\mathrm{Et}_{2} \mathrm{O}$, and precipitation for $30 \mathrm{~min}$ in the freezer. The mixture was then centrifuged and the supernatant removed. The precipitation process was repeated three times in total. The resulting residue was purified by gel filtration (PD miniTrap G-10 column). 
Phenyl 2,3,4-tri-O-benzoyl- $\beta$-D-xylopyranosyl-(1 $\rightarrow 4)$-2-O-benzoyl-3-O-levulinoyl- $\beta$-Dxylopyranosyl-(l $\rightarrow 4$ )-2-O-benzoyl-3-O-levulinoyl-1-thio- $\beta$-D-xylopyranoside (16). General procedure B with crushed mol sieves (1.0 g), donor 14 ( $0.250 \mathrm{~g}, 0.28 \mathrm{mmol})$, AgOTf $(0.145 \mathrm{~g}$, $0.56 \mathrm{mmol}), p \mathrm{NO}_{2} \mathrm{PhSCl}(0.053 \mathrm{~g}, 0.28 \mathrm{mmol})$ and acceptor 11 (0.113 g, $\left.0.25 \mathrm{mmol}\right)$. Reaction time $15 \mathrm{~min}$ then $\mathrm{Et}_{3} \mathrm{~N}(0.12 \mathrm{~mL}, 0.84 \mathrm{mmol})$. Purification by flash column chromatography (heptane/EtOAc 4:3) gave $\mathbf{1 6}$ as a white amorphous solid (0.274 g, 88\%). $R_{\mathrm{f}} 0.37$ (heptane/EtOAc 1:1). $[\alpha]^{25}{ }_{\mathrm{D}}-57.7\left(c\right.$ 1.0, $\left.\mathrm{CHCl}_{3}\right) .{ }^{1} \mathrm{H}$ NMR $\left(400 \mathrm{MHz}, \mathrm{CDCl}_{3}\right) \delta 8.05-7.95(\mathrm{~m}$, 10H), 7.63-7.46 (m, 9H), 7.43-7.35 (m, 8H), 7.29-7.25 (m, 3H), $5.73\left(\mathrm{t}, J=6.6 \mathrm{~Hz}, 1 \mathrm{H}, \mathrm{H}^{\mathrm{C}}\right)$, $5.37-5.26\left(\mathrm{~m}, 4 \mathrm{H}, \mathrm{H}^{\mathrm{A}}, \mathrm{H}^{\mathrm{B}}, \mathrm{H}^{\mathrm{C}}, \mathrm{H} 4^{\mathrm{C}}\right), 5.13\left(\mathrm{t}, J_{1 \mathrm{~A}, 2 \mathrm{~A}}=8.3 \mathrm{~Hz}, 1 \mathrm{H}, \mathrm{H} 2^{\mathrm{A}}\right), 5.05\left(\mathrm{dd}, J_{2 \mathrm{~B}, 3 \mathrm{~B}}=\right.$ $\left.8.3 \mathrm{~Hz}, J_{1 \mathrm{~B}, 2 \mathrm{~B}}=6.5 \mathrm{~Hz}, 1 \mathrm{H}, \mathrm{H} 2^{\mathrm{B}}\right), 4.96\left(\mathrm{~d}, J_{1 \mathrm{C}, 2 \mathrm{C}}=4.7 \mathrm{~Hz}, 1 \mathrm{H}, \mathrm{H} 1^{\mathrm{C}}\right), 4.86\left(\mathrm{~d}, J_{1 \mathrm{~A}, 2 \mathrm{~A}}=8.4 \mathrm{~Hz}\right.$ $\left.1 \mathrm{H}, \mathrm{H} 1^{\mathrm{A}}\right), 4.67\left(\mathrm{~d}, J_{1 \mathrm{~B}, 2 \mathrm{~B}}=6.4 \mathrm{~Hz}, 1 \mathrm{H}, \mathrm{H1}^{\mathrm{B}}\right), 4.48\left(\mathrm{dd}, J_{5 \mathrm{Ceq}, 5 \mathrm{Cax}}=12.4 \mathrm{~Hz}, J_{5 \mathrm{Ceq}, 4 \mathrm{C}}=3.9 \mathrm{~Hz}, 1 \mathrm{H}\right.$, $\left.\mathrm{H}^{\mathrm{C}}{ }_{\mathrm{eq}}\right), 4.11-4.04\left(\mathrm{~m}, 2 \mathrm{H}, \mathrm{H} 5^{\mathrm{A}} \mathrm{eq}, \mathrm{H}^{\mathrm{B}}{ }_{\mathrm{eq}}\right), 3.98-3.97\left(\mathrm{~m}, 1 \mathrm{H}, \mathrm{H} 4^{\mathrm{B}}\right), 3.88-3.82\left(\mathrm{~m}, 1 \mathrm{H}, \mathrm{H} 4^{\mathrm{A}}\right), 3.77$ $\left(\mathrm{dd}, J_{5 \mathrm{Cax}, 5 \mathrm{Ceq}}=12.4 \mathrm{~Hz}, J_{5 \mathrm{Cax}, 4 \mathrm{C}}=6.2 \mathrm{~Hz}, 1 \mathrm{H}, \mathrm{H} 5_{\mathrm{ax}}^{\mathrm{C}}\right), 3.42\left(\mathrm{dd}, J_{5 \mathrm{Bax}, 5 \mathrm{Beq}}=12.1 \mathrm{~Hz}, J_{5 \mathrm{Bax}, 4 \mathrm{~B}}=8.4\right.$ $\left.\mathrm{Hz}, 1 \mathrm{H}, \mathrm{H}^{\mathrm{B}}{ }_{\mathrm{ax}}\right), 3.36\left(\mathrm{dd}, J_{5 \mathrm{Aax}, 5 \mathrm{Aeq}}=11.9 \mathrm{~Hz}, J_{5 \mathrm{Aax}, 4 \mathrm{~A}}=9.0 \mathrm{~Hz}, 1 \mathrm{H}, \mathrm{H}^{\mathrm{A}}{ }_{\mathrm{ax}}\right), 2.66-2.39(\mathrm{~m}, 8 \mathrm{H})$, $2.06(\mathrm{~s}, 3 \mathrm{H}), 2.01(\mathrm{~s}, 3 \mathrm{H}) \cdot{ }^{13} \mathrm{C}\left\{{ }^{1} \mathrm{H}\right\}$ NMR $\left(101 \mathrm{MHz}, \mathrm{CDCl}_{3}\right) \delta 206.0,205.9,171.8$, 171.6, 165.6, 165.3, 165.3, $165.1(\times 2), 133.5,133.5,133.4,133.4,132.6(\times 2), 132.6,130.1(\times 2), 130.0$ $(\times 2), 130.0(\times 2), 129.9(\times 2), 129.9(\times 2), 129.5,129.4,129.4,129.2,129.1,129.0(\times 2), 128.6$ $(\times 2), 128.5(\times 4), 128.5(\times 4), 128.1,100.5\left(\mathrm{C}^{\mathrm{B}}\right), 99.2\left(\mathrm{C1}^{\mathrm{C}}\right), 86.5\left(\mathrm{C} 1^{\mathrm{A}}\right), 74.9\left(\mathrm{C} 4^{\mathrm{A}}\right), 74.3\left(\mathrm{C} 4^{\mathrm{B}}\right)$, $73.0\left(\mathrm{C}^{\mathrm{A}}\right), 71.9\left(\mathrm{C}^{\mathrm{B}}\right), 71.4\left(\mathrm{C} 2^{\mathrm{B}}\right), 70.4,70.2\left(\mathrm{C}^{\mathrm{A}}, \mathrm{C}^{\mathrm{C}}\right), 69.7\left(\mathrm{C} 3^{\mathrm{C}}\right), 68.7\left(\mathrm{C} 4^{\mathrm{C}}\right), 66.1\left(\mathrm{C} 5^{\mathrm{A}}\right)$, $62.4\left(\mathrm{C}^{\mathrm{B}}\right), 61.1\left(\mathrm{C}^{\mathrm{C}}\right), 37.8,37.8,29.8,29.6,28.0,28.0$. HRMS: $m / z$ calcd for $\mathrm{C}_{66} \mathrm{H}_{62} \mathrm{O}_{21} \mathrm{SNa}$ $[\mathrm{M}+\mathrm{Na}]^{+}$1245.3396, found 1245.3379. 
Phenyl 2,3,4-tri-O-benzoyl- $\beta$-D-xylopyranosyl-(1 $\rightarrow 4)$-2-O-benzoyl-3-O-levulinoyl- $\beta$-Dxylopyranosyl-( $(1 \rightarrow 4)$-2-O-benzoyl-3-O-levulinoyl- $\beta$-D-xylopyranosyl-( $(\rightarrow \rightarrow 4)-2-O$-benzoyl-3-Olevulinoyl-1-thio- $\beta$-D-xylopyranoside (17). General procedure B with crushed mol sieves (1.5 g), donor 16 (0.794 g, $0.65 \mathrm{mmol})$, AgOTf (0.332 g, $1.29 \mathrm{mmol}), p \mathrm{NO}_{2} \mathrm{PhSCl}(0.123 \mathrm{~g}, 0.65 \mathrm{mmol})$ and acceptor 11 (0.262 g, $0.59 \mathrm{mmol})$. Reaction time $60 \mathrm{~min}$ then $\mathrm{Et}_{3} \mathrm{~N}(0.27 \mathrm{~mL}, 1.95 \mathrm{mmol})$. Purification by flash column chromatography (heptane/acetone 2:1) gave $\mathbf{1 7}$ as a white amorphous solid (0.670 g, 73\%). $R_{\mathrm{f}} 0.34$ (hexane/acetone 1:1). $[\alpha]^{25} \mathrm{D}-46.6\left(c 1.0, \mathrm{CHCl}_{3}\right) .{ }^{1} \mathrm{H}$ NMR (400 MHz, $\left.\mathrm{CDCl}_{3}\right) \delta 8.01-7.92(\mathrm{~m}, 12 \mathrm{H}), 7.60-7.31(\mathrm{~m}, 20 \mathrm{H}), 7.25-7.21(\mathrm{~m}, 3 \mathrm{H}), 5.70$ $\left(\mathrm{t}, J=6.5 \mathrm{~Hz}, 1 \mathrm{H}, \mathrm{H}^{\mathrm{D}}\right), 5.32\left(\mathrm{t}, J_{2 \mathrm{~B}, 3 \mathrm{~B}}=8.2 \mathrm{~Hz}, 1 \mathrm{H}, \mathrm{H} 3_{\mathrm{B}}\right), 5.28-5.24\left(\mathrm{~m}, 3 \mathrm{H}, \mathrm{H}^{\mathrm{A}}, \mathrm{H} 2^{\mathrm{D}}, \mathrm{H} 4^{\mathrm{D}}\right)$, $5.20\left(\mathrm{t}, J_{2 \mathrm{C}, 3 \mathrm{C}}=8.0 \mathrm{~Hz}, 1 \mathrm{H}, \mathrm{H}^{\mathrm{C}}\right), 5.08\left(\mathrm{t}, J_{1 \mathrm{~A}, 2 \mathrm{~A}}=8.3 \mathrm{~Hz}, 1 \mathrm{H}, \mathrm{H} 2^{\mathrm{A}}\right), 5.04\left(\mathrm{dd}, J_{2 \mathrm{~B}, 3 \mathrm{~B}}=8.4 \mathrm{~Hz}\right.$, $\left.J_{1 \mathrm{~B}, 2 \mathrm{~B}}=6.5 \mathrm{~Hz}, 1 \mathrm{H}, \mathrm{H} 2^{\mathrm{B}}\right), 4.97\left(\mathrm{dd}, J_{2 \mathrm{C}, 3 \mathrm{C}}=8.3 \mathrm{~Hz}, J_{1 \mathrm{C}, 2 \mathrm{C}}=6.4 \mathrm{~Hz}, 1 \mathrm{H}, \mathrm{H} 2^{\mathrm{C}}\right), 4.93\left(\mathrm{~d}, J_{1 \mathrm{D}, 2 \mathrm{D}}=\right.$ $\left.4.7 \mathrm{~Hz}, 1 \mathrm{H}, \mathrm{H1}^{\mathrm{D}}\right), 4.81\left(\mathrm{~d}, J_{1 \mathrm{~A}, 2 \mathrm{~A}}=8.4 \mathrm{~Hz}, 1 \mathrm{H}, \mathrm{H}^{\mathrm{A}}\right), 4.64\left(\mathrm{~d}, J_{1 \mathrm{~B}, 2 \mathrm{~B}}=6.5 \mathrm{~Hz}, 1 \mathrm{H}, \mathrm{H} 1^{\mathrm{B}}\right), 4.57(\mathrm{~d}$, $\left.J_{1 \mathrm{C}, 2 \mathrm{C}}=6.3 \mathrm{~Hz}, 1 \mathrm{H}, \mathrm{H} 1^{\mathrm{C}}\right), 4.46\left(\mathrm{dd}, J_{5 \mathrm{Deq}, 5 \mathrm{Dax}}=12.4 \mathrm{~Hz}, J_{5 \mathrm{Deq}, 4 \mathrm{D}}=3.9 \mathrm{~Hz}, 1 \mathrm{H}, \mathrm{H} 5^{\mathrm{D}}\right.$ eq $), 4.05-4.00$ (m, 2H, H5 $5^{\mathrm{A}}, \mathrm{H} 5^{\mathrm{B}}{ }_{\text {eq }}$ ), 3.95-3.89 (m, 2H, H4 $\left.{ }^{\mathrm{B}}, \mathrm{H} 5^{\mathrm{C}}\right), 3.80-3.72\left(\mathrm{~m}, 3 \mathrm{H}, \mathrm{H} 4^{\mathrm{A}}, \mathrm{H} 4^{\mathrm{C}}, \mathrm{H}^{\mathrm{D}}{ }_{\mathrm{ax}}\right), 3.38$ $\left(\mathrm{dd}, J_{5 \mathrm{Bax}, 5 \mathrm{Beq}}=12.1 \mathrm{~Hz}, J_{5 \mathrm{Bax}, 4 \mathrm{~B}}=8.5 \mathrm{~Hz}, 1 \mathrm{H}, \mathrm{H} 5^{\mathrm{B}}{ }_{\mathrm{ax}}\right), 3.33-3.25\left(\mathrm{~m}, 2 \mathrm{H}, \mathrm{H} 5^{\prime}{ }^{\mathrm{A}}, \mathrm{H}^{\prime}{ }^{\prime} \mathrm{C}\right)$, 2.66-2.32 (m, 12H), 2.03 (s, 3H), $2.01(\mathrm{~s}, 3 \mathrm{H}), 1.97(\mathrm{~s}, 3 \mathrm{H}) .{ }^{13} \mathrm{C}\left\{{ }^{1} \mathrm{H}\right\}$ NMR (101 MHz, $\left.\mathrm{CDCl}_{3}\right)$ $\delta 206.1,206.0,205.9,171.8,171.7,171.5,165.6,165.3,165.2,165.2,165.1,165.1,133.5$, $133.5(\times 2), 133.4(\times 2), 132.6(\times 2), 132.5,130.1(\times 2), 130.0(\times 2), 130.0(\times 2), 129.9(\times 3), 129.9$ $(\times 3), 129.5,129.4(\times 2), 129.4,129.2,129.1,129.0(\times 2), 128.6(\times 3), 128.5(\times 4), 128.5(\times 4)$, 128.1, 100.4 (×2, $\left.\mathrm{Cl}^{\mathrm{B}}, \mathrm{C}^{\mathrm{C}}\right), 99.2\left(\mathrm{C1}^{\mathrm{D}}\right), 86.5\left(\mathrm{C1}^{\mathrm{A}}\right), 74.7\left(\mathrm{C} 4^{\mathrm{A}}\right), 74.5,74.3\left(\mathrm{C} 4^{\mathrm{B}}, \mathrm{C} 4^{\mathrm{C}}\right), 72.9$ $\left(\mathrm{C} 3^{\mathrm{A}}\right), 71.9\left(\mathrm{C}^{\mathrm{B}}\right), 71.6\left(\mathrm{C}^{\mathrm{C}}\right), 71.4\left(\mathrm{C}^{\mathrm{B}}\right), 71.1\left(\mathrm{C} 2^{\mathrm{C}}\right), 70.4\left(\mathrm{C}^{\mathrm{A}}\right), 70.2\left(\mathrm{C} 2^{\mathrm{D}}\right), 69.6\left(\mathrm{C} 3^{\mathrm{D}}\right), 68.7$ $\left(\mathrm{C} 4^{\mathrm{D}}\right), 66.0\left(\mathrm{C}^{\mathrm{A}}\right), 62.4\left(2 \mathrm{C}, \mathrm{C}^{\mathrm{B}}, \mathrm{C}^{\mathrm{C}}\right), 61.0\left(\mathrm{C}^{\mathrm{D}}\right), 37.8,37.8,37.8,29.8,29.8,29.6,28.0$, 28.0, 27.9. HRMS: $m / z$ calcd for $\mathrm{C}_{83} \mathrm{H}_{80} \mathrm{O}_{28} \mathrm{SNa}[\mathrm{M}+\mathrm{Na}]^{+} 1579.4449$, found 1579.4427 . 
Phenyl 2,3,4-tri-O-benzoyl- $\beta$-D-xylopyranosyl-(1 $(\rightarrow 4)$-2-O-benzoyl- $\beta$-D-xylopyranosyl-( $(\rightarrow 4)$-2-

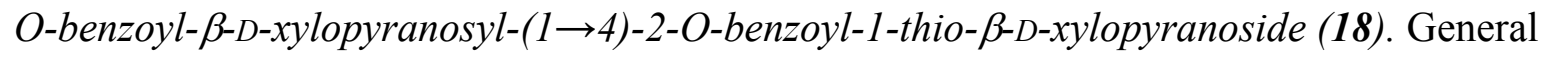
procedure $\mathrm{C}$ with tetrasaccharide $17(0.146 \mathrm{~g}, 0.094 \mathrm{mmol})$ and $50 \%$ solution of hydrazine hydrate $(59 \mu \mathrm{L}, 0.94 \mathrm{mmol})$. Reaction time $3 \mathrm{~h}$. Eluent for TLC (heptane/EtOAc 1:1). The product obtained was a white powder $(0.113 \mathrm{~g}, 96 \%) . R_{\mathrm{f}} 0.37$ (heptane/acetone $\left.1: 1\right) .[\alpha]^{25} \mathrm{D}$ $-36.6\left(c 1.0, \mathrm{CHCl}_{3}\right) .{ }^{1} \mathrm{H}$ NMR $\left(400 \mathrm{MHz}, \mathrm{CDCl}_{3}\right) \delta 8.06-8.00(\mathrm{~m}, 6 \mathrm{H}), 7.96-7.90(\mathrm{~m}, 6 \mathrm{H})$, 7.62-7.32 (m, 22H), 7.24-7.20 (m, 1H), $5.82\left(\mathrm{t}, J=8.9 \mathrm{~Hz}, 1 \mathrm{H}, \mathrm{H}^{\mathrm{D}}\right), 5.42\left(\mathrm{dd}, J_{2 \mathrm{D}, 3 \mathrm{D}}=9.0 \mathrm{~Hz}\right.$, $\left.J_{1 \mathrm{D}, 2 \mathrm{D}}=7.0 \mathrm{~Hz}, 1 \mathrm{H}, \mathrm{H} 1^{\mathrm{D}}\right), 5.37\left(\mathrm{td}, J_{3 \mathrm{D}, 4 \mathrm{D}}=8.9 \mathrm{~Hz}, J=4.5 \mathrm{~Hz}, 1 \mathrm{H}, \mathrm{H} 4 \mathrm{D}\right), 5.11-5.07(\mathrm{~m}, 1 \mathrm{H}$, $\left.\mathrm{H} 2^{\mathrm{C}}\right), 5.05-5.00\left(\mathrm{~m}, 2 \mathrm{H}, \mathrm{H} 2^{\mathrm{A}}, \mathrm{H} 2^{\mathrm{B}}\right), 4.83\left(\mathrm{~d}, J_{1 \mathrm{D}, 2 \mathrm{D}}=7.0 \mathrm{~Hz}, 1 \mathrm{H}, \mathrm{H} 1^{\mathrm{D}}\right), 4.64\left(\mathrm{~d}, J_{1 \mathrm{~A}, 2 \mathrm{~A}}=9.9 \mathrm{~Hz}\right.$, $\left.1 \mathrm{H}, \mathrm{H} 1^{\mathrm{A}}\right), 4.50\left(\mathrm{~d}, J_{1 \mathrm{C}, 2 \mathrm{C}}=7.9 \mathrm{~Hz}, 1 \mathrm{H}, \mathrm{H} 1^{\mathrm{C}}\right), 4.47-4.43\left(\mathrm{~m}, 2 \mathrm{H}, \mathrm{H} 1^{\mathrm{B}}, \mathrm{H} 5^{\mathrm{D}}\right), 3.87-3.77(\mathrm{~m}, 4 \mathrm{H}$, $\left.\mathrm{H}^{\mathrm{A}}, \mathrm{H} 3^{\mathrm{C}}, \mathrm{H} 4^{\mathrm{C}}, \mathrm{H} 5^{\mathrm{C}}\right), 3.75-3.55\left(\mathrm{~m}, 7 \mathrm{H}, \mathrm{H} 3^{\mathrm{A}}, \mathrm{H}^{\mathrm{A}}{ }^{\mathrm{A}}, \mathrm{H} 3^{\mathrm{B}}, \mathrm{H} 4^{\mathrm{B}}, \mathrm{H} 5^{\mathrm{B}}, \mathrm{H} 5^{\mathrm{D}}\right), 3.31-3.15$ (m, 3H, $\left.\mathrm{H} 5^{\circ}{ }^{\mathrm{A}}, \mathrm{H} 5^{\circ}{ }^{\mathrm{B}}, \mathrm{H} 5{ }^{\circ}\right) .{ }^{\mathrm{C}}{ }^{13} \mathrm{C}\left\{{ }^{1} \mathrm{H}\right\} \mathrm{NMR}\left(101 \mathrm{MHz}, \mathrm{CDCl}_{3}\right) \delta 165.6(\times 2), 165.5,165.5,165.4,165.2$, $133.8,133.7,133.6,133.5,133.5,133.3,132.8(\times 3), 132.3,130.0(\times 2), 129.9(\times 2), 129.9(\times 2)$, $129.9(\times 2), 129.8(\times 2), 129.7(\times 4), 129.5,129.4,129.1,129.0,129.0(\times 3), 128.9,128.9,128.9$, $128.8(\times 2), 128.7(\times 2), 128.6(\times 2), 128.6(\times 2), 128.5(\times 2), 128.5(\times 2), 128.3,128.1,102.2$, $102.1\left(\mathrm{Cl}^{\mathrm{B}}, \mathrm{Cl}^{\mathrm{C}}, J_{\mathrm{C}-\mathrm{H}}=157 \mathrm{~Hz}, J_{\mathrm{C}-\mathrm{H}}=163 \mathrm{~Hz}\right), 101.8\left(\mathrm{Cl}^{\mathrm{D}}, J_{\mathrm{C}-\mathrm{H}}=162 \mathrm{~Hz}\right), 86.6\left(\mathrm{C}^{\mathrm{A}}, J_{\mathrm{C}-\mathrm{H}}=\right.$ $155 \mathrm{~Hz}), 80.6\left(\mathrm{C}^{\mathrm{A}}\right), 80.3\left(\mathrm{C} 4^{\mathrm{B}}\right), 80.1\left(\mathrm{C} 4^{\mathrm{C}}\right), 74.8\left(\mathrm{C}^{\mathrm{A}}\right), 73.2,73.1,73.0,73.0\left(\mathrm{C}^{\mathrm{B}}, \mathrm{C}^{\mathrm{B}}, \mathrm{C}^{\mathrm{C}}\right.$, $\left.\mathrm{C}^{\mathrm{C}}\right), 72.0\left(\mathrm{C}^{\mathrm{A}}\right), 71.3\left(\mathrm{C}^{\mathrm{D}}\right), 71.1\left(\mathrm{C} 2^{\mathrm{D}}\right), 69.3\left(\mathrm{C} 4^{\mathrm{D}}\right), 67.1\left(\mathrm{C} 5^{\mathrm{A}}\right), 63.5\left(2 \mathrm{C}, \mathrm{C}^{\mathrm{B}}, \mathrm{C}^{\mathrm{C}}\right), 62.8$ (C5 ${ }^{\mathrm{D}}$ ). HRMS: $\mathrm{m} / z$ calcd for $\mathrm{C}_{68} \mathrm{H}_{62} \mathrm{O}_{22} \mathrm{SNa}[\mathrm{M}+\mathrm{Na}]^{+}$1285.3346, found 1285.3346 .

Phenyl 2,3,4-tri-O-benzoyl- $\beta$-D-xylopyranosyl-( $(1 \rightarrow 4)$-[2,3,5-tri-O-benzoyl- $\alpha$ - $L$ arabinofuranosyl-( $(1 \rightarrow 3)]-2-O$-benzoyl- $\beta$-D-xylopyranosyl- $(1 \rightarrow 4)$-[2,3,5-tri-O-benzoyl- $\alpha$-L- 
arabinofuranosyl-(l-3)]-2-O-benzoyl- $\beta$-D-xylopyranosyl-( $(1 \rightarrow 4)$-[2,3,5-tri-O-benzoyl- $\alpha$-Larabinofuranosyl-(1 $\rightarrow 3)$ ]-2-O-benzoyl-1-thio- $\beta$-D-xylopyranoside (19). General procedure D with acceptor $18(0.054 \mathrm{~g}, 0.043 \mathrm{mmol})$, donor $4(0.109 \mathrm{~g}, 0.17 \mathrm{mmol})$ and TMSOTf in dry $\mathrm{CH}_{2} \mathrm{Cl}_{2}(0.11 \mathrm{~mL}, c=0.04 \mathrm{mmol} / \mathrm{mL})$. Reaction time $75 \mathrm{~min}$ then $\mathrm{Et}_{3} \mathrm{~N}(0.02 \mathrm{~mL}, 0.11 \mathrm{mmol})$. Eluent TLC (heptane/acetone 1:1) and flash column chromatography (heptane/acetone 2:1). The product 19 was isolated as a white amorphous solid $(0.100 \mathrm{~g}, 90 \%) \cdot R_{\mathrm{f}} 0.41$ (heptane/acetone 1:1). $[\alpha]^{25} \mathrm{D}-46.9\left(c 1.0, \mathrm{CHCl}_{3}\right) .{ }^{1} \mathrm{H}$ NMR $\left(400 \mathrm{MHz}, \mathrm{CDCl}_{3}\right) \delta 8.18-8.16(\mathrm{~m}, 2 \mathrm{H}), 8.09-7.87$ (m, 28H), $7.83(\mathrm{t}, J=7.4 \mathrm{~Hz}, 1 \mathrm{H}), 7.64-7.16(\mathrm{~m}, 49 \mathrm{H}), 5.71\left(\mathrm{t}, J_{3 \mathrm{D}, 4 \mathrm{D}}=9.3 \mathrm{~Hz}, 1 \mathrm{H}, \mathrm{H} 3^{\mathrm{D}}\right), 5.52$ (d, $J=5.5 \mathrm{~Hz}, 1 \mathrm{H}), 5.49$ (d, $J=5.3 \mathrm{~Hz}, 1 \mathrm{H}), 5.45$ (d, $J=4.6 \mathrm{~Hz}, 1 \mathrm{H}), 5.43(\mathrm{~s}, 1 \mathrm{H}), 5.37$ (d, $J=$ $1.0 \mathrm{~Hz}, 1 \mathrm{H}), 5.35-5.34(\mathrm{~m}, 2 \mathrm{H}), 5.30-5.28\left(\mathrm{~m}, 2 \mathrm{H}, \mathrm{H} 2^{\mathrm{D}}\right), 5.26-5.24\left(\mathrm{~m}, 2 \mathrm{H}, \mathrm{H} 2^{\mathrm{A}}\right), 5.15(\mathrm{td}$, $\left.J_{3 \mathrm{D}, 4 \mathrm{D}}=9.3 \mathrm{~Hz}, J_{4 \mathrm{D}, 5 \mathrm{D}}=4.7 \mathrm{~Hz}, 1 \mathrm{H}, \mathrm{H} 4^{\mathrm{D}}\right), 5.07-4.83\left(\mathrm{~m}, 2 \mathrm{H}, \mathrm{H} 2^{\mathrm{B}}, \mathrm{H} 2^{\mathrm{C}}\right), 4.68(\mathrm{~d}, J=8.8 \mathrm{~Hz}$ $\left.1 \mathrm{H}, \mathrm{H} 1^{\mathrm{A}}\right), 4.23\left(\mathrm{~d}, J=8.0 \mathrm{~Hz}, 1 \mathrm{H}, \mathrm{H} 1^{\mathrm{B}}\right), 4.10\left(\mathrm{~d}, J=7.4 \mathrm{~Hz}, 1 \mathrm{H}, \mathrm{H} 1^{\mathrm{D}}\right), 4.08-3.99\left(\mathrm{~m}, 2 \mathrm{H}, \mathrm{H} 3^{\mathrm{A}}\right.$, $\left.\mathrm{H} 5^{\mathrm{A}}\right), 3.88-3.82\left(\mathrm{~m}, 3 \mathrm{H}, \mathrm{H} 3^{\mathrm{B}}, \mathrm{H}^{\mathrm{C}}, \mathrm{H} 5^{\mathrm{D}}\right), 3.76\left(\mathrm{td}, J=8.8,4.8 \mathrm{~Hz}, 1 \mathrm{H}, \mathrm{H} 4^{\mathrm{A}}\right), 3.59-3.53(\mathrm{~m}, 2 \mathrm{H}$, $\left.\mathrm{H}^{\mathrm{C}}, \mathrm{H} 4^{\mathrm{C}}\right), 3.33$ (dd, $\left.J=11.8,5.1 \mathrm{~Hz}, 1 \mathrm{H}, \mathrm{H} 5^{\mathrm{C}}\right), 3.29-3.19$ (m, 2H, H4 $\left.{ }^{\mathrm{B}}, \mathrm{H} 5^{\circ} \mathrm{D}\right), 3.10-3.02$ (m, $\left.2 \mathrm{H}, \mathrm{H} 5^{\circ}{ }^{\mathrm{A}}, \mathrm{H} 5^{\mathrm{B}}\right), 2.67-2.56\left(\mathrm{~m}, 2 \mathrm{H}, \mathrm{H} 5^{\circ}, \mathrm{H} 5^{\circ}\right) .{ }^{13} \mathrm{C}\left\{{ }^{1} \mathrm{H}\right\}$ NMR (101 MHz, $\left.\mathrm{CDCl}_{3}\right) \delta 166.4$, $166.4,166.4,166.1,165.9,165.8,165.7,165.1,165.1(\times 3), 165.0,164.7,164.4,164.2,133.9$, 133.7, 133.5, 133.4, 133.3, 133.2, 133.2, 133.1, 133.0, 132.9, 132.5, 130.4, 130.2, 130.2, 130.1, $130.1,130.0,129.9,129.9,129.9,129.9,129.8,129.8,129.7,129.7,129.7,129.6,129.5,129.3$, $129.2,129.1,129.1,129.0,129.0,129.0,129.0,128.9,128.9,128.6,128.6,128.5,128.5,128.4$, $128.4,128.3,128.3,128.2,127.9,106.1,105.7,105.6\left(C 1^{\mathrm{E}}, \mathrm{C} 1^{\mathrm{F}}, \mathrm{C} 1^{\mathrm{G}}\right), 100.3\left(\mathrm{C} 1^{\mathrm{B}}\right), 100.1$ $\left(\mathrm{C} 1^{\mathrm{C}}\right), 99.9\left(\mathrm{C1}^{\mathrm{B}}\right), 86.7\left(\mathrm{Cl}^{\mathrm{A}}\right), 82.8,82.7,82.5,82.1,81.5,80.8,78.3,78.2(\times 2), 76.0\left(\mathrm{C}^{\mathrm{A}}\right)$, $75.3\left(\mathrm{C}^{\mathrm{B}} / \mathrm{C} 3^{\mathrm{C}}\right), 75.2\left(\mathrm{C} 3^{\mathrm{B}} / \mathrm{C} 3^{\mathrm{C}}\right), 74.5\left(\mathrm{C} 4^{\mathrm{C}}\right), 74.2\left(\mathrm{C} 4^{\mathrm{B}}\right), 73.7\left(\mathrm{C} 4^{\mathrm{A}}\right), 73.3\left(\mathrm{C} 2^{\mathrm{B}}\right), 73.0\left(\mathrm{C} 2^{\mathrm{C}}\right)$, $72.2\left(\mathrm{C}^{\mathrm{A}} / \mathrm{C} 3^{\mathrm{D}}\right), 72.0\left(\mathrm{C} 2^{\mathrm{A}} / \mathrm{C}^{\mathrm{D}}\right), 71.2\left(\mathrm{C} 2^{\mathrm{D}}\right), 69.8\left(\mathrm{C}^{\mathrm{D}}\right), 66.1\left(\mathrm{C} 5^{\mathrm{A}}\right), 64.0,63.9,63.8\left(\mathrm{C} 5^{\mathrm{E}}, \mathrm{C}^{\mathrm{F}}\right.$, 
$\left.\mathrm{C}^{\mathrm{G}}\right), 63.1,63.1,63.0\left(\mathrm{C}^{\mathrm{B}}, \mathrm{C}^{\mathrm{C}}, \mathrm{C}^{\mathrm{D}}\right) . \mathrm{HRMS}: \mathrm{m} / z$ calcd for $\mathrm{C}_{146} \mathrm{H}_{122} \mathrm{O}_{43} \mathrm{SNa}\left[\mathrm{M}+\mathrm{Na}^{+}\right.$ 2617.6973, found 2617.6976.

Phenyl 2,3,4-tri-O-benzoyl- $\beta$-D-xylopyranosyl- $(1 \rightarrow 4)$-2-O-benzoyl-3-O-levulinoyl- $\beta$ - $D$ -

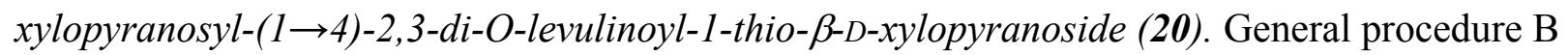
with crushed mol sieves (2.6 g), donor 14 (1.00 g, $1.12 \mathrm{mmol}), \operatorname{AgOTf}(0.579 \mathrm{~g}, 2.25 \mathrm{mmol})$ and $p \mathrm{NO}_{2} \mathrm{PhSCl}(0.214 \mathrm{~g}, 1.12 \mathrm{mmol})$. Activation time $40 \mathrm{~min}$ then acceptor $9(0.447 \mathrm{~g}, 1.02$ mmol). Reaction time 50 min then $\mathrm{Et}_{3} \mathrm{~N}(0.47 \mathrm{~mL}, 3.37 \mathrm{mmol})$. Purification by flash column chromatography (hexane/toluene/EtOAc 3:1:4) gave 20 as a white amorphous solid (0.883 g, 71\%). In addition, donor 14 was reisolated $(0.119 \mathrm{~g}, 12 \%) . R_{\mathrm{f}} 0.48$ (hexane/acetone $\left.3: 2\right)$. $[\alpha]^{25} \mathrm{D}$ $-60.8\left(c\right.$ 1.0, $\left.\mathrm{CHCl}_{3}\right) .{ }^{1} \mathrm{H}$ NMR $\left(400 \mathrm{MHz}, \mathrm{CDCl}_{3}\right) \delta 8.02-7.92(\mathrm{~m}, 8 \mathrm{H}), 7.61-7.43(\mathrm{~m}, 6 \mathrm{H})$, $7.41-7.32(\mathrm{~m}, 8 \mathrm{H}), 7.28-7.26(\mathrm{~m}, 3 \mathrm{H}), 5.70\left(\mathrm{t}, J=6.5 \mathrm{~Hz}, 1 \mathrm{H}, \mathrm{H} 3^{\mathrm{C}}\right), 5.31(\mathrm{t}, J=8.1 \mathrm{~Hz}, 1 \mathrm{H}$, $\left.\mathrm{H}^{\mathrm{B}}\right), 5.28-5.24\left(\mathrm{~m}, 2 \mathrm{H}, \mathrm{H}^{\mathrm{C}}, \mathrm{H}^{\mathrm{C}}\right), 5.11\left(\mathrm{t}, J_{2 \mathrm{~A}, 3 \mathrm{~A}}=8.7 \mathrm{~Hz}, 1 \mathrm{H}, \mathrm{H} 3^{\mathrm{A}}\right), 5.00\left(\mathrm{dd}, J_{2 \mathrm{~B}, 3 \mathrm{~B}}=8.3 \mathrm{~Hz}\right.$, $\left.J_{1 \mathrm{~B}, 2 \mathrm{~B}}=6.4 \mathrm{~Hz}, 1 \mathrm{H}, \mathrm{H} 2^{\mathrm{B}}\right), 4.97\left(\mathrm{~d}, J_{1 \mathrm{C}, 2 \mathrm{C}}=4.7 \mathrm{~Hz}, 1 \mathrm{H}, \mathrm{H} 1^{\mathrm{C}}\right), 4.84\left(\mathrm{t}, J_{2 \mathrm{~A}, 3 \mathrm{~A}}=9.0 \mathrm{~Hz}, 1 \mathrm{H}, \mathrm{H} 2^{\mathrm{A}}\right)$, $4.61-4.58\left(\mathrm{~m}, 2 \mathrm{H}, \mathrm{H} 1^{\mathrm{A}}, \mathrm{H}^{\mathrm{B}}\right), 4.46\left(\mathrm{dd}, J_{5 \mathrm{Ceq}, 5 \mathrm{Cax}}=12.4 \mathrm{~Hz}, J_{5 \mathrm{Ceq}, 4 \mathrm{C}}=3.8 \mathrm{~Hz}, 1 \mathrm{H}, \mathrm{H}^{\mathrm{C}}{ }_{\mathrm{eq}}\right), 4.06$ $\left(\mathrm{dd}, J_{5 \mathrm{Beq}, 5 \mathrm{Bax}}=11.9 \mathrm{~Hz}, J_{5 \mathrm{Beq}, 4 \mathrm{~B}}=4.8 \mathrm{~Hz}, 1 \mathrm{H}, \mathrm{H}^{\mathrm{B}}{ }_{\mathrm{eq}}\right), 4.02-3.98\left(\mathrm{~m}, 1 \mathrm{H}, \mathrm{H} 4^{\mathrm{B}}\right), 3.94(\mathrm{dd}$, $\left.J_{5 \text { Aeq }, 5 \text { Aax }}=11.9 \mathrm{~Hz}, J_{5 \text { Aeq }, 4 \mathrm{~A}}=5.2 \mathrm{~Hz}, 1 \mathrm{H}, \mathrm{H}^{\mathrm{A}}{ }_{\text {eq }}\right), 3.77-3.71\left(\mathrm{~m}, 2 \mathrm{H}, \mathrm{H}^{\mathrm{A}}{ }^{\mathrm{A}}, \mathrm{H}^{\mathrm{c}}{ }_{\mathrm{ax}}\right), 3.38(\mathrm{dd}$, $\left.J_{5 \mathrm{Bax}, 5 \mathrm{Beq}}=11.9 \mathrm{~Hz}, J_{5 \mathrm{Bax}, 4 \mathrm{~B}}=8.1 \mathrm{~Hz}, 1 \mathrm{H}, \mathrm{H}^{\mathrm{B}}{ }_{\mathrm{ax}}\right), 3.21\left(\mathrm{dd}, J_{5 \mathrm{Aax}, 5 \mathrm{Aeq}}=11.8 \mathrm{~Hz}, J_{5 \mathrm{Aax}, 4 \mathrm{~A}}=9.8\right.$ $\left.\mathrm{Hz}, 1 \mathrm{H}, \mathrm{H} 5^{\mathrm{A}}{ }_{\mathrm{ax}}\right), 2.85-2.39(\mathrm{~m}, 12 \mathrm{H}), 2.17(\mathrm{~s}, 3 \mathrm{H}), 2.16(\mathrm{~s}, 3 \mathrm{H}), 1.98(\mathrm{~s}, 3 \mathrm{H}) .{ }^{13} \mathrm{C}\left\{{ }^{1} \mathrm{H}\right\} \mathrm{NMR}$ $\left(101 \mathrm{MHz}, \mathrm{CDCl}_{3}\right) \delta 206.5,206.4,206.0,171.9,171.7,171.5,165.6,165.4,165.2,165.1,133.5$, $133.5,133.5,133.5,132.8(\times 2), 132.3,130.1(\times 2), 130.0(\times 2), 130.0(\times 2), 129.9(\times 2), 129.4$, 129.4, 129.3, 129.2, $129.1(\times 2), 128.7(\times 2), 128.5(\times 4), 128.2,100.6\left(\mathrm{C} 1^{\mathrm{B}}\right), 99.3\left(\mathrm{C} 1^{\mathrm{C}}\right), 86.5$ $\left(\mathrm{C} 1^{\mathrm{A}}\right), 75.2\left(\mathrm{C} 4^{\mathrm{A}}\right), 74.3\left(\mathrm{C}^{\mathrm{B}}\right), 73.4\left(\mathrm{C} 3^{\mathrm{A}}\right), 71.9\left(\mathrm{C} 3^{\mathrm{B}}\right), 71.4\left(\mathrm{C} 2^{\mathrm{B}}\right), 70.2,70.0\left(\mathrm{C} 2^{\mathrm{A}}, \mathrm{C} 2^{\mathrm{C}}\right), 69.7$ 
$\left(\mathrm{C}^{\mathrm{C}}\right), 68.8\left(\mathrm{C}^{\mathrm{C}}\right), 66.6\left(\mathrm{C}^{\mathrm{A}}\right), 62.5\left(\mathrm{C}^{\mathrm{B}}\right), 61.1\left(\mathrm{C}^{\mathrm{C}}\right), 37.9,37.9,37.8,30.0,29.9,29.7,28.1$, 28.0, 28.0. HRMS: $m / z$ calcd for $\mathrm{C}_{64} \mathrm{H}_{64} \mathrm{O}_{22} \mathrm{SNa}[\mathrm{M}+\mathrm{Na}]^{+}$1239.3502, found 1239.3489.

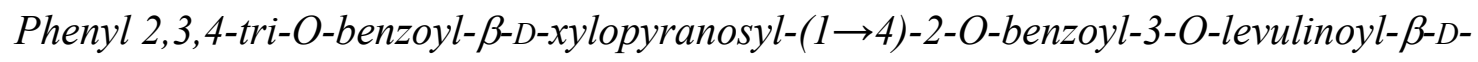

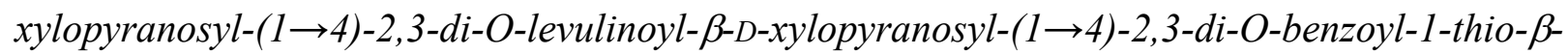
D-xylopyranoside (21). General procedure B with crushed mol sieves $(2.1 \mathrm{~g})$, donor 20 (1.21 g, $0.99 \mathrm{mmol}), \operatorname{AgOTf}(0.510 \mathrm{~g}, 1.98 \mathrm{mmol}), p \mathrm{NO}_{2} \mathrm{PhSCl}(0.187 \mathrm{~g}, 0.99 \mathrm{mmol})$ and acceptor 6 (0.402 g, $0.89 \mathrm{mmol})$. Reaction time 40 min then $\mathrm{Et}_{3} \mathrm{~N}(0.40 \mathrm{~mL}, 2.97 \mathrm{mmol})$. Purification by flash column chromatography (heptane/toluene/acetone 3:2:2) gave 21 as a white amorphous solid (0.851 g, 61\%). $R_{\mathrm{f}} 0.40$ (hexane/acetone 3:2). [ $\left.\alpha\right]^{25} \mathrm{D}-38.2\left(c\right.$ 1.0, $\left.\mathrm{CHCl}_{3}\right) .{ }^{1} \mathrm{H}$ NMR (400 $\left.\mathrm{MHz}, \mathrm{CDCl}_{3}\right) \delta 8.01-7.91(\mathrm{~m}, 12 \mathrm{H}), 7.61(\mathrm{t}, J=7.4 \mathrm{~Hz}, 1 \mathrm{H}), 7.56-7.45(\mathrm{~m}, 9 \mathrm{H}), 7.41-7.32(\mathrm{~m}$, $10 \mathrm{H}), 7.29-7.24(\mathrm{~m}, 2 \mathrm{H}), 7.18-7.16(\mathrm{~m}, 1 \mathrm{H}), 5.70\left(\mathrm{t}, J=6.5 \mathrm{~Hz}, 1 \mathrm{H}, \mathrm{H} 3^{\mathrm{D}}\right), 5.53(\mathrm{t}, J=7.6 \mathrm{~Hz}$, $\left.1 \mathrm{H}, \mathrm{H} 3^{\mathrm{A}}\right), 5.30-5.24\left(\mathrm{~m}, 4 \mathrm{H}, \mathrm{H} 2^{\mathrm{A}}, \mathrm{H} 3^{\mathrm{C}}, \mathrm{H} 2^{\mathrm{D}}, \mathrm{H} 4^{\mathrm{D}}\right), 5.03\left(\mathrm{~d}, J_{1 \mathrm{~A}, 2 \mathrm{~A}}=7.7 \mathrm{~Hz}, 1 \mathrm{H}, \mathrm{H}^{\mathrm{A}}\right)$, $4.99-4.91\left(\mathrm{~m}, 3 \mathrm{H}, \mathrm{H} 3^{\mathrm{B}}, \mathrm{H} 2^{\mathrm{C}}, \mathrm{H} 1^{\mathrm{D}}\right), 4.70\left(\mathrm{dd}, J_{2 \mathrm{~B}, 3 \mathrm{~B}}=8.9 \mathrm{~Hz}, J_{1 \mathrm{~B}, 2 \mathrm{~B}}=7.0 \mathrm{~Hz}, 1 \mathrm{H}, \mathrm{H} 2^{\mathrm{B}}\right), 4.45$ $\left(\mathrm{dd}, J_{5 \mathrm{Deq}, 5 \mathrm{Dax}}=12.4 \mathrm{~Hz}, J_{5 \mathrm{Deq}, 4 \mathrm{D}}=3.8 \mathrm{~Hz}, 1 \mathrm{H}, \mathrm{H}^{\mathrm{D}}{ }_{\text {eq }}\right), 4.41\left(\mathrm{~d}, J_{1 \mathrm{~B}, 2 \mathrm{~B}}=6.9 \mathrm{~Hz}, 1 \mathrm{H}, \mathrm{H} 1^{\mathrm{B}}\right), 4.36$ $\left(\mathrm{d}, J_{1 \mathrm{C}, 2 \mathrm{C}}=6.5 \mathrm{~Hz}, 1 \mathrm{H}, \mathrm{H}^{\mathrm{C}}\right), 4.30\left(\mathrm{dd}, J_{5 \mathrm{Aeq}, 5 \mathrm{Aax}}=12.1 \mathrm{~Hz}, J_{5 \mathrm{Aeq}, 4 \mathrm{~A}}=4.6 \mathrm{~Hz}, 1 \mathrm{H}, \mathrm{H} 5^{\mathrm{A}}{ }_{\mathrm{eq}}\right)$, 4.01-3.86 (m, 3H, H4 $\left.{ }^{\mathrm{A}}, \mathrm{H}^{\mathrm{C}}{ }^{\mathrm{C}}, \mathrm{H} 5^{\mathrm{C}}\right), 3.74\left(\mathrm{dd}, J_{5 \mathrm{Dax}, 5 \mathrm{Deq}}=12.4 \mathrm{~Hz}, J_{5 \mathrm{Dax}, 4 \mathrm{D}}=6.1 \mathrm{~Hz}, 1 \mathrm{H}, \mathrm{H}^{\mathrm{D}}{ }_{\mathrm{ax}}\right)$, $3.58\left(\mathrm{dd}, J_{5 \mathrm{Aax}, 5 \mathrm{Aeq}}=12.1 \mathrm{~Hz}, J_{5 \mathrm{Aax}, 4 \mathrm{~A}}=8.2 \mathrm{~Hz}, 1 \mathrm{H}, \mathrm{H}^{\mathrm{A}}{ }_{\mathrm{ax}}\right), 3.49-3.44\left(\mathrm{~m}, 1 \mathrm{H}, \mathrm{H} 4^{\mathrm{B}}\right), 3.34-3.28$ $\left(\mathrm{m}, 2 \mathrm{H}, \mathrm{H}^{\mathrm{B}}{ }_{\text {eq }}, \mathrm{H}^{\mathrm{C}}\right), 2.89\left(\mathrm{dd}, J_{5 \mathrm{Bax}, 5 \mathrm{Beq}}=12.0 \mathrm{~Hz}, J_{5 \mathrm{Bax}, 4 \mathrm{~B}}=9.4 \mathrm{~Hz}, 1 \mathrm{H}, \mathrm{H} 5^{\mathrm{B}}{ }_{\text {ax }}\right), 2.71-2.35(\mathrm{~m}$, 12H), 2.14 (bs, 6H), 1.97 (s, 3H). ${ }^{13} \mathrm{C}\left\{{ }^{1} \mathrm{H}\right\}$ NMR (101 MHz, $\left.\mathrm{CDCl}_{3}\right) \delta 206.4$, 206.4, 205.9, $171.8,171.7,171.3,165.6,165.4,165.3(\times 2), 165.2,165.0,133.5,133.5,133.5,133.5,133.3$, $133.2,133.0,132.6(\times 2), 130.1(\times 3), 130.0(\times 2), 130.0(\times 2), 129.9(\times 2), 129.8(\times 2), 129.6$, $129.5,129.4,129.4,129.2,129.2,129.1,129.1(\times 2), 128.6(\times 2), 128.6(\times 3), 128.5(\times 4), 128.5$ 
$(\times 2), 128.4(\times 2), 128.3,128.1,100.9\left(\mathrm{C1}^{\mathrm{B}}\right), 100.3\left(\mathrm{C1}^{\mathrm{C}}\right), 99.2\left(\mathrm{C1}^{\mathrm{D}}\right), 86.6\left(\mathrm{C} 1^{\mathrm{A}}\right), 75.4\left(\mathrm{C} 4^{\mathrm{A}}\right)$, $74.8\left(\mathrm{C}^{\mathrm{B}}\right), 74.4\left(\mathrm{C} 4^{\mathrm{C}}\right), 72.7\left(\mathrm{C}^{\mathrm{A}}\right), 72.1,71.9\left(\mathrm{C} 3^{\mathrm{B}}, \mathrm{C} 2^{\mathrm{A}} / \mathrm{C} 3^{\mathrm{C}} / \mathrm{C} 2^{\mathrm{D}}\right), 71.3,71.2\left(\mathrm{C} 2^{\mathrm{B}}, \mathrm{C} 2^{\mathrm{C}}\right), 70.4$, $70.2\left(\mathrm{C} 2^{\mathrm{A}} / \mathrm{C} 3^{\mathrm{C}} / \mathrm{C} 2^{\mathrm{D}}\right), 69.6\left(\mathrm{C} 3^{\mathrm{D}}\right), 68.7\left(\mathrm{C}^{\mathrm{D}}\right), 65.5\left(\mathrm{C} 5^{\mathrm{A}}\right), 62.6,62.5\left(\mathrm{C}^{\mathrm{B}}, \mathrm{C}^{\mathrm{C}}\right), 61.1\left(\mathrm{C} 5^{\mathrm{D}}\right)$, $37.8,37.8,37.7,30.0,29.9,29.6,28.0,27.9,27.9$. HRMS: $m / z$ calcd for $\mathrm{C}_{83} \mathrm{H}_{80} \mathrm{O}_{28} \mathrm{SNa}[\mathrm{M}+$ $\mathrm{Na}]^{+}$1579.4449, found 1579.4430 .

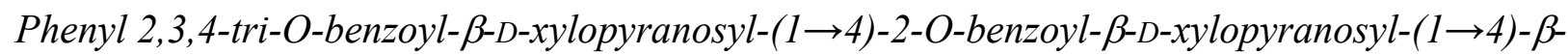
D-xylopyranosyl-(1 $\rightarrow 4)$-2,3-di-O-benzoyl-1-thio- $\beta$-D-xylopyranoside (22). General procedure C with tetrasaccharide $21(0.507 \mathrm{~g}, 0.33 \mathrm{mmol})$ and 50\% solution of hydrazine hydrate $(0.20 \mathrm{~mL}$, $3.21 \mathrm{mmol}$ ). Reaction time $20 \mathrm{~min}$. Eluent for TLC (hexane/toluene/acetone 2:1:2). The product was a white powder $(0.400 \mathrm{~g}, 97 \%) . R_{\mathrm{f}} 0.24$ (hexane/acetone $\left.3: 2\right)$. $[\alpha]^{25} \mathrm{D}-15.4\left(c 1.0, \mathrm{CHCl}_{3}\right)$. ${ }^{1} \mathrm{H}$ NMR $\left(400 \mathrm{MHz}, \mathrm{CDCl}_{3}\right) \delta 8.04-7.92(\mathrm{~m}, 12 \mathrm{H}), 7.63(\mathrm{t}, J=7.4 \mathrm{~Hz}, 1 \mathrm{H}), 7.57-7.26(\mathrm{~m}$, $22 \mathrm{H}), 5.84\left(\mathrm{t}, J_{2 \mathrm{D}, 3 \mathrm{D}}=8.8 \mathrm{~Hz}, 1 \mathrm{H}, \mathrm{H}^{\mathrm{D}}\right), 5.57\left(\mathrm{t}, J_{2 \mathrm{~A}, 3 \mathrm{~A}}=7.5 \mathrm{~Hz}, 1 \mathrm{H}, \mathrm{H} 3^{\mathrm{A}}\right), 5.43\left(\mathrm{dd}, J_{2 \mathrm{D}, 3 \mathrm{D}}=8.7\right.$ $\left.\mathrm{Hz}, J_{1 \mathrm{D}, 2 \mathrm{D}}=7.1 \mathrm{~Hz}, 1 \mathrm{H}, \mathrm{H} 2^{\mathrm{D}}\right), 5.38-5.30\left(\mathrm{~m}, 2 \mathrm{H}, \mathrm{H} 2^{\mathrm{A}}, \mathrm{H}^{\mathrm{D}}\right), 5.10\left(\mathrm{~d}, J_{1 \mathrm{~A}, 2 \mathrm{~A}}=7.4 \mathrm{~Hz}, 1 \mathrm{H}, \mathrm{H} 1^{\mathrm{A}}\right)$, $5.05\left(\mathrm{t}, J=8.2 \mathrm{~Hz}, 1 \mathrm{H}, \mathrm{H} 2^{\mathrm{C}}\right), 4.88\left(\mathrm{~d}, J_{1 \mathrm{D}, 2 \mathrm{D}}=6.9 \mathrm{~Hz}, 1 \mathrm{H}, \mathrm{H} 1^{\mathrm{D}}\right), 4.42-4.36\left(\mathrm{~m}, 2 \mathrm{H}, \mathrm{H} 5^{\mathrm{A}}, \mathrm{H} 5^{\mathrm{D}}\right)$, $4.33\left(\mathrm{~d}, J=7.6 \mathrm{~Hz}, 1 \mathrm{H}, \mathrm{H}^{\mathrm{C}}\right), 4.27\left(\mathrm{~d}, J=6.7 \mathrm{~Hz}, 1 \mathrm{H}, \mathrm{H} 1^{\mathrm{B}}\right), 3.97-3.92\left(\mathrm{~m}, 1 \mathrm{H}, \mathrm{H} 4^{\mathrm{A}}\right), 3.89-3.81$ (m, 3H, H3 $\left.{ }^{\mathrm{C}}, \mathrm{H}^{\mathrm{C}}{ }^{\mathrm{H}}, \mathrm{H}^{\mathrm{C}}\right), 3.73-3.62\left(\mathrm{~m}, 4 \mathrm{H}, \mathrm{H} 5^{\mathrm{A}}{ }^{\mathrm{A}}, \mathrm{H} 5^{\circ} \mathrm{D}, \mathrm{OH}\right), 3.45\left(\mathrm{t}, J=8.1 \mathrm{~Hz}, 1 \mathrm{H}, \mathrm{H} 3^{\mathrm{B}}\right)$, 3.33-3.19 (m, 4H, H2 $\left.{ }^{\mathrm{B}}, \mathrm{H}^{\mathrm{B}}{ }^{\mathrm{H}}, \mathrm{H}^{\mathrm{B}}{ }_{\mathrm{eq}}, \mathrm{H} 5^{\circ} \mathrm{C}\right), 3.05(\mathrm{bs}, 1 \mathrm{H}, \mathrm{OH}) 2.89\left(\mathrm{dd}, J_{5 \mathrm{Bax}, 5 \mathrm{Beq}}=11.5 \mathrm{~Hz}\right.$ $\left.J_{5 \text { bax }, 4 \mathrm{~B}}=9.2 \mathrm{~Hz}, 1 \mathrm{H}, \mathrm{H}^{\mathrm{B}}{ }_{\mathrm{ax}}\right) .{ }^{13} \mathrm{C}\left\{{ }^{1} \mathrm{H}\right\} \mathrm{NMR}\left(101 \mathrm{MHz}, \mathrm{CDCl}_{3}\right) \delta 165.6,165.6,165.6,165.6$, $165.3,165.2,133.8,133.7,133.5(\times 2), 133.4,133.3,133.0,132.6(\times 3), 130.0(\times 4), 129.9(\times 4)$, $129.9(\times 2), 129.8(\times 2), 129.6,129.5,129.5,129.1(\times 2), 129.0,129.0,128.9,128.7(\times 2), 128.7$ $(\times 2), 128.6(\times 2), 128.5(\times 2), 128.5(\times 2), 128.4(\times 2), 128.2,102.1\left(\mathrm{C}^{\mathrm{B}}, J_{\mathrm{C}-\mathrm{H}}=160 \mathrm{~Hz}\right), 101.8$ $\left(\mathrm{C} 1^{\mathrm{D}}, J_{\mathrm{C}-\mathrm{H}}=163 \mathrm{~Hz}\right), 101.4\left(\mathrm{C}^{\mathrm{C}}, J_{\mathrm{C}-\mathrm{H}}=163 \mathrm{~Hz}\right), 86.6\left(\mathrm{C}^{\mathrm{A}}, J_{\mathrm{C}-\mathrm{H}}=159 \mathrm{~Hz}\right), 80.2\left(\mathrm{C}^{\mathrm{C}}\right), 79.0$ 
$\left(\mathrm{C} 4^{\mathrm{B}}\right), 74.1\left(\mathrm{C} 4^{\mathrm{A}}\right), 73.3,73.1,73.0\left(\mathrm{C}^{\mathrm{B}}, \mathrm{C}^{\mathrm{C}}, \mathrm{C}^{\mathrm{C}}\right), 72.5,72.3\left(\mathrm{C}^{\mathrm{A}}, \mathrm{C}^{\mathrm{B}}\right), 71.4,71.2\left(\mathrm{C} 2^{\mathrm{D}}\right.$, C3 $\left.3^{\mathrm{D}}\right), 70.2\left(\mathrm{C}^{\mathrm{A}}\right), 69.4\left(\mathrm{C}^{\mathrm{D}}\right), 65.1\left(\mathrm{C}^{\mathrm{a}}\right), 63.4\left(\mathrm{C} 5^{\mathrm{C}}\right), 62.8\left(\mathrm{C}^{\mathrm{D}}\right), 62.0\left(\mathrm{C} 5^{\mathrm{B}}\right)$. HRMS: $\mathrm{m} / z$ calcd for $\mathrm{C}_{68} \mathrm{H}_{62} \mathrm{O}_{22} \mathrm{SNa}[\mathrm{M}+\mathrm{Na}]^{+}$1285.3345, found 1285.3334 .

Phenyl 2,3,4-tri-O-benzoyl- $\beta$-D-xylopyranosyl-( $(1 \rightarrow 4)$-[2,3,5-tri-O-benzoyl- $\alpha$-L-

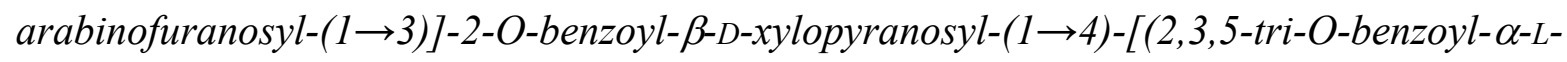
arabinofuranosyl-( $(1 \rightarrow 2)]$-[2,3,5-tri-O-benzoyl- $\alpha$-L-arabinofuranosyl-( $(1 \rightarrow 3)]$ - $\beta$-Dxylopyranosyl-(1 $\rightarrow 4$ )-2,3-di-O-benzoyl-1-thio- $\beta$-D-xylopyranoside (23). General procedure D with acceptor $22(0.201 \mathrm{~g}, 0.16 \mathrm{mmol})$, donor $4(0.401 \mathrm{~g}, 0.63 \mathrm{mmol})$ and TMSOTf in dry $\mathrm{CH}_{2} \mathrm{Cl}_{2}(0.16 \mathrm{~mL}, c=0.10 \mathrm{mmol} / \mathrm{mL})$. Reaction time $1 \mathrm{~h}$ then $\mathrm{Et}_{3} \mathrm{~N}(0.02 \mathrm{~mL}, 0.11 \mathrm{mmol})$. Eluent TLC analysis (hexane/toluene/acetone 2:1:1) and eluent flash column chromatography (hexane/toluene/acetone $3: 2: 1 \rightarrow 2: 1: 1)$. The product was a white amorphous solid $(0.393 \mathrm{~g}$, 95\%). $R_{\mathrm{f}} 0.42$ (hexane/toluene/acetone 2:1:1). $[\alpha]^{25} \mathrm{D}-52.5\left(c 1.0, \mathrm{CHCl}_{3}\right) .{ }^{1} \mathrm{H}$ NMR $(400 \mathrm{MHz}$, $\left.\mathrm{CDCl}_{3}\right) \delta 8.18(\mathrm{~d}, J=7.3 \mathrm{~Hz}, 2 \mathrm{H}), 8.09-7.73(\mathrm{~m}, 28 \mathrm{H}), 7.65(\mathrm{~d}, J=7.3 \mathrm{~Hz}, 2 \mathrm{H}), 7.58-7.05(\mathrm{~m}$, $48 \mathrm{H}), 5.76\left(\mathrm{~s}, 1 \mathrm{H}, \mathrm{H} 1^{\mathrm{F}}\right), 5.69\left(\mathrm{t}, J=9.1 \mathrm{~Hz}, 1 \mathrm{H}, \mathrm{H} 3^{\mathrm{D}}\right), 5.65\left(\mathrm{~s}, 1 \mathrm{H}, \mathrm{H} 1^{\mathrm{E}}\right), 5.54-5.47(\mathrm{~m}, 6 \mathrm{H}$, $\left.\mathrm{H}^{\mathrm{A}}\right), 5.34-5.34\left(\mathrm{~m}, 1 \mathrm{H}, \mathrm{H} 2^{\mathrm{E}}\right), 5.25\left(\mathrm{~s}, 1 \mathrm{H}, \mathrm{H} 1^{\mathrm{G}}\right), 5.25-5.20\left(\mathrm{~m}, 2 \mathrm{H}, \mathrm{H} 2^{\mathrm{A}}, \mathrm{H} 2^{\mathrm{D}}\right), 5.19-5.13(\mathrm{~m}$, $\left.1 \mathrm{H}, \mathrm{H} 4^{\mathrm{D}}\right), 5.10-5.06(\mathrm{~m}, 2 \mathrm{H}), 5.04-4.96\left(\mathrm{~m}, 3 \mathrm{H}, \mathrm{H} 2^{\mathrm{C}}\right), 4.94-4.85\left(\mathrm{~m}, 3 \mathrm{H}, \mathrm{H} 1^{\mathrm{A}}\right), 4.79(\mathrm{dd}, J=$ 11.4, 3.0 Hz, 1H), 4.71-4.62 (m, 2H), $4.31\left(\mathrm{~d}, J_{1 \mathrm{~B}, 2 \mathrm{~B}}=6.9 \mathrm{~Hz}, 1 \mathrm{H}, \mathrm{H} 1^{\mathrm{B}}\right), 4.17-4.13(\mathrm{~m}, 2 \mathrm{H}$, $\left.\mathrm{H}^{\mathrm{A}}, \mathrm{H} 1^{\mathrm{D}}\right), 3.88-3.81\left(\mathrm{~m}, 4 \mathrm{H}, \mathrm{H}^{\mathrm{B}}, \mathrm{H}^{\mathrm{C}}, \mathrm{H}^{\mathrm{C}}, \mathrm{H} 5^{\mathrm{D}}\right), 3.79-3.74\left(\mathrm{~m}, 1 \mathrm{H}, \mathrm{H} 4^{\mathrm{A}}\right), 3.65\left(\mathrm{dd}, J_{2 \mathrm{~B}, 3 \mathrm{~B}}=\right.$ $\left.9.5 \mathrm{~Hz}, J_{1 \mathrm{~B}, 2 \mathrm{~B}}=7.0 \mathrm{~Hz}, 1 \mathrm{H}, \mathrm{H} 2^{\mathrm{B}}\right), 3.53-3.47\left(\mathrm{~m}, 2 \mathrm{H}, \mathrm{H} 5^{\circ}{ }^{\mathrm{A}}, \mathrm{H} 4^{\mathrm{C}}\right), 3.32-3.26\left(\mathrm{~m}, 3 \mathrm{H}, \mathrm{H} 4^{\mathrm{B}}, \mathrm{H}^{\mathrm{B}}\right.$, $\left.\mathrm{H}^{\prime}{ }^{\mathrm{D}}\right), 3.03\left(\mathrm{dd}, J_{5 \mathrm{C}, 5 \mathrm{C}^{\prime}}=11.8 \mathrm{~Hz}, J_{5 \mathrm{C}, 4 \mathrm{C}}=5.1 \mathrm{~Hz}, 1 \mathrm{H}, \mathrm{H} 5^{\mathrm{C}}\right), 2.73-2.67\left(\mathrm{~m}, 1 \mathrm{H}, \mathrm{H} 5^{\circ} \mathrm{B}\right), 2.53(\mathrm{t}, J$ $\left.=11.1 \mathrm{~Hz}, 1 \mathrm{H}, \mathrm{H} 5^{\circ}\right) .{ }^{13} \mathrm{C}\left\{{ }^{1} \mathrm{H}\right\} \mathrm{NMR}\left(101 \mathrm{MHz}, \mathrm{CDCl}_{3}\right) \delta 166.5,166.3,166.3,166.0,165.8$, $165.8,165.7,165.6,165.3,165.2(\times 2), 165.0,165.0,164.5,164.2,133.6,133.6,133.6,133.5$, 
133.4, 133.4, 133.3, 133.2, 133.1, 133.0, 132.9, 132.8, 132.2, 130.3, 130.1, 130.1, 130.0, 129.9, $129.9,129.8,129.8,129.7,129.7,129.5,129.2,129.1,129.1,129.0,129.0,129.0,128.9,128.9$, 128.6, 128.6, 128.4, 128.4, 128.3, 128.3, 128.2, 127.9, $106.9\left(\mathrm{C1}^{\mathrm{E}}\right), 106.2\left(\mathrm{C1}^{\mathrm{G}}\right), 105.4\left(\mathrm{C1}^{\mathrm{F}}\right)$, $101.7\left(\mathrm{Cl}^{\mathrm{B}}\right), 99.8\left(\mathrm{C1}^{\mathrm{D}}\right), 99.5\left(\mathrm{C} 1^{\mathrm{C}}\right), 86.2\left(\mathrm{C} 1^{\mathrm{A}}\right), 82.6\left(\mathrm{C} 2^{\mathrm{F}}\right), 82.3,82.2,81.8,81.8,80.7,79.7$ $\left(\mathrm{C} 2^{\mathrm{B}}\right), 78.6,78.1,77.8,76.0\left(\mathrm{C}^{\mathrm{B}} / \mathrm{C}^{\mathrm{C}}\right), 75.8\left(\mathrm{C} 3^{\mathrm{B}} / \mathrm{C}^{\mathrm{C}}\right), 74.6\left(\mathrm{C} 4^{\mathrm{C}}\right), 73.8\left(\mathrm{C} 4^{\mathrm{B}}\right), 73.6\left(\mathrm{C}^{\mathrm{A}}\right)$, $73.1\left(\mathrm{C}^{\mathrm{C}}\right), 71.9\left(\mathrm{C}^{\mathrm{D}}\right), 71.8\left(\mathrm{C} 3^{\mathrm{A}}\right), 71.1\left(\mathrm{C}^{\mathrm{D}}\right), 70.2\left(\mathrm{C} 2^{\mathrm{A}}\right), 69.7\left(\mathrm{C} 4^{\mathrm{D}}\right), 64.6\left(\mathrm{C} 5^{\mathrm{A}}\right)$, 64.2, 64.0, $63.9\left(\mathrm{C}^{\mathrm{E}}, \mathrm{C}^{\mathrm{F}}, \mathrm{C} 5^{\mathrm{G}}\right), 63.0\left(\mathrm{C} 5^{\mathrm{C}} / \mathrm{C}^{\mathrm{D}}\right), 62.8\left(\mathrm{C} 5^{\mathrm{C}} / \mathrm{C}^{\mathrm{D}}\right), 62.3\left(\mathrm{C}^{\mathrm{B}}\right)$. HRMS: $m / z$ calcd for $\mathrm{C}_{146} \mathrm{H}_{122} \mathrm{O}_{43} \mathrm{~S}\left(\mathrm{Na}^{+}\right)_{2}[\mathrm{M}+2 \mathrm{Na}]^{2+}$ 1320.3432; found $m / z$ 1320.3463.

Phenyl 2,3,4-tri-O-benzoyl- $\beta$-D-xylopyranosyl-(1 $1 \rightarrow 4)$-2,3-di-O-levulinoyl-1-thio- $\beta$-Dxylopyranoside (24). General procedure B with crushed mol sieves $(2.0 \mathrm{~g})$, donor 5 (0.402 g, $0.72 \mathrm{mmol}), \operatorname{AgOTf}(0.378 \mathrm{~g}, 1.47 \mathrm{mmol}), p \mathrm{NO}_{2} \mathrm{PhSCl}(0.141 \mathrm{~g}, 0.74 \mathrm{mmol})$ and acceptor 9 ( $0.286 \mathrm{~g}, 0.65 \mathrm{mmol})$. Reaction time $25 \mathrm{~min}$ then $\mathrm{Et}_{3} \mathrm{~N}(0.30 \mathrm{~mL}, 2.15 \mathrm{mmol})$. Purification by flash column chromatography (pentane/EtOAc 3:2) gave $\mathbf{2 4}$ as a white amorphous solid $(0.547$ g, 95\%). $R_{\mathrm{f}} 0.42$ (hexane/EtOAc 1:1). $[\alpha]^{25} \mathrm{D}-28.1\left(c 0.5, \mathrm{CHCl}_{3}\right) .{ }^{1} \mathrm{H}$ NMR (400 MHz, $\left.\mathrm{CDCl}_{3}\right)$ $\delta 8.02(\mathrm{~d}, J=7.2 \mathrm{~Hz}, 2 \mathrm{H}), 7.94(\mathrm{~d}, J=7.4 \mathrm{~Hz}, 4 \mathrm{H}), 7.59-7.49(\mathrm{~m}, 3 \mathrm{H}), 7.45-7.28(\mathrm{~m}, 11 \mathrm{H})$, $5.69\left(\mathrm{t}, J=6.4 \mathrm{~Hz}, 1 \mathrm{H}, \mathrm{H}^{\mathrm{B}}\right), 5.30\left(\mathrm{td}, J=6.1 \mathrm{~Hz}, J_{5 \mathrm{Beq}, 4 \mathrm{~B}}=4.0 \mathrm{~Hz}, 1 \mathrm{H}, \mathrm{H} 4^{\mathrm{B}}\right), 5.25(\mathrm{~d}, J=8.8$ $\left.\mathrm{Hz}, 1 \mathrm{H}, \mathrm{H} 3^{\mathrm{A}}\right), 5.22-5.19\left(\mathrm{~m}, 1 \mathrm{H}, \mathrm{H} 2^{\mathrm{B}}\right), 4.92-4.87\left(\mathrm{~m}, 2 \mathrm{H}, \mathrm{H}^{\mathrm{A}}, \mathrm{H} 1^{\mathrm{B}}\right), 4.66\left(\mathrm{~d}, J_{\mathrm{A} 1, \mathrm{~A} 2}=9.3 \mathrm{~Hz}\right.$ $\left.1 \mathrm{H}, \mathrm{H} 1^{\mathrm{A}}\right), 4.49\left(\mathrm{dd}, J_{5 \mathrm{Beq}, 5 \mathrm{Bax}}=12.4 \mathrm{~Hz}, J_{5 \mathrm{Beq}, 4 \mathrm{~B}}=3.8 \mathrm{~Hz}, 1 \mathrm{H}, \mathrm{H} 5^{\mathrm{B}}\right.$ eq $), 4.09\left(\mathrm{dd}, J_{5 \text { Aeq }, 5 \mathrm{Aax}}=11.9\right.$ $\mathrm{Hz}, J_{5 \text { Aeq, } 4 \mathrm{~A}}=5.2 \mathrm{~Hz}, 1 \mathrm{H}, \mathrm{H} 5^{\mathrm{A}}$ eq $), 3.90\left(\mathrm{td}, J=9.4 \mathrm{~Hz}, J_{5 \mathrm{Aeq}, 4 \mathrm{~A}}=5.2 \mathrm{~Hz}, 1 \mathrm{H}, \mathrm{H} 4^{\mathrm{A}}\right), 3.75(\mathrm{dd}$, $\left.J_{5 \mathrm{Bax}, 5 \mathrm{Beq}}=12.4 \mathrm{~Hz}, J_{5 \mathrm{Bax}, 4 \mathrm{~B}}=6.0 \mathrm{~Hz}, 1 \mathrm{H}, \mathrm{H}^{\mathrm{B}}{ }_{\mathrm{ax}}\right), 3.32\left(\mathrm{dd}, J_{5 \mathrm{Aax}, 5 \mathrm{Aeq}}=11.7 \mathrm{~Hz}, J_{5 \mathrm{Aeq}, 4 \mathrm{~A}}=9.9 \mathrm{~Hz}\right.$, $\left.1 \mathrm{H}, \mathrm{H}^{\mathrm{A}} \mathrm{ax}\right), 2.88-2.53(\mathrm{~m}, 8 \mathrm{H}), 2.19(\mathrm{~s}, 3 \mathrm{H}), 2.08(\mathrm{~s}, 3 \mathrm{H}) \cdot{ }^{13} \mathrm{C}\left\{{ }^{1} \mathrm{H}\right\} \mathrm{NMR}\left(101 \mathrm{MHz}, \mathrm{CDCl}_{3}\right) \delta$ $206.5,206.4,171.9,171.5,165.7,165.4,165.2,133.6,133.5,133.5,132.9(\times 2), 132.2,130.1$ 
$(\times 2), 130.1(\times 2), 129.9(\times 2), 129.4,129.2,129.1,129.1(\times 2), 128.6(\times 4), 128.3,99.4\left(\mathrm{C} 1^{\mathrm{B}}\right), 86.6$ $\left(\mathrm{C} 1^{\mathrm{A}}\right), 74.9\left(\mathrm{C} 4^{\mathrm{A}}\right), 73.5\left(\mathrm{C}^{\mathrm{A}}\right), 70.3\left(\mathrm{C} 2^{\mathrm{A}}\right), 70.2\left(\mathrm{C} 2^{\mathrm{B}}\right), 69.6\left(\mathrm{C} 3^{\mathrm{B}}\right), 68.7\left(\mathrm{C} 4^{\mathrm{B}}\right), 66.7\left(\mathrm{C}^{\mathrm{A}}\right), 61.1$ $\left(\mathrm{C}^{\mathrm{B}}\right), 37.9,37.8,30.0,29.8,28.1,28.0$. HRMS: $m / z$ calcd for $\mathrm{C}_{47} \mathrm{H}_{46} \mathrm{O}_{15} \mathrm{SNa}[\mathrm{M}+\mathrm{Na}]^{+}$ 905.2449, found 905.2444.

Phenyl 2,3,4-tri-O-benzoyl- $\beta$-D-xylopyranosyl-(1 $\rightarrow 4)$-2,3-di-O-levulinoyl- $\beta$-D-xylopyranosyl$(1 \rightarrow 4)$-2-O-benzoyl-3-O-levulinoyl-1-thio- $\beta$-D-xylopyranoside (25). General procedure B with crushed mol sieves (1.2 g), donor 24 (0.252 g, $0.29 \mathrm{mmol}), \operatorname{AgOTf}(0.148 \mathrm{~g}, 0.58 \mathrm{mmol})$, $p \mathrm{NO}_{2} \mathrm{PhSCl}(0.054 \mathrm{~g}, 0.28 \mathrm{mmol})$ and acceptor 11 (0.114 g, $\left.0.26 \mathrm{mmol}\right)$. Reaction time $25 \mathrm{~min}$ then $\mathrm{Et}_{3} \mathrm{~N}(0.12 \mathrm{~mL}, 0.86 \mathrm{mmol})$. Purification by flash column chromatography (heptane/toluene/EtOAc 2:1:1) gave 25 as a light yellow amorphous solid $(0.162 \mathrm{~g}, 52 \%) . R_{\mathrm{f}}$ 0.35 (hexane/EtOAc 1:1). $[\alpha]^{25} \mathrm{D}-53.8\left(c 1.0, \mathrm{CHCl}_{3}\right) .{ }^{1} \mathrm{H} \mathrm{NMR}\left(400 \mathrm{MHz}, \mathrm{CDCl}_{3}\right) \delta 8.03-7.99$ (m, 6H), 7.96-7.93 (m, 4H), 7.60-7.50 (m, 5H), 7.47-7.32 (m, 12H), 7.28-7.26 (m, 3H), $5.69(\mathrm{t}$, $\left.J=6.6 \mathrm{~Hz}, 1 \mathrm{H}, \mathrm{H}^{\mathrm{C}}\right) 5.30-5.25\left(\mathrm{~m}, 2 \mathrm{H}, \mathrm{H} 3^{\mathrm{A}}, \mathrm{H}^{\mathrm{C}}\right), 5.22\left(\mathrm{dd}, J_{2 \mathrm{C}, 3 \mathrm{C}}=6.5 \mathrm{~Hz}, J_{1 \mathrm{C}, 2 \mathrm{C}}=4.8 \mathrm{~Hz}\right.$, $\left.1 \mathrm{H}, \mathrm{H} 2^{\mathrm{C}}\right), 5.17-5.10\left(\mathrm{~m}, 2 \mathrm{H}, \mathrm{H} 2^{\mathrm{A}}, \mathrm{H} 3^{\mathrm{B}}\right), 4.93\left(\mathrm{~d}, J_{1 \mathrm{~A}, 2 \mathrm{~A}}=8.1 \mathrm{~Hz}, 1 \mathrm{H}, \mathrm{H} 1^{\mathrm{A}}\right), 4.87\left(\mathrm{~d}, J_{1 \mathrm{C}, 2 \mathrm{C}}=4.7\right.$ $\left.\mathrm{Hz}, 1 \mathrm{H}, \mathrm{H} 1^{\mathrm{C}}\right), 4.77\left(\mathrm{dd}, J=8.9,7.0 \mathrm{~Hz}, 1 \mathrm{H}, \mathrm{H} 2^{\mathrm{B}}\right), 4.48-4.44\left(\mathrm{~m}, 2 \mathrm{H}, \mathrm{H} 1^{\mathrm{B}}, \mathrm{H} 5^{\mathrm{C}}\right), 4.22(\mathrm{dd}$, $\left.J_{5 \mathrm{Aeq}, 5 \mathrm{Aax}}=12.0 \mathrm{~Hz}, J_{5 \mathrm{Aeq}, 4 \mathrm{~A}}=4.7 \mathrm{~Hz}, 1 \mathrm{H}, \mathrm{H}^{\mathrm{A}}{ }_{\text {eq }}\right), 3.95\left(\mathrm{dd}, J_{5 \mathrm{Beq}, 5 \mathrm{Bax}}=12.0 \mathrm{~Hz}, J_{5 \mathrm{Beq}, 4 \mathrm{~B}}=5.0\right.$ $\left.\mathrm{Hz}, 1 \mathrm{H}, \mathrm{H}^{\mathrm{B}}{ }_{\mathrm{eq}}\right), 3.86-3.76\left(\mathrm{~m}, 2 \mathrm{H}, \mathrm{H}^{\mathrm{A}}{ }^{\mathrm{A}}, \mathrm{H} 4^{\mathrm{B}}\right), 3.73\left(\mathrm{dd}, J_{5 \mathrm{C}^{\prime}, 5 \mathrm{C}}=12.4 \mathrm{~Hz}, J_{5 \mathrm{C}^{\prime}, 4 \mathrm{C}}=6.2 \mathrm{~Hz}, 1 \mathrm{H}\right.$, $\left.\mathrm{H}^{\circ}{ }^{\mathrm{C}}\right), 3.50\left(\mathrm{dd}, J_{5 \mathrm{Aax}, 5 \mathrm{Aeq}}=12.0 \mathrm{~Hz}, J_{5 \mathrm{ax}, 4 \mathrm{~A}}=8.6 \mathrm{~Hz}, 1 \mathrm{H}, \mathrm{H} 5^{\mathrm{A}}{ }_{\mathrm{ax}}\right), 3.27\left(\mathrm{dd}, J_{5 \mathrm{Bax}, 5 \mathrm{Beq}}=12.0 \mathrm{~Hz}\right.$, $\left.J_{5 \mathrm{Bax}, 4 \mathrm{~B}}=9.1 \mathrm{~Hz}, 1 \mathrm{H}, \mathrm{H}^{\mathrm{B}}{ }_{\mathrm{ax}}\right), 2.75-2.69(\mathrm{~m}, 4 \mathrm{H}), 2.62-2.36(\mathrm{~m}, 8 \mathrm{H}), 2.17(\mathrm{~s}, 3 \mathrm{H}), 2.09(\mathrm{~s}, 3 \mathrm{H})$, 2.04 (s, 3H). ${ }^{13} \mathrm{C}\left\{{ }^{1} \mathrm{H}\right\}$ NMR (101 MHz, $\left.\mathrm{CDCl}_{3}\right) \delta 206.5,206.4,206.1,172.0,171.5,171.3$, $165.7,165.4,165.3,165.2,133.5,133.5,133.5,133.4,132.8,132.7(\times 2), 130.1(\times 2), 130.1(\times 2)$, $130.0(\times 2), 129.9(\times 2), 129.5,129.4,129.2,129.1,129.1(\times 2), 128.6(\times 4), 128.1,100.8\left(\mathrm{C} 1^{\mathrm{B}}\right)$, 
99.3 $\left(\mathrm{C}^{\mathrm{C}}\right), 86.5\left(\mathrm{C}^{\mathrm{A}}\right), 75.0\left(\mathrm{C} 4^{\mathrm{A}}\right), 74.7\left(\mathrm{C} 4^{\mathrm{B}}\right), 72.7\left(\mathrm{C} 3^{\mathrm{A}}\right), 72.2\left(\mathrm{C} 3^{\mathrm{B}}\right), 71.4\left(\mathrm{C} 2^{\mathrm{B}}\right), 70.4\left(\mathrm{C} 2^{\mathrm{A}}\right)$, $70.2\left(\mathrm{C}^{\mathrm{C}}\right), 69.7\left(\mathrm{C}^{\mathrm{C}}\right), 68.8\left(\mathrm{C} 4^{\mathrm{C}}\right), 65.8\left(\mathrm{C}^{\mathrm{A}}\right), 62.9\left(\mathrm{C}^{\mathrm{B}}\right), 61.1\left(\mathrm{C} 5^{\mathrm{C}}\right), 37.9,37.8,37.8,29.9$, 29.8, 29.8, 28.1, 28.0, 27.9. HRMS: $m / z$ calcd for $\mathrm{C}_{64} \mathrm{H}_{64} \mathrm{O}_{22} \mathrm{SNa}[\mathrm{M}+\mathrm{Na}]^{+} 1239.3502$, found 1239.3483.

Phenyl 2,3,4-tri-O-benzoyl- $\beta$-D-xylopyranosyl-(1 $\rightarrow 4)$-2,3-di-O-levulinoyl- $\beta$-D-xylopyranosyl-

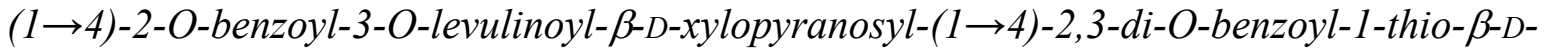
xylopyranoside (26). General procedure B with crushed mol sieves (1.2 g), donor 25 (0.414 g, $0.34 \mathrm{mmol}), \operatorname{AgOTf}(0.175 \mathrm{~g}, 0.68 \mathrm{mmol}), p \mathrm{NO}_{2} \mathrm{PhSCl}(0.064 \mathrm{~g}, 0.34 \mathrm{mmol})$ and acceptor 6 (0.139 g, $0.31 \mathrm{mmol})$. Reaction time $65 \mathrm{~min}$ then $\mathrm{Et}_{3} \mathrm{~N}(0.14 \mathrm{~mL}, 1.02 \mathrm{mmol})$. Purification by flash column chromatography (hexane/toluene/acetone 3:2:1) gave $\mathbf{2 6}$ as a white amorphous solid $(0.358 \mathrm{~g}, 75 \%)$. In addition, donor 25 was reisolated $(0.064 \mathrm{~g}, 15 \%) . R_{\mathrm{f}} 0.32$ (hexane/EtOAc 1:1). $[\alpha]^{25} \mathrm{D}-35.1\left(c\right.$ 1.0, $\left.\mathrm{CHCl}_{3}\right) .{ }^{1} \mathrm{H}$ NMR $\left(400 \mathrm{MHz}, \mathrm{CDCl}_{3}\right) \delta 8.01-7.92(\mathrm{~m}$, 12H), 7.58-7.50 (m, 6H), 7.45-7.31 (m, 14H), 7.28-7.24 (m, 3H), $5.69\left(\mathrm{t}, J=6.6 \mathrm{~Hz}, 1 \mathrm{H}, \mathrm{H}^{\mathrm{D}}\right)$, $5.60\left(\mathrm{t}, J=8.0 \mathrm{~Hz}, 1 \mathrm{H}, \mathrm{H} 3^{\mathrm{A}}\right), 5.32-5.26\left(\mathrm{~m}, 2 \mathrm{H}, \mathrm{H} 2^{\mathrm{A}}, \mathrm{H} 4^{\mathrm{D}}\right), 5.21\left(\mathrm{dd}, J_{2 \mathrm{D}, 3 \mathrm{D}}=6.5 \mathrm{~Hz}, J_{1 \mathrm{D}, 2 \mathrm{D}}=\right.$ $\left.4.8 \mathrm{~Hz}, 1 \mathrm{H}, \mathrm{H} 2^{\mathrm{D}}\right), 5.13-5.07\left(\mathrm{~m}, 2 \mathrm{H}, \mathrm{H}^{\mathrm{B}}, \mathrm{H} 3^{\mathrm{C}}\right), 4.98-4.95\left(\mathrm{~m}, 2 \mathrm{H}, \mathrm{H} 1^{\mathrm{A}}, \mathrm{H} 2^{\mathrm{B}}\right), 4.84\left(\mathrm{~d}, J_{1 \mathrm{D}, 2 \mathrm{D}}=\right.$ $\left.4.7 \mathrm{~Hz}, 1 \mathrm{H}, \mathrm{H}^{\mathrm{D}}\right), 4.73-4.68\left(\mathrm{~m}, 2 \mathrm{H}, \mathrm{H}^{\mathrm{B}}, \mathrm{H} 2^{\mathrm{C}}\right), 4.46\left(\mathrm{dd}, J_{5 \mathrm{Deq}, 5 \mathrm{Dax}}=12.4 \mathrm{~Hz}, J_{5 \mathrm{Deq}, 4 \mathrm{D}}=3.9 \mathrm{~Hz}\right.$, $1 \mathrm{H}, \mathrm{H} 5^{\mathrm{D}}$ eq $), 4.21-4.15\left(\mathrm{~m}, 2 \mathrm{H}, \mathrm{H} 5^{\mathrm{A}}, \mathrm{H} 1^{\mathrm{C}}\right), 4.02-3.96\left(\mathrm{~m}, 1 \mathrm{H}, \mathrm{H} 4^{\mathrm{A}}\right), 3.88\left(\mathrm{dd}, J_{5 \mathrm{C}, 5^{\circ} \mathrm{C}}=11.9 \mathrm{~Hz}\right.$, $\left.J_{5 \mathrm{C}, 4 \mathrm{C}}=5.1 \mathrm{~Hz}, 1 \mathrm{H}, \mathrm{H}^{\mathrm{C}}\right), 3.83-3.77\left(\mathrm{~m}, 1 \mathrm{H}, \mathrm{H}^{\mathrm{C}}\right), 3.72\left(\mathrm{dd}, J_{5 \mathrm{Dax}, 5 \mathrm{Dax}}=12.4 \mathrm{~Hz}, J_{5 \mathrm{Dax}, 4 \mathrm{D}}=6.2\right.$ $\left.\mathrm{Hz}, 1 \mathrm{H}, \mathrm{H}^{\mathrm{D}}{ }_{\mathrm{ax}}\right), 3.56\left(\mathrm{dd}, J_{5 \mathrm{Beq}, 5 \mathrm{Bax}}=11.9 \mathrm{~Hz}, J_{5 \mathrm{Beq}, 4 \mathrm{~B}}=4.6 \mathrm{~Hz}, 1 \mathrm{H}, \mathrm{H} 5^{\mathrm{B}}{ }_{\text {eq }}\right), 3.52-3.44(\mathrm{~m}, 2 \mathrm{H}$, $\left.\mathrm{H}^{\prime}{ }^{\mathrm{A}}, \mathrm{H} 4^{\mathrm{B}}\right), 3.20-3.14\left(\mathrm{~m}, 2 \mathrm{H}, \mathrm{H} 5^{\circ}{ }^{\mathrm{C}}, \mathrm{H}^{\mathrm{B}}{ }_{\mathrm{ax}}\right), 2.73-2.69(\mathrm{~m}, 4 \mathrm{H}), 2.60-2.31(\mathrm{~m}, 8 \mathrm{H}), 2.17(\mathrm{~s}$, 3H), 2.08 (s, 3H), $2.03(\mathrm{~s}, 3 \mathrm{H}) .{ }^{13} \mathrm{C}\left\{{ }^{1} \mathrm{H}\right\}$ NMR (101 MHz, $\left.\mathrm{CDCl}_{3}\right) \delta 206.4,206.3,206.1,172.0$, $171.6,171.3,165.6,165.4,165.4,165.3,165.1,165.1,133.6,133.5,133.5,133.4,133.4,133.3$, 
132.8, $132.6(\times 2), 130.1(\times 2), 130.0(\times 2), 130.0(\times 2), 129.9(\times 2), 129.9(\times 2), 129.9(\times 2), 129.6$, $129.5,129.4,129.4,129.2,129.1,129.1(\times 2), 128.6(\times 2), 128.6(\times 4), 128.5(\times 2), 128.1,100.5$ $\left(\mathrm{C} 1^{\mathrm{C}}, J_{\mathrm{C}-\mathrm{H}}=161 \mathrm{~Hz}\right), 100.3\left(\mathrm{C}^{\mathrm{B}}, J_{\mathrm{C}-\mathrm{H}}=164 \mathrm{~Hz}\right), 99.3\left(\mathrm{C} 1^{\mathrm{D}}, J_{\mathrm{C}-\mathrm{H}}=165 \mathrm{~Hz}\right), 86.7\left(\mathrm{C} 1^{\mathrm{A}}, J_{\mathrm{C}-\mathrm{H}}=\right.$ $159 \mathrm{~Hz}), 75.0\left(\mathrm{C}^{\mathrm{A}}\right), 74.7\left(\mathrm{C}^{\mathrm{C}}\right), 74.5\left(\mathrm{C}^{\mathrm{B}}\right), 73.0\left(\mathrm{C} 3^{\mathrm{A}}\right), 72.2\left(\mathrm{C} 3^{\mathrm{C}}\right), 71.4\left(\mathrm{C}^{\mathrm{B}}\right), 71.2\left(\mathrm{C} 2^{\mathrm{C}}\right)$, $71.0\left(\mathrm{C} 2^{\mathrm{B}}\right), 70.4,70.2\left(\mathrm{C} 2^{\mathrm{D}}, \mathrm{C} 4^{\mathrm{D}}\right), 69.7\left(\mathrm{C}^{\mathrm{D}}\right), 68.8\left(\mathrm{C} 2^{\mathrm{A}}\right), 65.8\left(\mathrm{C} 5^{\mathrm{A}}\right), 62.9\left(\mathrm{C} 5^{\mathrm{C}}\right), 62.0\left(\mathrm{C} 5^{\mathrm{B}}\right)$, $61.1\left(\mathrm{C}^{\mathrm{D}}\right), 37.8,37.8(\times 2), 29.9,29.8,29.8,28.0,27.9,27.8 . \mathrm{HRMS}: \mathrm{m} / z$ calcd for $\mathrm{C}_{83} \mathrm{H}_{80} \mathrm{O}_{28} \mathrm{SNa}[\mathrm{M}+\mathrm{Na}]^{+} 1579.4449$, found 1579.4425 .

Phenyl 2,3,4-tri-O-benzoyl- $\beta$-D-xylopyranosyl-( $1 \rightarrow 4)$ - $\beta$-D-xylopyranosyl-( $1 \rightarrow 4)$-2-O-benzoyl- $\beta$ D-xylopyranosyl-(1 $\rightarrow 4)$-2,3-di-O-benzoyl-1-thio- $\beta$-D-xylopyranoside (27). General procedure C with tetrasaccharide $26(0.252 \mathrm{~g}, 0.16 \mathrm{mmol})$ and $50 \%$ solution of hydrazine hydrate $(0.10 \mathrm{~mL}$, $1.59 \mathrm{mmol}$ ). Reaction time $10 \mathrm{~min}$. Eluent for TLC (hexane/toluene/acetone 2:1:1). The product was a white powder $(0.202 \mathrm{~g}, 99 \%) . R_{\mathrm{f}} 0.64$ (hexane/acetone $\left.1: 1\right) .[\alpha]^{25} \mathrm{D}-9.7\left(c 1.0, \mathrm{CHCl}_{3}\right)$. ${ }^{1} \mathrm{H}$ NMR (400 MHz, $\left.\mathrm{CDCl}_{3}\right) \delta 8.03-7.91(\mathrm{~m}, 12 \mathrm{H}), 7.59-7.33(\mathrm{~m}, 20 \mathrm{H}), 7.28-7.25(\mathrm{~m}, 3 \mathrm{H})$, $5.80\left(\mathrm{t}, J=8.5 \mathrm{~Hz}, 1 \mathrm{H}, \mathrm{H} 3^{\mathrm{D}}\right), 5.58\left(\mathrm{t}, J_{2 \mathrm{~A}, 3 \mathrm{~A}}=7.7 \mathrm{~Hz}, 1 \mathrm{H}, \mathrm{H} 3^{\mathrm{A}}\right), 5.40-5.34\left(\mathrm{~m}, 2 \mathrm{H}, \mathrm{H} 2^{\mathrm{D}}, \mathrm{H}^{\mathrm{D}}\right)$, $5.31\left(\mathrm{t}, J=7.8 \mathrm{~Hz}, 1 \mathrm{H}, \mathrm{H} 2^{\mathrm{A}}\right), 4.99\left(\mathrm{~d}, J_{1 \mathrm{~A}, 2 \mathrm{~A}}=7.8 \mathrm{~Hz}, 1 \mathrm{H}, \mathrm{H} 1^{\mathrm{A}}\right), 4.92\left(\mathrm{dd}, J_{2 \mathrm{~B}, 3 \mathrm{~B}}=8.6 \mathrm{~Hz}, J_{1 \mathrm{~B}, 2 \mathrm{~B}}\right.$ $\left.=7.0 \mathrm{~Hz}, 1 \mathrm{H}, \mathrm{H} 2^{\mathrm{B}}\right), 4.79\left(\mathrm{~d}, J_{1 \mathrm{D}, 2 \mathrm{D}}=6.6 \mathrm{~Hz}, 1 \mathrm{H}, \mathrm{H}^{\mathrm{D}}\right), 4.63\left(\mathrm{~d}, J_{1 \mathrm{~B}, 2 \mathrm{~B}}=6.9 \mathrm{~Hz}, 1 \mathrm{H}, \mathrm{H} 1^{\mathrm{B}}\right), 4.50$ $\left(\mathrm{dd}, J_{5 \mathrm{Deq}, 5 \mathrm{Dax}}=11.9 \mathrm{~Hz}, J_{5 \mathrm{Deq}, 4 \mathrm{D}}=4.9 \mathrm{~Hz}, 1 \mathrm{H}, \mathrm{H}^{\mathrm{D}}\right.$ eq $), 4.18\left(\mathrm{dd}, J_{5 \mathrm{Aeq}, 5 \mathrm{Aax}}=12.1 \mathrm{~Hz}, J_{5 \mathrm{Aeq}, 4 \mathrm{~A}}=\right.$ 4.6 Hz, 1H, H5 ${ }^{\mathrm{A}}$ ) $), 4.14\left(\mathrm{~d}, J_{1 \mathrm{C}, 2 \mathrm{C}}=7.4 \mathrm{~Hz}, 1 \mathrm{H}, \mathrm{H} 1^{\mathrm{C}}\right), 4.01-3.95\left(\mathrm{~m}, 1 \mathrm{H}, \mathrm{H} 4^{\mathrm{A}}\right), 3.75-3.42(\mathrm{~m}$, $\left.9 \mathrm{H}, \mathrm{H} 5^{\mathrm{A}}{ }_{\mathrm{ax}}, \mathrm{H} 3^{\mathrm{B}}, \mathrm{H} 4^{\mathrm{B}}, \mathrm{H} 5^{\mathrm{B}}, \mathrm{H} 3^{\mathrm{C}}, \mathrm{H} 4^{\mathrm{C}}, \mathrm{H} 5^{\mathrm{C}}, \mathrm{H} 5^{\mathrm{D}}{ }_{\mathrm{ax}}\right), 3.37-3.33\left(\mathrm{~m}, 1 \mathrm{H}, \mathrm{H} 2^{\mathrm{C}}\right), 3.15-3.09(\mathrm{~m}, 2 \mathrm{H}$, $\left.\mathrm{H} 5{ }^{\mathrm{B}}, \mathrm{H} 5{ }^{, \mathrm{C}}\right) .{ }^{13} \mathrm{C}\left\{{ }^{1} \mathrm{H}\right\} \mathrm{NMR}\left(101 \mathrm{MHz}, \mathrm{CDCl}_{3}\right) \delta 165.7,165.6,165.6,165.5,165.3,165.2$, $133.7,133.6,133.5,133.4,133.3,133.3,132.9,132.5(\times 2), 130.0(\times 2), 130.0(\times 2), 129.9(\times 2)$, $129.9(\times 3), 129.8(\times 2), 129.6,129.6,129.4,129.1(\times 2), 129.0,129.0,128.9,128.7(\times 2), 128.6$ 
$(\times 3), 128.5(\times 2), 128.5(\times 3), 128.1,102.5\left(\mathrm{C1}^{\mathrm{C}}\right), 101.4\left(\mathrm{C} 1^{\mathrm{B}}\right), 101.0\left(\mathrm{C} 1^{\mathrm{D}}\right), 86.6\left(\mathrm{C1}^{\mathrm{A}}\right), 78.8$

$\left(\mathrm{C} 4^{\mathrm{C}}\right), 77.4\left(\mathrm{C}^{\mathrm{B}}\right), 75.7\left(\mathrm{C} 4^{\mathrm{A}}\right), 74.0\left(\mathrm{C} 3^{\mathrm{C}}\right), 73.7\left(\mathrm{C} 2^{\mathrm{B}}\right), 72.8,72.7\left(\mathrm{C}^{\mathrm{A}}, \mathrm{C} 3^{\mathrm{B}}\right), 72.1\left(\mathrm{C} 2^{\mathrm{C}}\right), 71.1$, $70.9\left(\mathrm{C}^{\mathrm{D}}, \mathrm{C}^{\mathrm{D}}\right), 70.3\left(\mathrm{C} 2^{\mathrm{A}}\right), 69.3\left(\mathrm{C} 4^{\mathrm{D}}\right), 65.7\left(\mathrm{C}^{\mathrm{A}}\right), 63.1\left(\mathrm{C}^{\mathrm{B}} / \mathrm{C} 5^{\mathrm{C}}\right), 63.0\left(\mathrm{C} 5^{\mathrm{B}} / \mathrm{C} 5^{\mathrm{C}}\right), 62.5$ (C5 ${ }^{\mathrm{D}}$ ). HRMS: $\mathrm{m} / z$ calcd for $\mathrm{C}_{68} \mathrm{H}_{62} \mathrm{O}_{22} \mathrm{SNa}[\mathrm{M}+\mathrm{Na}]^{+} 1285.3345$, found 1285.3335 .

Phenyl 2,3,4-tri-O-benzoyl- $\beta$-D-xylopyranosyl-( $(1 \rightarrow 4)$-[2,3,5-tri-O-benzoyl- $\alpha$-Larabinofuranosyl-(1-2)]-[2,3,5-tri-O-benzoyl- $\alpha$-L-arabinofuranosyl-( $(1 \rightarrow 3)]-\beta$-D-

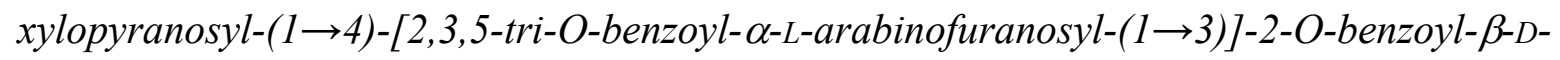
xylopyranosyl-(1 $\rightarrow 4)$-2,3-di-O-benzoyl-1-thio- $\beta$-D-xylopyranoside (28). General procedure D with acceptor 27 (0.088 g, $0.069 \mathrm{mmol})$, donor $4(0.175 \mathrm{~g}, 0.28 \mathrm{mmol})$ and TMSOTf in dry $\mathrm{CH}_{2} \mathrm{Cl}_{2}(0.17 \mathrm{~mL}, c=0.04 \mathrm{mmol} / \mathrm{mL})$. Reaction time $105 \mathrm{~min}$ then $\mathrm{Et}_{3} \mathrm{~N}(0.02 \mathrm{~mL}, 0.14 \mathrm{mmol})$. Purification by flash column chromatography (hexane/toluene/acetone 3:3:1) gave $\mathbf{2 8}$ as a white amorphous solid (0.168 g, 91\%). $R_{\mathrm{f}} 0.26$ (hexane/toluene/acetone 3:3:1). $[\alpha]^{25} \mathrm{D}-58.4(c 1.0$, $\left.\mathrm{CHCl}_{3}\right) .{ }^{1} \mathrm{H}$ NMR $\left(400 \mathrm{MHz}, \mathrm{CDCl}_{3}\right) \delta 8.22(\mathrm{~d}, J=8.1 \mathrm{~Hz}, 2 \mathrm{H}), 8.12-8.07(\mathrm{~m}, 6 \mathrm{H}), 8.03-7.93$ (m, 14H), 7.86-7.79 (m, 8H), $7.73(\mathrm{~d}, J=7.3 \mathrm{~Hz}, 2 \mathrm{H}), 7.58(\mathrm{~d}, J=7.4 \mathrm{~Hz}, 1 \mathrm{H}), 7.62-7.10(\mathrm{~m}$, 46H), $7.03(\mathrm{t}, J=7.8 \mathrm{~Hz}, 1 \mathrm{H}), 5.69\left(\mathrm{~s}, 1 \mathrm{H}, \mathrm{H} 1^{\mathrm{F}}\right), 5.63\left(\mathrm{t}, J=9.6 \mathrm{~Hz}, 1 \mathrm{H}, \mathrm{H} 4^{\mathrm{D}}\right), 5.58(\mathrm{~s}, 1 \mathrm{H}$, $\left.\mathrm{H}^{\mathrm{G}}{ }^{\mathrm{G}}\right), 5.53(\mathrm{~s}, 1 \mathrm{H}), 5.51(\mathrm{~d}, J=4.4 \mathrm{~Hz}, 1 \mathrm{H}), 5.40-5.39\left(\mathrm{~m}, 3 \mathrm{H}, \mathrm{H}^{\mathrm{A}}\right), 5.37-5.34(\mathrm{~m}, 2 \mathrm{H})$, 5.21-5.16 (m, 4H, H2 $\left.2^{\mathrm{A}}, \mathrm{H} 2^{\mathrm{D}}, \mathrm{H} 1^{\mathrm{E}}\right), 5.13-5.03\left(\mathrm{~m}, 3 \mathrm{H}, \mathrm{H} 2^{\mathrm{B}}, \mathrm{H} 4^{\mathrm{D}}\right), 5.00-4.93(\mathrm{~m}, 2 \mathrm{H}), 4.86-4.74$ $\left(\mathrm{m}, 7 \mathrm{H}, \mathrm{H} 1^{\mathrm{A}}\right), 4.31\left(\mathrm{~d}, J_{\mathrm{B} 1, \mathrm{~B} 2}=6.6 \mathrm{~Hz}, 1 \mathrm{H}, \mathrm{H} 1^{\mathrm{B}}\right), 4.02-3.96\left(\mathrm{~m}, 2 \mathrm{H}, \mathrm{H} 5^{\mathrm{A}}, \mathrm{H} 1^{\mathrm{D}}\right), 3.90(\mathrm{t}, J=8.7$ $\left.\mathrm{Hz}, 1 \mathrm{H}, \mathrm{H} 3^{\mathrm{B}}\right), 3.83-3.78\left(\mathrm{~m}, 2 \mathrm{H}, \mathrm{H}^{\mathrm{C}}, \mathrm{H}^{\mathrm{C}}\right), 3.58-3.52\left(\mathrm{~m}, 2 \mathrm{H}, \mathrm{H} 4^{\mathrm{B}}, \mathrm{H} 2^{\mathrm{C}}\right), 3.45(\mathrm{td}, J=9.5,5.0$ $\left.\mathrm{Hz}, 1 \mathrm{H}, \mathrm{H} 4^{\mathrm{C}}\right), 3.41-3.35\left(\mathrm{~m}, 2 \mathrm{H}, \mathrm{H}^{\mathrm{A}}{ }^{\mathrm{A}}, \mathrm{H} 5^{\mathrm{D}}\right), 3.32-3.24\left(\mathrm{~m}, 2 \mathrm{H}, \mathrm{H} 5^{\circ}{ }^{\mathrm{A}}, \mathrm{H} 5^{\mathrm{B}}{ }_{\text {eq }}\right), 3.09-3.00$ (m, $\left.2 \mathrm{H}, \mathrm{H}^{\mathrm{C}}, \mathrm{H} 5^{\circ} \mathrm{D}\right), 2.92\left(\mathrm{dd}, J_{5 \mathrm{Bax}, 5 \mathrm{Beq}}=12.2 \mathrm{~Hz}, J_{5 \mathrm{Bax}, 4 \mathrm{~B}}=7.5 \mathrm{~Hz}, 1 \mathrm{H}, \mathrm{H}^{\mathrm{B}}{ }_{\mathrm{ax}}\right), 2.45(\mathrm{t}, J=10.8 \mathrm{~Hz}$, $\left.1 \mathrm{H}, \mathrm{H} 5^{\circ} \mathrm{C}\right) .{ }^{13} \mathrm{C}\left\{{ }^{1} \mathrm{H}\right\} \mathrm{NMR}\left(101 \mathrm{MHz}, \mathrm{CDCl}_{3}\right) \delta 166.6(\times 2), 166.4,166.3,165.9,165.8,165.8$, 
165.6, 165.5, 165.3, 165.2, 164.9, 164.9, 164.9, 164.4, 134.1, 134.0, 133.5, 133.4, 133.4, 133.3, 133.3, 133.1, 133.0, 132.9, 132.8, 132.1, 130.3, 130.2, 130.1, 130.1, 130.0, 130.0, 130.0, 129.9, $129.9,129.9,129.8,129.8,129.7,129.7,129.6,129.5,129.3,129.3,129.2,129.1,129.0,129.0$, $129.0,128.9,128.6,128.6,128.5,128.5,128.4,128.3,128.3,128.2,128.2,106.1\left(\mathrm{C}^{\mathrm{G}}\right), 105.7$ $\left(\mathrm{C} 1^{\mathrm{F}}\right), 105.4\left(\mathrm{C1}^{\mathrm{E}}\right), 100.1\left(\mathrm{C} 1^{\mathrm{B}}\right), 99.8\left(\mathrm{C1}^{\mathrm{D}}\right), 99.6\left(\mathrm{C}^{\mathrm{C}}\right), 86.2\left(\mathrm{C}^{\mathrm{A}}\right)$, 82.0, 81.9, $81.9(\times 2), 81.7$, 81.6, 78.9, 78.7, 78.4, $77.4\left(\mathrm{C}^{\mathrm{C}}\right), 76\left(\mathrm{C}^{\mathrm{C}}\right), 75.5\left(\mathrm{C} 3^{\mathrm{B}}\right), 74.7(\mathrm{C} 4 \mathrm{C}), 73.6,73.3,73.1(\times 2),\left(\mathrm{C} 3^{\mathrm{A}}\right.$, $\left.\mathrm{C}^{\mathrm{A}}, \mathrm{C}^{\mathrm{B}}, \mathrm{C} 4^{\mathrm{B}}\right), 72.4\left(\mathrm{C} 3^{\mathrm{D}}\right), 71.1,70.3\left(\mathrm{C} 2^{\mathrm{A}}, \mathrm{C}^{\mathrm{D}}\right), 69.8\left(\mathrm{C} 4^{\mathrm{D}}\right), 66.1\left(\mathrm{C} 5^{\mathrm{A}}\right), 64.4,64.1,64.1$ $\left(\mathrm{C}^{\mathrm{E}}, \mathrm{C}^{\mathrm{F}}, \mathrm{C}^{\mathrm{G}}\right), 63.1,62.8\left(\mathrm{C}^{\mathrm{C}}, \mathrm{C}^{\mathrm{D}}\right), 62.0\left(\mathrm{C} 5^{\mathrm{B}}\right)$. HRMS: $m / z$ calcd for $\mathrm{C}_{146} \mathrm{H}_{122} \mathrm{O}_{43} \mathrm{SNa}[\mathrm{M}+$ $\mathrm{Na}]^{+}$2617.6973, found 2617.6916.

2,3,4-Tri-O-benzoyl- $\beta$-D-xylopyranosyl-( $(\rightarrow 4)$-[2,3,5-tri-O-benzoyl- $\alpha$-L-arabinofuranosyl$(1 \rightarrow 3)]$-2-O-benzoyl- $\beta$-D-xylopyranosyl-( $(1 \rightarrow 4)$-[2,3,5-tri-O-benzoyl- $\alpha$-L-arabinofuranosyl$(1 \rightarrow 3)]$-2-O-benzoyl- $\beta$-D-xylopyranosyl-( $1 \rightarrow 4)$-[2,3,5-tri-O-benzoyl- $\alpha$-L-arabinofuranosyl$(1 \rightarrow 3)$ ]-2-O-benzoyl-D-xylopyranose (29). General procedure E with thioglycoside 19 (0.081 g, $0.031 \mathrm{mmol})$, NBS (0.030 g, $0.17 \mathrm{mmol})$, additional NBS added after $2 \mathrm{~h}$ and $15 \mathrm{~min}(0.026 \mathrm{~g}$, $0.15 \mathrm{mmol}$ ). Reaction time $3 \mathrm{~h}$. Purification by flash column chromatography (heptane/acetone 3:2) gave 29 as a white amorphous solid $(0.072 \mathrm{~g}, \alpha / \beta$ ratio $\sim 1: 0.3,92 \%) . R_{\mathrm{f}} 0.42$ (heptane/EtOAc 1:1). ${ }^{1} \mathrm{H}$ NMR (400 MHz, $\left.\mathrm{CDCl}_{3}\right) \delta 8.18-7.80$ (m, 39H), 7.64-7.19 (m, 58.5H), $5.78(\mathrm{t}, J=8.9 \mathrm{~Hz}, 0.2 \mathrm{H}) 5.71\left(\mathrm{t}, J=9.3 \mathrm{~Hz}, 1 \mathrm{H}, \mathrm{H} 3^{\mathrm{D}}\right), 5.53-5.49\left(\mathrm{~m}, 4 \mathrm{H}, \mathrm{H} 1^{\mathrm{E}} / \mathrm{H} 1^{\mathrm{F}}\right), 5.46-5.42$ (m, 2H), 5.39-5.35 (m, 4H, H1 $\left.{ }^{\mathrm{A}}\right), 5.30-5.28\left(\mathrm{~m}, 2 \mathrm{H}, \mathrm{H} 2^{\mathrm{D}}, \mathrm{H} 1^{\mathrm{G}}\right), 5.26\left(\mathrm{~s}, 1 \mathrm{H}, \mathrm{H} 1^{\mathrm{E}} / \mathrm{H} 1^{\mathrm{F}}\right), 5.16$ (td, $\left.J=9.3,5.5 \mathrm{~Hz}, 1 \mathrm{H}, \mathrm{H} 4^{\mathrm{D}}\right), 5.09-4.84\left(\mathrm{~m}, 15 \mathrm{H}, \mathrm{H} 2^{\mathrm{A}}, \mathrm{H} 2^{\mathrm{B}}, \mathrm{H} 2^{\mathrm{C}}\right), 4.48(\mathrm{~d}, J=7.8 \mathrm{~Hz}, 0.3 \mathrm{H}$, $\left.\mathrm{H}^{\mathrm{A}}{ }_{\beta}\right), 4.36\left(\mathrm{t}, J=9.3 \mathrm{~Hz}, 1 \mathrm{H}, \mathrm{H} 3^{\mathrm{A}}\right), 4.25\left(\mathrm{dd}, J=8.0,3.7 \mathrm{~Hz}, 1 \mathrm{H}, \mathrm{H} 1^{\mathrm{B}}\right), 4.11-4.05(\mathrm{~m}, 2 \mathrm{H}$, $\left.\mathrm{H} 1^{\mathrm{D}}\right), 3.91-3.81\left(\mathrm{~m}, 4 \mathrm{H}, \mathrm{H} 3^{\mathrm{B}}, \mathrm{H}^{\mathrm{C}}, \mathrm{H} 5^{\mathrm{D}}\right), 3.76\left(\mathrm{td}, J=9.5,5.0 \mathrm{~Hz}, 1 \mathrm{H}, \mathrm{H} 4^{\mathrm{A}}\right), 3.58-3.46(\mathrm{~m}, 4 \mathrm{H}$, 
$\left.\mathrm{H}^{\mathrm{A}}, \mathrm{H} 5^{\mathrm{A}}{ }^{\mathrm{A}}, \mathrm{H} 1^{\mathrm{C}}, \mathrm{H} 4^{\mathrm{C}}\right), 3.37-3.29$ (m, 2H, H4$\left.{ }^{\mathrm{B}}, \mathrm{H} 5^{\mathrm{C}}\right), 3.24-3.19\left(\mathrm{~m}, 1 \mathrm{H}, \mathrm{H} 5^{\mathrm{D}}\right), 3.12(\mathrm{dd}, J=$ 11.9, 5.6 Hz, 1H, H5 $\left.{ }^{\mathrm{B}}\right), 2.99-2.94(\mathrm{~m}, 0.3 \mathrm{H}), 2.66-2.59\left(\mathrm{~m}, 2 \mathrm{H}, \mathrm{H} 5^{\circ}{ }^{\mathrm{B}}, \mathrm{H} 5^{\circ}{ }^{\mathrm{C}}\right) .{ }^{13} \mathrm{C}\left\{{ }^{1} \mathrm{H}\right\} \mathrm{NMR}$ $\left(101 \mathrm{MHz} \mathrm{CDCl}_{3}\right) \delta 167.0,166.4,166.4,166.4,166.1,165.9,165.8,165.8,165.8,165.6,165.2$, $165.1,165.1,165.0,164.8,164.5,164.4,164.2,133.9,133.9,133.7,133.7,133.5,133.4,133.4$, $133.3,133.2,133.2,133.2,133.0,133.0,132.9,130.4,130.4,130.2,130.2,130.1,130.1,130.0$ $129.9,129.9,129.8,129.8,129.8,129.7,129.6,129.5,129.5,129.5,129.3,129.2,129.2,129.1$, $129.1,129.0,129.0,129.0,128.9,128.9,128.9,128.7,128.6,128.5,128.5,128.4,128.4,128.4$, $128.3,128.3,128.3,128.3,106.1\left(\mathrm{C}^{\mathrm{G}}\right), 105.7,105.6\left(\mathrm{C} 1^{\mathrm{E}}, \mathrm{C} 1^{\mathrm{F}}\right), 100.4\left(\mathrm{C}_{\beta}\right), 100.3\left(\mathrm{C} 1^{\mathrm{B}}\right), 100.0$ $\left(\mathrm{C} 1^{\mathrm{C}}\right), 100.0\left(\mathrm{C}_{\beta}\right), 99.9\left(\mathrm{C1}^{\mathrm{D}}\right), 96.4\left(\mathrm{C}_{\beta}\right), 90.5\left(\mathrm{C} 1^{\mathrm{A}}\right), 82.8,82.7,82.7,81.8\left(\mathrm{C}_{\beta}\right), 81.7,81.5$, 80.8, 78.3, 78.3, 78.2, 78.1 $\left(\mathrm{C}_{\beta}\right), 76.3\left(\mathrm{C}_{\beta}\right), 75.4\left(\mathrm{C}_{\beta}\right), 75.3,73.3\left(\mathrm{C}^{\mathrm{B}}, \mathrm{C}^{\mathrm{C}}\right), 74.7\left(\mathrm{C}_{\beta}\right), 74.5$, 74.3, $74.3\left(\mathrm{C}^{\mathrm{A}}, \mathrm{C}^{\mathrm{B}}, \mathrm{C}^{\mathrm{C}}\right), 74.2\left(\mathrm{C}_{\beta}\right), 74.2\left(\mathrm{C}_{\beta}\right), 74.1\left(\mathrm{C}_{\beta}\right), 73.4\left(\mathrm{C} 2^{\mathrm{B}}\right), 73.0\left(\mathrm{C} 2^{\mathrm{C}}\right), 72.1,72.0$ $\left(\mathrm{C} 2^{\mathrm{A}}, \mathrm{C}^{\mathrm{D}}\right), 71.2\left(\mathrm{C}^{\mathrm{D}}\right), 69.8\left(\mathrm{C} 4^{\mathrm{D}}\right), 64.0,63.9,63.9,63.8\left(\mathrm{C} 5^{\mathrm{E}}, \mathrm{C} 5^{\mathrm{F}}, \mathrm{C}^{\mathrm{G}}\right), 63.3\left(\mathrm{C}_{\beta}\right), 63.1,63.0$, $63.0\left(\mathrm{C}^{\mathrm{B}}, \mathrm{C}^{\mathrm{C}}, \mathrm{C}^{\mathrm{D}}\right), 59.3\left(\mathrm{C}^{\mathrm{A}}\right) . \mathrm{HRMS}: \mathrm{m} / z$ calcd for $\mathrm{C}_{140} \mathrm{H}_{118} \mathrm{O}_{44} \mathrm{SNa}[\mathrm{M}+\mathrm{Na}]^{+} 2525.6888$ found 2525.6876 .

\section{2,3,4-Tri-O-benzoyl- $\beta$-D-xylopyranosyl-(1 $\rightarrow 4)$-[2,3,5-tri-O-benzoyl- $\alpha$-L-arabinofuranosyl- $(1 \rightarrow 3)]-2-O$-benzoyl- $\beta$-D-xylopyranosyl-(1 $\rightarrow 4)$-[2,3,5-tri-O-benzoyl- $\alpha$-L-arabinofuranosyl-

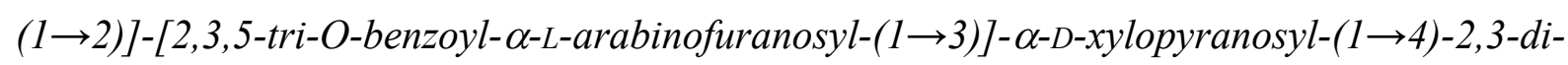
O-benzoyl-D-xylopyranose (30). General procedure E with thioglycoside $23(0.200 \mathrm{~g}, 0.077$ mmol), acetone/ $\mathrm{H}_{2} \mathrm{O} 9: 1(1.23 \mathrm{~mL})$, NBS (0.055 g, $\left.0.31 \mathrm{mmol}\right)$, additional NBS added after 30 $\min (0.030 \mathrm{~g}, 0.17 \mathrm{mmol})$. Reaction time $60 \mathrm{~min}$. Eluent for TLC and flash column chromatography (heptane/acetone 3:2). Product was isolated as a white amorphous solid (0.181 $\mathrm{g}, \alpha / \beta$ ratio $\sim 1: 0.35,93 \%) . R_{\mathrm{f}} 0.45$ (heptane/EtOAc $\left.1: 1\right) .{ }^{1} \mathrm{H}$ NMR $\left(400 \mathrm{MHz}, \mathrm{CDCl}_{3}\right) \delta$ 
8.19-8.18 (m, 2H), 8.10-7.80 (m, 27H), 7.78-7.74 (m, 1H), 7.69-7.64 (m, 2H), 7.59-7.20 (m, 39H), 7.18-7.07 (m, 4H), 5.86-5.82 (m, 1H, H3 $\left.{ }^{\mathrm{A}}\right), 5.79-5.78\left(\mathrm{~m}, 1 \mathrm{H}, \mathrm{H} 1^{\mathrm{F}}\right), 5.69(\mathrm{t}, J=9.2 \mathrm{~Hz}$, $\left.1 \mathrm{H}, \mathrm{H} 3^{\mathrm{D}}\right), 5.63-5.61\left(\mathrm{~m}, 1 \mathrm{H}, \mathrm{H} 1^{\mathrm{E}}\right), 5.55-5.50(\mathrm{~m}, 6 \mathrm{H}), 5.36-5.35\left(\mathrm{~m}, 2 \mathrm{H}, \mathrm{H} 1^{\mathrm{A}}\right), 5.26(\mathrm{~s}, 1 \mathrm{H}$, $\left.\mathrm{H}^{\mathrm{G}}\right), 5.23\left(\mathrm{dd}, J_{2 \mathrm{D}, 3 \mathrm{D}}=9.3 \mathrm{~Hz}, J_{1 \mathrm{D}, 2 \mathrm{D}}=7.4 \mathrm{~Hz}, 1 \mathrm{H}, \mathrm{H} 2^{\mathrm{D}}\right), 5.17\left(\mathrm{td}, J_{3 \mathrm{D}, 4 \mathrm{D}}=9.2 \mathrm{~Hz}, J_{4 \mathrm{D}, 5 \mathrm{D}}=5.5\right.$ $\left.\mathrm{Hz}, 1 \mathrm{H}, \mathrm{H}^{\mathrm{D}}\right), 5.12-5.07(\mathrm{~m}, 2 \mathrm{H}), 5.05-4.81\left(\mathrm{~m}, 7 \mathrm{H}, \mathrm{H} 2^{\mathrm{A}}, \mathrm{H} 2^{\mathrm{C}}\right), 4.76-4.64(\mathrm{~m}, 2 \mathrm{H}), 4.57(\mathrm{~d}, J=$ $\left.7.3 \mathrm{~Hz}, 0.33 \mathrm{H}, \mathrm{H} 1_{\beta}^{\mathrm{A}}\right), 4.31\left(\mathrm{t}, J_{1 \mathrm{~B}, 2 \mathrm{~B}}=7.5 \mathrm{~Hz}, 1 \mathrm{H}, \mathrm{H} 1^{\mathrm{B}}\right), 4.14\left(\mathrm{~d}, J_{1 \mathrm{D}, 2 \mathrm{D}}=7.3 \mathrm{~Hz}, 1 \mathrm{H}, \mathrm{H} 1^{\mathrm{D}}\right), 4.00$ $(\mathrm{dd}, J=11.9,5.3 \mathrm{~Hz}, 0.40 \mathrm{H}), 3.93-3.82\left(\mathrm{~m}, 6 \mathrm{H}, \mathrm{H}^{\mathrm{A}}, \mathrm{H} 5^{\mathrm{A}}, \mathrm{H} 3^{\mathrm{B}}, \mathrm{H} 1^{\mathrm{C}}, \mathrm{H}^{\mathrm{C}}, \mathrm{H} 5^{\mathrm{D}}\right), 3.74-3.69(\mathrm{~m}$, $\left.1 \mathrm{H}, \mathrm{H} 5^{\circ} \mathrm{A}\right), 3.66\left(\mathrm{dd}, J_{2 \mathrm{~B}, 3 \mathrm{~B}}=9.6 \mathrm{~Hz}, J_{1 \mathrm{~B}, 2 \mathrm{~B}}=6.9 \mathrm{~Hz}, 1 \mathrm{H}, \mathrm{H} 2^{\mathrm{B}}\right), 3.54-3.38\left(\mathrm{~m}, 3 \mathrm{H}, \mathrm{H} 4^{\mathrm{A}}, \mathrm{H} 4^{\mathrm{C}}\right)$, 3.36-3.24 (m, 2H, H5 $\left.{ }^{\mathrm{B}}, \mathrm{H} 5^{\mathrm{D}} \mathrm{D}\right), 3.07-3.00\left(\mathrm{~m}, 1 \mathrm{H}, \mathrm{H} 5^{\mathrm{C}}\right), 2.74-2.63\left(\mathrm{~m}, 1 \mathrm{H}, \mathrm{H} 5^{\mathrm{B}}\right), 2.53(\mathrm{t}, J=$ $\left.11.1 \mathrm{~Hz}, 1 \mathrm{H}, \mathrm{H} 5{ }^{\circ} \mathrm{C}\right) .{ }^{13} \mathrm{C}\left\{{ }^{1} \mathrm{H}\right\} \mathrm{NMR}\left(101 \mathrm{MHz}, \mathrm{CDCl}_{3}\right) \delta 166.9,166.7,166.5,166.4,166.1$, $165.9,165.9,165.8,165.8,165.7,165.7,165.6,165.6,165.4,165.4,165.4,165.1,165.0,164.6$ $164.2,133.7,133.6,133.6,133.5,133.4,133.4,133.3,133.2,133.1,133.0,132.9,132.8,130.3$, $130.2,130.2,130.1,130.0,130.0,129.9,129.9,129.9,129.8,129.8,129.8,129.6,129.6,129.3$, $129.2,129.2,129.2,129.1,129.1,129.1,129.0,128.9,128.8,128.7,128.7,128.6,128.6,128.5$, 128.4, 128.4, 128.3, 128.2, 107.2 $\left(\mathrm{C}_{\beta}\right), 107.1\left(\mathrm{Cl}^{\mathrm{E}}\right), 106.2\left(\mathrm{C}^{\mathrm{G}}\right), 105.5\left(\mathrm{C}^{\mathrm{F}}\right), 101.3\left(\mathrm{C}_{\beta}\right), 100.9$ $\left(\mathrm{C1}^{\mathrm{B}}\right), 99.8\left(\mathrm{C1}^{\mathrm{D}}\right), 99.6\left(\mathrm{C1}^{\mathrm{C}}\right), 96.0\left(\mathrm{C}_{\beta}\right), 90.5\left(\mathrm{C} 1^{\mathrm{A}}\right), 82.7,82.5\left(\mathrm{C}_{\beta}\right), 82.3\left(\mathrm{C}_{\beta}\right), 82.3,82.0,81.9$, 81.8, 81.8 $\left(\mathrm{C}_{\beta}\right), 81.7\left(\mathrm{C}_{\beta}\right), 80.8,80.3\left(\mathrm{C}_{\beta}\right) 80.0\left(\mathrm{C}^{\mathrm{B}}\right)$, , 78.6, 78.2, 77.9, $77.8\left(\mathrm{C}_{\beta}\right), 76.0$ $\left(\mathrm{C}^{\mathrm{B}} / \mathrm{C}^{\mathrm{C}}\right), 75.9\left(\mathrm{C}_{\beta}\right), 75.7\left(\mathrm{C}^{\mathrm{B}} / \mathrm{C}^{\mathrm{C}}\right), 74.6\left(\mathrm{C}^{\mathrm{C}}\right), 74.2,74.0\left(\mathrm{C} 4^{\mathrm{A}} / \mathrm{C} 4^{\mathrm{B}}\right), 74.0,73.8\left(\mathrm{C} 4^{\mathrm{A}} / \mathrm{C}^{\mathrm{B}}\right)$, $73.2\left(\mathrm{C} 2^{\mathrm{C}}\right), 72.4,72.0\left(2 \mathrm{C}, \mathrm{C} 2^{\mathrm{A}}, \mathrm{C}^{\mathrm{D}}\right), 71.2\left(\mathrm{C} 2^{\mathrm{D}}\right), 70.4\left(\mathrm{C} 3^{\mathrm{A}}\right), 69.7\left(\mathrm{C} 4^{\mathrm{D}}\right), 64.4\left(\mathrm{C}_{\beta}\right), 64.3,64.1$, $63.9\left(\mathrm{C}^{\mathrm{E}}, \mathrm{C} 5^{\mathrm{F}}, \mathrm{C} 5^{\mathrm{G}}\right), 63.1\left(\mathrm{C}_{\beta}\right), 63.0\left(\mathrm{C} 5^{\mathrm{C}}\right), 62.9\left(\mathrm{C}^{\mathrm{D}}\right), 62.4\left(\mathrm{C}^{\mathrm{B}}\right), 59.4\left(\mathrm{C} 5^{\mathrm{A}}\right) . \mathrm{HRMS}: \mathrm{m} / z$ calcd for $\mathrm{C}_{140} \mathrm{H}_{118} \mathrm{O}_{44} \mathrm{~S}\left(\mathrm{Na}^{+}\right)_{2}[\mathrm{M}+2 \mathrm{Na}]^{2+} 1274.3390$, found 1274.3381 . 
2,3,4-Tri-O-benzoyl- $\beta$-D-xylopyranosyl-( $1 \rightarrow 4)$-[2,3,5-tri-O-benzoyl- $\alpha$-L-arabinofuranosyl$(1 \rightarrow 2)]$-[2,3,5-tri-O-benzoyl- $\alpha$-L-arabinofuranosyl-( $(1 \rightarrow 3)]$ - $\beta$-D-xylopyranosyl-( $1 \rightarrow 4)$-[2,3,5tri-O-benzoyl- $\alpha$-L-arabinofuranosyl-( $(1 \rightarrow 3)]$-2-O-benzoyl- $\beta$-D-xylopyranosyl- $(1 \rightarrow 4)-2,3$-di-Obenzoyl-D-xylopyranose (31). General procedure E with thioglycoside 28 (0.190 g, $0.073 \mathrm{mmol})$, acetone $/ \mathrm{H}_{2} \mathrm{O} 9: 1(1.1 \mathrm{~mL})$, NBS $(0.057 \mathrm{~g}, 0.32 \mathrm{mmol})$, additional NBS added after $30 \mathrm{~min}$ $(0.033 \mathrm{~g}, 0.19 \mathrm{mmol})$. Reaction time $60 \mathrm{~min}$. Eluent for TLC and flash column chromatography (heptane/acetone 3:2). Product was isolated as a white amorphous solid $(0.169 \mathrm{~g}, \alpha / \beta$ ratio $\sim 1: 0.3,92 \%) . R_{\mathrm{f}} 0.24$ (heptane/EtOAc 1:1). ${ }^{1} \mathrm{H}$ NMR $\left(400 \mathrm{MHz}, \mathrm{CDCl}_{3}\right) \delta 8.22(\mathrm{~d}, J=8.1 \mathrm{~Hz}$, 2H), 8.13-8.03 (m, 8H), 7.99-7.94 (m, 8H), 7.86-7.80 (m, 7H), 7.75-7.72 (m, 2H), 7.69 (d, $J=$ 7.4 Hz, 2H), 7.65-7.59 (m, 5H), 7.56-7.25 (m, 31H), 7.20-7.11 (m, 8H), 7.08-7.03 (m, 2H), $5.79\left(\mathrm{t}, J=9.5 \mathrm{~Hz}, 1 \mathrm{H}, \mathrm{H} 3^{\mathrm{A}}\right), 5.71\left(\mathrm{~s}, 1 \mathrm{H}, \mathrm{H}^{\mathrm{G}}\right), 5.64\left(\mathrm{t}, J=9.6 \mathrm{~Hz}, 1 \mathrm{H}, \mathrm{H} 3^{\mathrm{D}}\right), 5.58(\mathrm{~d}, J=2.7$ $\left.\mathrm{Hz}, 1 \mathrm{H}, \mathrm{H} 1^{\mathrm{F}}\right), 5.54(\mathrm{~s}, 1 \mathrm{H}), 5.53-5.49\left(\mathrm{~m}, 2 \mathrm{H}, \mathrm{H} 1^{\mathrm{A}}\right), 5.45-5.41(\mathrm{~m}, 2 \mathrm{H}), 5.37(\mathrm{t}, J=4.8 \mathrm{~Hz}, 2 \mathrm{H})$, $5.29(\mathrm{~s}, 0.2 \mathrm{H}), 5.21-5.19\left(\mathrm{~m}, 3 \mathrm{H}, \mathrm{H} 2^{\mathrm{D}}, \mathrm{H} 1^{\mathrm{E}}\right), 5.16-4.94\left(\mathrm{~m}, 6 \mathrm{H}, \mathrm{H} 2^{\mathrm{A}}, \mathrm{H} 2^{\mathrm{B}}, \mathrm{H} 4^{\mathrm{D}}\right), 4.87-4.83(\mathrm{~m}$, $3 \mathrm{H}), 4.81-4.75(\mathrm{~m}, 2 \mathrm{H}), 4.71\left(\mathrm{~d}, J=7.7 \mathrm{~Hz}, 1 \mathrm{H}, \mathrm{H1}^{\mathrm{A}}{ }\right), 4.38\left(\mathrm{~d}, J_{1 \mathrm{~B}, 2 \mathrm{~B}}=6.5 \mathrm{~Hz}, 1 \mathrm{H}, \mathrm{H} 1^{\mathrm{B}}\right), 4.35$ (d, $J=6.6 \mathrm{~Hz}, 0.3 \mathrm{H}), 3.98-3.92\left(\mathrm{~m}, 2 \mathrm{H}, \mathrm{H}^{\mathrm{B}}, \mathrm{H}^{\mathrm{D}}\right), 3.84-3.79\left(\mathrm{~m}, 2 \mathrm{H}, \mathrm{H} 1^{\mathrm{C}}, \mathrm{H} 3^{\mathrm{C}}\right), 3.76(\mathrm{t}, J=$ $\left.11.0 \mathrm{~Hz}, 1 \mathrm{H}, \mathrm{H} 5^{\mathrm{A}}\right), 3.62-3.30\left(\mathrm{~m}, 7 \mathrm{H}, \mathrm{H}^{\mathrm{A}}{ }^{\mathrm{A}}, \mathrm{H} 5^{\mathrm{A}}{ }^{\mathrm{A}}, \mathrm{H} 4^{\mathrm{B}}, \mathrm{H} 5^{\mathrm{B}}, \mathrm{H}^{\mathrm{C}}, \mathrm{H} 4^{\mathrm{C}}, \mathrm{H} 5^{\mathrm{D}}\right), 3.23(\mathrm{t}, J=11.2$ $\mathrm{Hz}, 0.4 \mathrm{H}), 3.10-2.96\left(\mathrm{~m}, 3 \mathrm{H}, \mathrm{H} 5^{\circ}{ }^{\mathrm{B}}, \mathrm{H} 5^{\mathrm{C}}, \mathrm{H} 5^{\circ} \mathrm{D}\right), 2.49-2.44\left(\mathrm{~m}, 1 \mathrm{H}, \mathrm{H} 5^{\circ} \mathrm{C}\right) .{ }^{13} \mathrm{C}\left\{{ }^{1} \mathrm{H}\right\} \mathrm{NMR}(101$ $\left.\mathrm{MHz}, \mathrm{CDCl}_{3}\right) \delta 167.1,166.6(\times 2), 166.4,166.3,166.0,165.9,165.8,165.8,165.8,165.8,165.6$, $165.5,165.5,165.4,165.4,165.0,165.0,164.9,164.9,164.9,164.4,164.4,134.0,133.5,133.5$, $133.5,133.4,133.4,133.3,133.3,133.1,133.0,132.9,132.9,132.9,130.3,130.2,130.1,130.1$, $130.0,129.9,129.9,129.9,129.8,129.8,129.7,129.7,129.6,129.4,129.3,129.2,129.1,129.0$, $129.0,128.9,128.9,128.7,128.6,128.6,128.5,128.4,128.3,128.3,128.3,128.2,106.1\left(\mathrm{C1}^{\mathrm{F}}\right)$, 105.7 $\left(\mathrm{C1}^{\mathrm{G}}\right), 105.4\left(\mathrm{C1}^{\mathrm{E}}\right), 105.3\left(\mathrm{C}_{\beta}\right), 100.2\left(\mathrm{C}_{\beta}\right), 100.1\left(\mathrm{C} 1^{\mathrm{B}}\right), 99.8\left(\mathrm{C1}^{\mathrm{D}}\right), 99.6\left(\mathrm{C1}^{\mathrm{C}}\right), 96.3$ 
$\left(\mathrm{C}_{\beta}\right), 90.5\left(\mathrm{C} 1^{\mathrm{A}}\right), 82.0,82.0,81.9,81.8,81.8\left(\mathrm{C}_{\beta}\right), 81.7,81.5,79.0,78.6,78.3,77.4\left(\mathrm{C} 2^{\mathrm{C}}\right), 76.7$

$\left(\mathrm{C} 3^{\mathrm{C}}\right), 75.4\left(\mathrm{C}^{\mathrm{B}}\right), 74.7,74.5\left(\mathrm{C}^{\mathrm{A}}, \mathrm{C}^{\mathrm{C}}\right), 74.3\left(\mathrm{C}_{\beta}\right), 74.2\left(\mathrm{C}_{\beta}\right), 73.1,73.1\left(\mathrm{C} 2^{\mathrm{B}}, \mathrm{C}^{\mathrm{B}}\right), 72.4,72.3$

$\left(\mathrm{C} 2^{\mathrm{A}}, \mathrm{C}^{\mathrm{D}}\right), 71.1\left(\mathrm{C} 2^{\mathrm{B}}\right), 70.1\left(\mathrm{C} 3^{\mathrm{C}}\right), 69.7\left(\mathrm{C}^{\mathrm{D}}\right), 64.3,64.1,64.1\left(\mathrm{C} 5^{\mathrm{E}}, \mathrm{C} 5^{\mathrm{F}}, \mathrm{C} 5^{\mathrm{G}}\right), 63.5\left(\mathrm{C}_{\beta}\right)$,

63.0, $62.8\left(\mathrm{C}^{\mathrm{C}}, \mathrm{C}^{\mathrm{D}}\right), 62.0\left(\mathrm{C}^{\mathrm{B}}\right), 59.3\left(\mathrm{C}^{\mathrm{A}}\right)$. HRMS: $m / z$ calcd for $\mathrm{C}_{140} \mathrm{H}_{118} \mathrm{O}_{44} \mathrm{~S}\left(\mathrm{Na}^{+}\right)_{2}[\mathrm{M}+$ $2 \mathrm{Na}]^{2+} 1274.3390$, found 1274.3378 .

Phenyl $\beta$-D-xylopyranosyl- $(1 \rightarrow 4)$-[ $\alpha$-L-arabinofuranosyl- $(1 \rightarrow 3)]$ - $\beta$-D-xylopyranosyl- $(1 \rightarrow 4)-[\alpha$ -

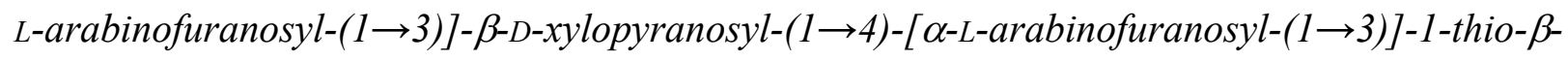
D-xylopyranoside (32). General procedure F with heptasaccharide 19 (0.028 g, $0.011 \mathrm{mmol})$. The product was obtained as a white powder $(0.009 \mathrm{~g}, 77 \%) . R_{\mathrm{f}} 0.63$ (EtOAc/MeOH/H$/ \mathrm{H}_{2} \mathrm{O} / \mathrm{AcOH}$ 6:3:0.8:0.2). $[\alpha]^{25} \mathrm{D}-30\left(c\right.$ 0.23, $\left.\mathrm{H}_{2} \mathrm{O}\right) .{ }^{1} \mathrm{H}$ NMR $\left(800 \mathrm{MHz}, \mathrm{D}_{2} \mathrm{O}\right) \delta$ 7.60-7.57 (m, 2H), 7.47-7.43 (m, 3H), $5.43\left(\mathrm{~s}, 1 \mathrm{H}, \mathrm{H} 1^{\text {arabinose }}\right), 5.41\left(\mathrm{~s}, 1 \mathrm{H}, \mathrm{H} 1^{\text {arabinose }}\right), 5.40(\mathrm{~s}$, $\left.1 \mathrm{H}, \mathrm{H} 1^{\text {arabinose }}\right), 4.82\left(\mathrm{~d}, J=9.6 \mathrm{~Hz}, 1 \mathrm{H}, \mathrm{H} 1^{\mathrm{A}}\right), 4.49-4.48\left(\mathrm{~m}, 2 \mathrm{H}, \mathrm{H} 1^{\mathrm{B}}, \mathrm{H} 1^{\mathrm{C}}\right), 4.45(\mathrm{~d}, J=7.8 \mathrm{~Hz}$, 1H, H1 $\left.{ }^{\mathrm{D}}\right), 4.30-4.27$ (m, 3H, H4 $\left.{ }^{\text {arabinose }}\right), 4.19-4.17$ (m, 4H, H5 $\left.{ }^{\mathrm{A}}, \mathrm{H} 2^{\text {arabinose }}\right), 4.10-4.06$ (m, 2H, $\left.\mathrm{H}^{\mathrm{B}}, \mathrm{H} 5^{\mathrm{C}}\right), 3.93-3.91\left(\mathrm{~m}, 4 \mathrm{H}, \mathrm{H} 5^{\mathrm{D}}, \mathrm{H} 3^{\text {arabinose }}\right), 3.82-3.79\left(\mathrm{~m}, 7 \mathrm{H}, \mathrm{H} 3^{\mathrm{A}}, \mathrm{H}^{\mathrm{A}}, \mathrm{H} 4^{\mathrm{B}}, \mathrm{H}^{\mathrm{C}}\right.$, H5 $\left.{ }^{\text {arabinose }}\right), 3.76-3.72\left(\mathrm{~m}, 5 \mathrm{H}, \mathrm{H}^{\mathrm{B}}{ }^{\mathrm{H}}, \mathrm{H} 3^{\mathrm{C}}, \mathrm{H} 5^{\text {'arabinose }}\right), 3.61$ (td, $\left.J=9.9,5.5 \mathrm{~Hz}, 1 \mathrm{H}, \mathrm{H}^{\mathrm{D}}\right), 3.53$ (t, $\left.J=8.8 \mathrm{~Hz}, 1 \mathrm{H}, \mathrm{H} 2^{\mathrm{A}}\right), 3.46-3.41\left(\mathrm{~m}, 4 \mathrm{H}, \mathrm{H} 5^{\prime}{ }^{\mathrm{A}}, \mathrm{H}^{\mathrm{B}}, \mathrm{H} 2^{\mathrm{C}}, \mathrm{H} 3^{\mathrm{D}}\right), 3.37\left(\mathrm{t}, J=11.1 \mathrm{~Hz}, 2 \mathrm{H}, \mathrm{H} 5^{\circ}{ }^{\mathrm{B}}\right.$, $\left.\mathrm{H} 5^{, \mathrm{C}}\right), 3.28\left(\mathrm{t}, J=11.1 \mathrm{~Hz}, 1 \mathrm{H}, \mathrm{H} 5^{\circ} \mathrm{D}\right), 3.26-3.24\left(\mathrm{~m}, 1 \mathrm{H}, \mathrm{H} 2^{\mathrm{D}}\right) .{ }^{13} \mathrm{C}\left\{{ }^{1} \mathrm{H}\right\} \mathrm{NMR}\left(201 \mathrm{MHz}, \mathrm{D}_{2} \mathrm{O}\right)$ $\delta 132.4(\times 2), 131.2,129.4(\times 2), 128.4,107.7,107.6,107.6\left(\mathrm{C}^{\text {arabinose }}\right), 101.4\left(\mathrm{C}^{\mathrm{D}}\right), 101.2$, 101.2 $\left(\mathrm{C}^{\mathrm{B}}, \mathrm{C}^{\mathrm{C}}\right), 87.8\left(\mathrm{C}^{\mathrm{A}}\right), 84.8,84.7,84.7\left(\mathrm{C} 4^{\text {arabinose }}\right), 80.7,80.7,80.7\left(\mathrm{C}^{\text {arabinose }}\right), 78.7$ $\left(\mathrm{C} 3^{\mathrm{A}}\right), 77.3,77.2,77.2,77.2\left(\mathrm{C}^{\mathrm{B}}, \mathrm{C}^{\mathrm{C}}, \mathrm{C}^{\text {arabinose }}\right), 75.6\left(\mathrm{C} 3^{\mathrm{D}}\right), 73.6,73.6,73.5,73.5,73.5\left(\mathrm{C}^{\mathrm{A}}\right.$, $\left.\mathrm{C} 2^{\mathrm{B}}, \mathrm{C} 4^{\mathrm{B}}, \mathrm{C} 2^{\mathrm{C}}, \mathrm{C} 4^{\mathrm{C}}\right), 72.9\left(\mathrm{C} 2^{\mathrm{D}}\right), 71.9\left(\mathrm{C} 2^{\mathrm{A}}\right), 69.2\left(\mathrm{C}^{\mathrm{D}}\right), 66.3\left(\mathrm{C} 5^{\mathrm{A}}\right), 65.1\left(\mathrm{C} 5^{\mathrm{D}}\right), 62.7,62.7$ 
$\left(\mathrm{C}^{\mathrm{B}}, \mathrm{C}^{\mathrm{C}}\right), 61.3$ (C5 $\left.5^{\text {arabinose }}\right)$. HRMS: $m / z$ calcd for $\mathrm{C}_{41} \mathrm{H}_{63} \mathrm{O}_{28} \mathrm{~S}[\mathrm{M}+\mathrm{H}]^{+}$1035.3221, found 1035.3214.

Phenyl $\beta$-D-xylopyranosyl- $(1 \rightarrow 4)$-[ $\alpha$-L-arabinofuranosyl- $(1 \rightarrow 3)]-\beta$-D-xylopyranosyl- $(1 \rightarrow 4)-[\alpha$ L-arabinofuranosyl- $(1 \rightarrow 2)]$-[ $\alpha$-L-arabinofuranosyl- $(1 \rightarrow 3)]$ - $\beta$-D-xylopyranosyl-(1 $\rightarrow 4)$-1-thio- $\beta$ D-xylopyranoside (33). General procedure F with heptasaccharide 23 (0.071 g, $0.027 \mathrm{mmol})$. The product was obtained as a white powder $(0.023 \mathrm{~g}, 82 \%) . R_{\mathrm{f}} 0.53$

(EtOAc/MeOH/ $\mathrm{H}_{2} \mathrm{O} / \mathrm{AcOH}$ 6:3:0.8:0.2). $[\alpha]^{25} \mathrm{D}-36\left(c\right.$ 0.21, $\left.\mathrm{H}_{2} \mathrm{O}\right) .{ }^{1} \mathrm{H}$ NMR $\left(800 \mathrm{MHz}, \mathrm{D}_{2} \mathrm{O}\right) \delta$ 7.59-7.58 (m, 2H), 7.46-7.42 (m, 3H), $5.42\left(\mathrm{~s}, 1 \mathrm{H}, \mathrm{H} 1^{\mathrm{G}}\right), 5.28\left(\mathrm{~s}, 1 \mathrm{H}, \mathrm{H} 1^{\mathrm{F}}\right), 5.23\left(\mathrm{~s}, 1 \mathrm{H}, \mathrm{H} 1^{\mathrm{E}}\right)$, $4.77\left(\mathrm{~d}, J=9.5 \mathrm{~Hz}, 1 \mathrm{H}, \mathrm{H}^{\mathrm{A}}\right), 4.64\left(\mathrm{~d}, J=7.2 \mathrm{~Hz}, 1 \mathrm{H}, \mathrm{H}^{\mathrm{B}}\right), 4.49\left(\mathrm{~d}, J=7.9 \mathrm{~Hz}, 1 \mathrm{H}, \mathrm{H} 1^{\mathrm{C}}\right), 4.45$ $\left(\mathrm{d}, J=7.8 \mathrm{~Hz}, 1 \mathrm{H}, \mathrm{H}^{\mathrm{D}}\right), 4.32\left(\mathrm{q}, J=5.4 \mathrm{~Hz}, 1 \mathrm{H}, \mathrm{H}^{\mathrm{F}}\right), 4.29\left(\mathrm{q}, J=5.2 \mathrm{~Hz}, 1 \mathrm{H}, \mathrm{H}^{\mathrm{G}}\right), 4.19-4.14$ $\left(\mathrm{m}, 5 \mathrm{H}, \mathrm{H} 5^{\mathrm{A}}, \mathrm{H}^{\mathrm{B}}, \mathrm{H} 2^{\mathrm{E}}, \mathrm{H} 2^{\mathrm{F}}, \mathrm{H} 2^{\mathrm{G}}\right), 4.12\left(\mathrm{td}, J=5.8,3.5 \mathrm{~Hz}, 1 \mathrm{H}, \mathrm{H} 4^{\mathrm{E}}\right), 4.09(\mathrm{dd}, J=11.8,5.2$ Hz, 1H, H5 $\left.5^{\mathrm{C}}\right), 3.96-3.95\left(\mathrm{~m}, 2 \mathrm{H}, \mathrm{H}^{\mathrm{E}}, \mathrm{H} 3^{\mathrm{F}}\right), 3.94-3.91\left(\mathrm{~m}, 2 \mathrm{H}, \mathrm{H} 5^{\mathrm{D}}, \mathrm{H} 3^{\mathrm{G}}\right), 3.89$ (td, $J=9.0,5.1$ $\left.\mathrm{Hz}, 1 \mathrm{H}, \mathrm{H} 4^{\mathrm{B}}\right), 3.84-3.77\left(\mathrm{~m}, 6 \mathrm{H}, \mathrm{H} 4^{\mathrm{A}}, \mathrm{H} 3^{\mathrm{B}}, \mathrm{H} 4^{\mathrm{C}}, \mathrm{H} 5^{\mathrm{E}}, \mathrm{H} 5^{\mathrm{F}}, \mathrm{H} 5^{\mathrm{G}}\right), 3.77-3.70\left(\mathrm{~m}, 4 \mathrm{H}, \mathrm{H} 3^{\mathrm{C}}\right.$, $\left.\mathrm{H}^{\circ}{ }^{\mathrm{E}}, \mathrm{H} 5^{\mathrm{F}}{ }^{\mathrm{F}}, \mathrm{H} 5^{\circ}{ }^{\mathrm{G}}\right), 3.63-3.59\left(\mathrm{~m}, 2 \mathrm{H}, \mathrm{H}^{\mathrm{A}}{ }^{2}, \mathrm{H} 4^{\mathrm{D}}\right), 3.59-3.57\left(\mathrm{~m}, 1 \mathrm{H}, \mathrm{H} 2^{\mathrm{B}}\right), 3.47-3.36(\mathrm{~m}, 6 \mathrm{H}$, $\left.\mathrm{H} 2^{\mathrm{A}}, \mathrm{H} 5^{\prime}{ }^{\mathrm{A}}, \mathrm{H} 5^{\prime \mathrm{B}}, \mathrm{H}^{\mathrm{C}}{ }^{2}, \mathrm{H} 5^{\prime}{ }^{\mathrm{C}}, \mathrm{H}^{\mathrm{D}}\right), 3.29\left(\mathrm{t}, J=11.4 \mathrm{~Hz}, 1 \mathrm{H}, \mathrm{H} 5^{\circ} \mathrm{D}\right), 3.26(\mathrm{dd}, J=9.2,8.0 \mathrm{~Hz}$, $\left.1 \mathrm{H}, \mathrm{H} 2{ }^{\mathrm{D}}\right) .{ }^{13} \mathrm{C}\left\{{ }^{1} \mathrm{H}\right\}$ NMR $\left(201 \mathrm{MHz}, \mathrm{D}_{2} \mathrm{O}\right) \delta 132.1(\times 2), 131.4,129.4(\times 2), 128.3,108.7\left(\mathrm{C}^{\mathrm{E}}\right)$, $108.1\left(\mathrm{C}^{\mathrm{F}}\right), 107.6\left(\mathrm{C}^{\mathrm{G}}\right), 101.4\left(\mathrm{C}^{\mathrm{D}}\right), 101.2\left(\mathrm{C}^{\mathrm{C}}\right), 99.8\left(\mathrm{C1}^{\mathrm{B}}\right), 88.0\left(\mathrm{C} 1^{\mathrm{A}}\right), 84.8\left(\mathrm{C} 4^{\mathrm{G}}\right), 84.4$ $\left(\mathrm{C} 4^{\mathrm{E}}\right), 84.2\left(\mathrm{C}^{\mathrm{F}}\right), 81.2,81.0,80.7\left(\mathrm{C} 2^{\mathrm{E}}, \mathrm{C} 2^{\mathrm{F}}, \mathrm{C} 2^{\mathrm{G}}\right), 78.6\left(\mathrm{C} 2^{\mathrm{B}}\right), 77.6\left(\mathrm{C} 3^{\mathrm{B}}\right), 77.2,77.2,77.2$ $\left(\mathrm{C}^{\mathrm{C}}, \mathrm{C}^{\mathrm{F}}, \mathrm{C}^{\mathrm{G}}\right), 76.6\left(\mathrm{C}^{\mathrm{E}}\right), 75.6\left(\mathrm{C}^{\mathrm{D}}\right), 75.4\left(\mathrm{C}^{\mathrm{A}}\right), 75.2\left(\mathrm{C} 3^{\mathrm{A}}\right), 73.8,73.7,73.6\left(\mathrm{C}^{\mathrm{B}}, \mathrm{C}^{\mathrm{C}}\right.$, $\left.\mathrm{C}^{\mathrm{C}}\right), 72.9\left(\mathrm{C}^{\mathrm{D}}\right), 71.6\left(\mathrm{C} 2^{\mathrm{A}}\right), 69.2\left(\mathrm{C}^{\mathrm{D}}\right), 66.5\left(\mathrm{C} 5^{\mathrm{A}}\right), 65.1\left(\mathrm{C}^{\mathrm{D}}\right), 62.7\left(\mathrm{C}^{\mathrm{C}}\right), 62.5\left(\mathrm{C} 5^{\mathrm{B}}\right), 61.3$, 61.2, $61.1\left(\mathrm{C}^{\mathrm{E}}, \mathrm{C}^{\mathrm{F}}, \mathrm{C}^{\mathrm{G}}\right)$. HRMS: $m / z$ calcd for $\mathrm{C}_{41} \mathrm{H}_{63} \mathrm{O}_{28} \mathrm{~S}[\mathrm{M}+\mathrm{H}]^{+}$1035.3221, found 1035.3216. 
Phenyl $\beta$-D-xylopyranosyl-( $(1 \rightarrow 4)$-[ $\alpha$-L-arabinofuranosyl- $(1 \rightarrow 2)]$-[ $\alpha$-L-arabinofuranosyl$(1 \rightarrow 3)]$ - $\beta$-D-xylopyranosyl-( $(1 \rightarrow 4)$-[ $\alpha$-L-arabinofuranosyl-( $(1 \rightarrow 3)]$ - $\beta$-D-xylopyranosyl- $(1 \rightarrow 4)$ - 1 thio- $\beta$-D-xylopyranoside (34). General procedure F with heptasaccharide 28 (0.061 g, 0.024 mmol). Product was obtained as a white powder $(0.022 \mathrm{~g}, 91 \%) . R_{\mathrm{f}} 0.53$ (EtOAc/MeOH/ $\mathrm{H}_{2} \mathrm{O} / \mathrm{AcOH}$ 6:3:0.8:0.2). $[\alpha]^{25} \mathrm{D}-22\left(c 0.28, \mathrm{H}_{2} \mathrm{O}\right) .{ }^{1} \mathrm{H}$ NMR $\left(800 \mathrm{MHz}, \mathrm{D}_{2} \mathrm{O}\right) \delta$ 7.59-7.58 (m, 2H), 7.46-7.43 (m, 3H), $5.43\left(\mathrm{~s}, 1 \mathrm{H}, \mathrm{H} 1^{\mathrm{E}}\right), 5.29\left(\mathrm{~s}, 1 \mathrm{H}, \mathrm{H} 1^{\mathrm{G}}\right), 5.25\left(\mathrm{~s}, 1 \mathrm{H}, \mathrm{H} 1^{\mathrm{F}}\right)$, $4.79\left(\mathrm{~d}, J=9.2 \mathrm{~Hz}, 1 \mathrm{H}, \mathrm{H} 1^{\mathrm{A}}\right), 4.61\left(\mathrm{~d}, J=7.5 \mathrm{~Hz}, 1 \mathrm{H}, \mathrm{H} 1^{\mathrm{C}}\right), 4.50\left(\mathrm{~d}, J=7.7 \mathrm{~Hz}, 1 \mathrm{H}, \mathrm{H} 1^{\mathrm{B}}\right), 4.44$ $\left(\mathrm{d}, J=7.8 \mathrm{~Hz}, 1 \mathrm{H}, \mathrm{H} 1^{\mathrm{D}}\right), 4.34-4.31\left(\mathrm{~m}, 2 \mathrm{H}, \mathrm{H} 4^{\mathrm{E}}, \mathrm{H} 4^{\mathrm{G}}\right), 4.18-4.13\left(\mathrm{~m}, 6 \mathrm{H}, \mathrm{H} 5^{\mathrm{A}}, \mathrm{H}^{\mathrm{B}}, \mathrm{H} 2^{\mathrm{E}}, \mathrm{H} 2^{\mathrm{F}}\right.$, $\left.\mathrm{H} 4^{\mathrm{F}}, \mathrm{H} 2^{\mathrm{G}}\right), 4.10\left(\mathrm{dd}, J=11.9,4.4 \mathrm{~Hz}, 1 \mathrm{H}, \mathrm{H} 5^{\mathrm{C}}\right), 3.99\left(\mathrm{dd}, J=5.8,3.1 \mathrm{~Hz}, 1 \mathrm{H}, \mathrm{H} 3^{\mathrm{F}}\right), 3.95-3.92$ (m, 3H, H5 $5^{\mathrm{D}}, \mathrm{H}^{\mathrm{E}}, \mathrm{H}^{\mathrm{G}}$ ), 3.86-3.73 (m, 11H, H4 ${ }^{\mathrm{A}}, \mathrm{H} 3^{\mathrm{B}}, \mathrm{H} 4^{\mathrm{B}}, \mathrm{H} 3^{\mathrm{C}}, \mathrm{H} 4^{\mathrm{C}}, \mathrm{H} 5^{\mathrm{E}}, \mathrm{H} 5^{\mathrm{E}}, \mathrm{H} 5^{\mathrm{F}}, \mathrm{H} 5^{\mathrm{F}}$, $\left.\mathrm{H}^{\mathrm{G}}, \mathrm{H} 5^{\circ} \mathrm{G}\right), 3.63-3.60\left(\mathrm{~m}, 2 \mathrm{H}, \mathrm{H}^{\mathrm{A}}, \mathrm{H}^{\mathrm{D}}\right), 3.57\left(\mathrm{t}, J=7.8 \mathrm{~Hz}, 1 \mathrm{H} \mathrm{H} 2^{\mathrm{C}}\right), 3.48-3.38\left(\mathrm{~m}, 6 \mathrm{H}, \mathrm{H} 2^{\mathrm{A}}\right.$,

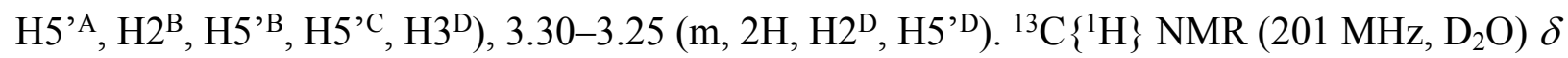
$132.1(\times 2), 131.4,129.4(\times 2), 128.4,108.7\left(\mathrm{C} 1^{\mathrm{F}}\right), 108.0\left(\mathrm{C} 1^{\mathrm{G}}\right), 107.5\left(\mathrm{C} 1^{\mathrm{E}}\right), 101.6\left(\mathrm{C} 1^{\mathrm{B}}\right), 101.3$ $\left(\mathrm{C}^{\mathrm{D}}\right), 99.8\left(\mathrm{C}^{\mathrm{C}}\right), 87.9\left(\mathrm{C} 1^{\mathrm{A}}\right), 85.0\left(\mathrm{C} 4^{\mathrm{E}} / \mathrm{C} 4^{\mathrm{G}}\right), 84.3\left(\mathrm{C} 4^{\mathrm{F}}\right), 84.2\left(\mathrm{C} 4^{\mathrm{E}} / \mathrm{C} 4^{\mathrm{G}}\right), 81.3\left(\mathrm{C} 2^{\mathrm{F}}\right), 80.9$, $80.6\left(\mathrm{C}^{\mathrm{E}}, \mathrm{C} 2^{\mathrm{G}}\right), 78.7\left(\mathrm{C} 2^{\mathrm{C}}\right), 77.7\left(\mathrm{C}^{\mathrm{C}}\right), 77.2,77.2\left(\mathrm{C}^{\mathrm{E}}, \mathrm{C}^{\mathrm{G}}\right), 77.0\left(\mathrm{C} 3^{\mathrm{B}}\right), 76.7\left(\mathrm{C} 3^{\mathrm{F}}\right), 76.1$ $\left(\mathrm{C} 4^{\mathrm{A}}\right), 75.6\left(\mathrm{C}^{\mathrm{D}}\right), 75.2\left(\mathrm{C}^{\mathrm{A}}\right), 73.6,73.3\left(\mathrm{C} 4^{\mathrm{B}}, \mathrm{C} 4^{\mathrm{C}}\right), 73.3\left(\mathrm{C}^{\mathrm{B}}\right), 73.0\left(\mathrm{C} 2^{\mathrm{D}}\right), 71.6\left(\mathrm{C} 2^{\mathrm{A}}\right), 69.2$ $\left(\mathrm{C} 4^{\mathrm{D}}\right), 66.5\left(\mathrm{C}^{\mathrm{A}}\right), 65.1\left(\mathrm{C}^{\mathrm{D}}\right), 62.8\left(\mathrm{C}^{\mathrm{B}}\right), 62.6\left(\mathrm{C5}^{\mathrm{C}}\right), 61.3,61.1,61.1\left(\mathrm{C}^{\mathrm{E}}, \mathrm{C5}^{\mathrm{F}}, \mathrm{C} 5^{\mathrm{G}}\right)$. HRMS: $m / z$ calcd for $\mathrm{C}_{41} \mathrm{H}_{63} \mathrm{O}_{28} \mathrm{~S}[\mathrm{M}+\mathrm{H}]^{+} 1035.3221$, found 1035.3218 .

$\beta$-D-Xylopyranosyl-( $(1 \rightarrow 4)$-[ $\alpha$-L-arabinofuranosyl-( $(1 \rightarrow 3)]-\beta$-D-xylopyranosyl- $(1 \rightarrow 4)-[\alpha-L$ arabinofuranosyl-( $(1 \rightarrow 3)]$ - $\beta$-D-xylopyranosyl- $(1 \rightarrow 4)$-[ $\alpha$-L-arabinofuranosyl- $(1 \rightarrow 3)]-D$ xylopyranose (1). General procedure G with partially deprotected heptasaccharide 32 (10.0 mg, 
$0.097 \mathrm{mmol}$ ), 2,6-lutidine ( $3 \mu \mathrm{L}, 0.015 \mathrm{mmol}$ ) and NBS (5.3 mg, $0.030 \mathrm{mmol})$. Reaction time $1.5 \mathrm{~h}$. The product was obtained as a white powder $(8.8 \mathrm{mg}, 97 \%) . \alpha / \beta$ ratio $0.7: 1 . R_{\mathrm{f}} 0.45$ (EtOAc/MeOH/H $\mathrm{H}_{2} \mathrm{O} / \mathrm{AcOH}$ 6:3:0.8:0.2). ${ }^{1} \mathrm{H}$ NMR (800 MHz, $\left.\mathrm{D}_{2} \mathrm{O}\right) \delta 5.41-5.41(\mathrm{~m}, 4.01 \mathrm{H}$ $\left.\mathrm{H} 1^{\mathrm{F}}, \mathrm{H} 1^{\mathrm{G}}\right), 5.36\left(\mathrm{~s}, 0.73 \mathrm{H}, \mathrm{H} 1^{\mathrm{E}}\right), 5.19\left(\mathrm{~d}, J=3.5 \mathrm{~Hz}, 0.70 \mathrm{H}, \mathrm{H}^{\mathrm{A}}{ }_{\alpha}\right), 4.64(\mathrm{~d}, J=7.9 \mathrm{~Hz}, 1 \mathrm{H}$, $\left.\mathrm{H}^{\mathrm{A}}{ }_{\beta}\right), 4.51-4.49\left(\mathrm{~m}, 3.35 \mathrm{H}, \mathrm{H}^{\mathrm{B}}, \mathrm{H} 1^{\mathrm{C}}\right), 4.45-4.44\left(\mathrm{~m}, 1.76 \mathrm{H}, \mathrm{H} 1^{\mathrm{D}}\right), 4.31-4.28\left(\mathrm{~m}, 4.90 \mathrm{H}, \mathrm{H}^{\mathrm{E}}\right.$, $\left.\mathrm{H} 4^{\mathrm{F}}, \mathrm{H} 4^{\mathrm{G}}\right), 4.19-4.17\left(\mathrm{~m}, 4.94 \mathrm{H}, \mathrm{H} 2^{\mathrm{E}}, \mathrm{H} 2^{\mathrm{F}}, \mathrm{H} 2^{\mathrm{G}}\right), 4.10-4.07\left(\mathrm{~m}, 4.29 \mathrm{H}, \mathrm{H} 5^{\mathrm{A}}{ }_{\beta}, \mathrm{H} 5^{\mathrm{B}}, \mathrm{H} 5^{\mathrm{C}}\right)$, 3.94-3.91 (m, 7.29H, H3 $\left.{ }_{\alpha}^{\mathrm{A}}, \mathrm{H}^{\mathrm{D}}, \mathrm{H} 3^{\mathrm{E}}, \mathrm{H} 3^{\mathrm{F}}, \mathrm{H} 3^{\mathrm{G}}\right), 3.87-3.78\left(\mathrm{~m}, 12.26 \mathrm{H}, \mathrm{H} 4^{\mathrm{A}}{ }_{\alpha}, \mathrm{H} 4^{\mathrm{A}}{ }_{\beta}, \mathrm{H}^{\mathrm{A}}{ }_{\alpha}\right.$, $\left.\mathrm{H}^{\prime}{ }_{\alpha}^{\mathrm{A}}, \mathrm{H}^{\mathrm{B}}, \mathrm{H} 5^{\mathrm{E}}, \mathrm{H} 5^{\mathrm{F}}, \mathrm{H} 5^{\mathrm{G}}\right), 3.76-3.72\left(\mathrm{~m}, 9.69 \mathrm{H}, \mathrm{H} 3^{\mathrm{A}}{ }_{\beta}, \mathrm{H} 3^{\mathrm{B}}, \mathrm{H} 3^{\mathrm{C}}, \mathrm{H} 5^{\circ}{ }^{\mathrm{E}}, \mathrm{H} 5^{\circ}{ }^{\mathrm{F}}, \mathrm{H} 5^{\prime} \mathrm{G}\right), 3.70$ $\left(\mathrm{dd}, J=9.2,3.6 \mathrm{~Hz}, 1.06 \mathrm{H}, \mathrm{H} 2^{\mathrm{A}}{ }_{\alpha}\right), 3.63-3.59\left(\mathrm{~m}, 2 \mathrm{H}, \mathrm{H} 4^{\mathrm{D}}\right), 3.47-3.36\left(\mathrm{~m}, 10.88 \mathrm{H}, \mathrm{H} 2^{\mathrm{A}}{ }_{\beta}, \mathrm{H} 5^{\mathrm{A}}{ }_{\beta}\right.$, $\left.\mathrm{H} 2^{\mathrm{B}}, \mathrm{H} 5^{\circ}{ }^{\mathrm{B}}, \mathrm{H} 2^{\mathrm{C}}, \mathrm{H} 5^{{ }^{\mathrm{C}}}, \mathrm{H} 3^{\mathrm{D}}\right), 3.30-3.24\left(\mathrm{~m}, 3.99 \mathrm{H}, \mathrm{H} 2^{\mathrm{D}}, \mathrm{H} 5^{, \mathrm{D}}\right) .{ }^{13} \mathrm{C}\left\{{ }^{1} \mathrm{H}\right\} \mathrm{NMR}\left(201 \mathrm{MHz}, \mathrm{D}_{2} \mathrm{O}\right)$ $\delta 107.8,107.7,107.7,107.6\left(\mathrm{C1}^{\mathrm{E}}, \mathrm{C} 1^{\mathrm{F}}, \mathrm{C} 1^{\mathrm{G}}\right), 101.4,101.3,101.3,101.2\left(\mathrm{C}^{\mathrm{B}}, \mathrm{C1}^{\mathrm{C}}, \mathrm{C} 1^{\mathrm{D}}\right), 96.4$ $\left(\mathrm{C}^{\mathrm{A}}{ }_{\beta}\right), 92.2\left(\mathrm{C1}_{\alpha}^{\mathrm{A}}\right), 84.8,84.7,84.7,84.6\left(\mathrm{C} 4^{\mathrm{E}}, \mathrm{C} 4^{\mathrm{F}}, \mathrm{C} 4^{\mathrm{G}}\right), 80.7,80.7,80.7\left(\mathrm{C} 2^{\mathrm{E}}, \mathrm{C} 2^{\mathrm{F}}, \mathrm{C} 2^{\mathrm{G}}\right)$, $77.7\left(\mathrm{C}^{\mathrm{A}}{ }_{\beta}\right), 77.3,77.3,77.2,77.2\left(\mathrm{C}^{\mathrm{B}}, \mathrm{C} 3^{\mathrm{C}}, \mathrm{C} 3^{\mathrm{E}}, \mathrm{C} 3^{\mathrm{F}}, \mathrm{C} 3^{\mathrm{G}}\right), 75.6\left(\mathrm{C} 3^{\mathrm{D}}\right), 75.2\left(\mathrm{C}^{\mathrm{A}}{ }_{\alpha}\right), 74.5$ $\left(\mathrm{C} 2^{\mathrm{A}}{ }_{\beta}\right), 73.8,73.7,73.6,73.6,73.6,73.5,73.5\left(\mathrm{C} 4^{\mathrm{A}}{ }_{\alpha}, \mathrm{C} 4^{\mathrm{A}}{ }_{\beta}, \mathrm{C} 2^{\mathrm{B}}, \mathrm{C} 4^{\mathrm{B}}, \mathrm{C} 2^{\mathrm{C}}, \mathrm{C} 4^{\mathrm{C}}\right), 72.9\left(\mathrm{C} 2^{\mathrm{D}}\right)$, $71.7\left(\mathrm{C} 2^{\mathrm{A}}{ }_{\alpha}\right), 69.2\left(\mathrm{C}^{\mathrm{D}}\right), 65.1\left(\mathrm{C}^{\mathrm{D}}\right), 62.8,62.7,62.7,62.7\left(\mathrm{C} 5^{\mathrm{A}}{ }_{\beta}, \mathrm{C} 5^{\mathrm{B}}, \mathrm{C}^{\mathrm{C}}\right), 61.3\left(\mathrm{C}^{\mathrm{E}}, \mathrm{C}^{\mathrm{F}}\right.$, $\left.\mathrm{C}^{\mathrm{G}}\right), 59.1\left(\mathrm{C}^{\mathrm{A}}{ }_{\alpha}\right)$. HRMS: $m / z$ calcd for $\mathrm{C}_{35} \mathrm{H}_{58} \mathrm{O}_{29} \mathrm{Na}[\mathrm{M}+\mathrm{Na}]^{+}$965.2956, found 965.2962.

$\beta$-D-Xylopyranosyl-( $1 \rightarrow 4)$-[ $\alpha$-L-arabinofuranosyl- $(1 \rightarrow 3)]-\beta$-D-xylopyranosyl- $(1 \rightarrow 4)-[\alpha-L$ arabinofuranosyl-(1 $\rightarrow 2)]$-[ $\alpha$-L-arabinofuranosyl- $(1 \rightarrow 3)]-\beta$-D-xylopyranosyl- $(1 \rightarrow 4)$-Dxylopyranose (2). General procedure $\mathrm{G}$ with partially deprotected heptasaccharide 33 (13.4 mg, $0.013 \mathrm{mmol}$ ), 2,6-lutidine ( $4 \mu \mathrm{L}, 0.019 \mathrm{mmol})$ and NBS (6.8 mg, $0.038 \mathrm{mmol})$. Reaction time 1.5 h. The product was obtained as a white powder $(10.2 \mathrm{mg}, 84 \%) . \alpha / \beta$ ratio $0.5: 1 . R_{\mathrm{f}} 0.31$ 
(EtOAc/MeOH/H$/ \mathrm{H}_{2} \mathrm{O} / \mathrm{AcOH}$ 6:3:0.8:0.2). ${ }^{1} \mathrm{H}$ NMR (800 MHz, $\left.\mathrm{D}_{2} \mathrm{O}\right) \delta 5.43\left(\mathrm{~s}, 1.54 \mathrm{H}, \mathrm{H}^{\mathrm{G}}\right), 5.29$ $\left(\mathrm{s}, 1.40 \mathrm{H}, \mathrm{H} 1^{\mathrm{F}}\right), 5.25\left(\mathrm{~s}, 0.53 \mathrm{H}, \mathrm{H} 1^{\mathrm{E}}{ }_{\alpha / \beta}\right), 5.25\left(\mathrm{~s}, 0.86 \mathrm{H}, \mathrm{H} 1_{\alpha / \beta}^{\mathrm{E}}\right), 5.21(\mathrm{~d}, J=3.6 \mathrm{~Hz}, 0.53 \mathrm{H}$, $\left.\mathrm{H} 1_{\alpha}\right), 4.66\left(\mathrm{~d}, J=7.2 \mathrm{~Hz}, 1.37 \mathrm{H}, \mathrm{H} 1^{\mathrm{B}}\right), 4.60\left(\mathrm{~d}, J=7.9 \mathrm{~Hz}, 1 \mathrm{H}, \mathrm{H} 1_{\beta}^{\mathrm{A}}\right), 4.50(\mathrm{~d}, J=7.8 \mathrm{~Hz}$, $\left.1.43 \mathrm{H}, \mathrm{H} 1^{\mathrm{C}}\right), 4.46\left(\mathrm{~d}, J=7.8 \mathrm{~Hz}, 1.49 \mathrm{H}, \mathrm{H} 1^{\mathrm{D}}\right), 4.33\left(\mathrm{td}, J=5.4,3.8 \mathrm{~Hz}, 1.54 \mathrm{H}, \mathrm{H} 4^{\mathrm{F}}\right), 4.30(\mathrm{q}, J$ $\left.=5.3 \mathrm{~Hz}, 1.66 \mathrm{H}, \mathrm{H}^{\mathrm{G}}\right), 4.19-4.09\left(\mathrm{~m}, 11.09 \mathrm{H}, \mathrm{H}^{\mathrm{A}}, \mathrm{H} 5^{\mathrm{B}}, \mathrm{H} 5^{\mathrm{C}}, \mathrm{H} 2^{\mathrm{E}}, \mathrm{H} 4^{\mathrm{E}}, \mathrm{H} 2^{\mathrm{F}}, \mathrm{H} 2^{\mathrm{G}}\right), 4.01-4.00$ $\left(\mathrm{m}, 0.61 \mathrm{H}, \mathrm{H}^{\mathrm{A}}{ }_{\alpha}\right), 3.98\left(\mathrm{dd}, J=5.8,3.2 \mathrm{~Hz}, 1.14 \mathrm{H}, \mathrm{H}^{\mathrm{E}}\right), 3.96\left(\mathrm{dd}, J=5.5,2.4 \mathrm{~Hz}, 1.62 \mathrm{H}, \mathrm{H}^{\mathrm{F}}\right)$, 3.94-3.92 (m, 3.49H, H5 $\left.{ }^{\mathrm{D}}, \mathrm{H}^{\mathrm{G}}\right), 3.89$ (dd, $\left.J=9.1,4.9 \mathrm{~Hz}, 1.58 \mathrm{H}, \mathrm{H}^{\mathrm{B}}\right), 3.86-3.73(\mathrm{~m}, 19.09 \mathrm{H}$, $\left.\mathrm{H}^{\mathrm{A}}{ }_{\alpha}, \mathrm{H} 4{ }^{\mathrm{A}}{ }_{\beta}, \mathrm{H} 5^{\mathrm{A}}{ }_{\alpha}, \mathrm{H} 5^{\prime}{ }_{\alpha}, \mathrm{H}^{\mathrm{B}}, \mathrm{H}^{\mathrm{C}}, \mathrm{H} 4^{\mathrm{C}}, \mathrm{H} 5^{\mathrm{E}}, \mathrm{H} 5^{\mathrm{E}}, \mathrm{H} 5^{\mathrm{F}}, \mathrm{H} 5^{\prime} \mathrm{F}, \mathrm{H} 5^{\mathrm{G}}, \mathrm{H} 5^{\circ} \mathrm{G}\right), 3.63-3.57$ (m, 4.95H, H2 $\left.{ }_{\alpha}^{\mathrm{A}}, \mathrm{H}^{\mathrm{A}}{ }_{\beta}, \mathrm{H} 2^{\mathrm{B}}, \mathrm{H} 4^{\mathrm{D}}\right), 3.47-3.42\left(\mathrm{~m}, 6.09 \mathrm{H}, \mathrm{H} 5^{{ }^{\mathrm{A}}}{ }_{\beta}, \mathrm{H}^{\mathrm{B}}, \mathrm{H} 2^{\mathrm{C}}, \mathrm{H} 3^{\mathrm{D}}\right), 3.40-3.37$ (m, $\left.1.75 \mathrm{H}, \mathrm{H} 5^{\circ} \mathrm{C}\right), 3.31-3.25\left(\mathrm{~m}, 4.64 \mathrm{H}, \mathrm{H} 2^{\mathrm{A}}{ }_{\beta}, \mathrm{H} 2^{\mathrm{D}}, \mathrm{H} 5^{\circ} \mathrm{D}\right) .{ }^{13} \mathrm{C}\left\{{ }^{1} \mathrm{H}\right\} \mathrm{NMR}\left(201 \mathrm{MHz}, \mathrm{D}_{2} \mathrm{O}\right) \delta 108.7$, $108.7\left(\mathrm{C1}^{\mathrm{E}}\right), 108.1\left(\mathrm{C}^{\mathrm{F}}\right), 107.6\left(\mathrm{C}^{\mathrm{G}}\right), 101.4\left(\mathrm{C}^{\mathrm{D}}\right), 101.2\left(\mathrm{C}^{\mathrm{C}}\right), 99.9,99.8\left(\mathrm{C1}^{\mathrm{B}}\right), 96.5\left(\mathrm{C} 1^{\mathrm{A}}\right)$, $92.0\left(\mathrm{C1}_{\alpha}^{\mathrm{A}}\right), 84.8\left(\mathrm{C}^{\mathrm{G}}\right), 84.4,84.3\left(\mathrm{C}^{\mathrm{E}}\right), 84.3\left(\mathrm{C} 4^{\mathrm{F}}\right), 81.2,81.2\left(\mathrm{C} 2^{\mathrm{E}}\right), 81.0\left(\mathrm{C} 2^{\mathrm{F}}\right), 80.7\left(\mathrm{C} 2^{\mathrm{G}}\right)$, 78.6, 78.6 $\left(\mathrm{C}^{\mathrm{B}}\right)$, 77.6, 77.5 $\left(\mathrm{C} 3^{\mathrm{B}}\right), 77.2,77.2,77.2\left(\mathrm{C} 3^{\mathrm{C}}, \mathrm{C} 3^{\mathrm{F}}, \mathrm{C}^{\mathrm{G}}\right), 76.6\left(\mathrm{C} 3^{\mathrm{E}}\right), 76.5\left(\mathrm{C} 4^{\mathrm{A}}{ }_{\alpha}\right)$, $75.9\left(\mathrm{C}^{\mathrm{A}}{ }_{\beta}\right), 75.8,75.6\left(\mathrm{C} 3^{\mathrm{D}}\right), 74.0,73.9,73.8\left(\mathrm{C}^{\mathrm{A}}{ }_{\beta}, \mathrm{C}^{\mathrm{A}}{ }_{\beta}, \mathrm{C} 4^{\mathrm{B}}\right), 73.7,73.6\left(\mathrm{C} 2^{\mathrm{C}}, \mathrm{C}^{\mathrm{C}}\right), 72.9$ $\left(\mathrm{C} 2^{\mathrm{D}}\right), 71.4\left(\mathrm{C} 2^{\mathrm{A}}{ }_{\alpha}\right), 71.0\left(\mathrm{C} 3^{\mathrm{A}}{ }_{\alpha}\right), 69.2\left(\mathrm{C} 4^{\mathrm{D}}\right), 65.1\left(\mathrm{C}^{\mathrm{D}}\right), 62.9,62.7\left(\mathrm{C}^{\mathrm{A}}{ }_{\beta}, \mathrm{C} 5^{\mathrm{C}}\right), 62.5\left(\mathrm{C} 5^{\mathrm{B}}\right)$, 62.5, 61.3, 61.2, 61.1, $61.0\left(\mathrm{C}^{\mathrm{E}}, \mathrm{C} 5^{\mathrm{F}}, \mathrm{C} 5^{\mathrm{G}}\right), 58.7\left(\mathrm{C}^{\mathrm{A}}{ }_{\alpha}\right)$. HRMS: $m / z$ calcd for $\mathrm{C}_{35} \mathrm{H}_{58} \mathrm{O}_{29} \mathrm{Na}$ [M $+\mathrm{Na}]^{+}$965.2956, found 965.2965.

$\beta$-D-Xylopyranosyl-( $1 \rightarrow 4)$-[ $\alpha$-L-arabinofuranosyl- $(1 \rightarrow 2)]$-[ $\alpha$-L-arabinofuranosyl- $(1 \rightarrow 3)]-\beta$ - $D$ -

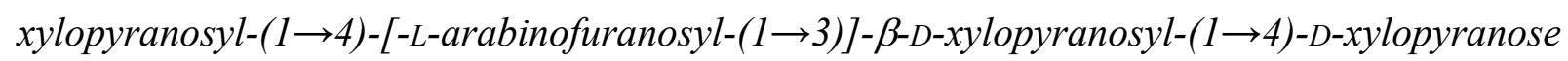
(3). General procedure $\mathrm{G}$ with partially deprotected heptasaccharide 34 (10.6 mg, $0.010 \mathrm{mmol}$ ), 2,6-lutidine ( $3 \mu \mathrm{L}, 0.015 \mathrm{mmol})$ and NBS (5.5 mg, $0.031 \mathrm{mmol})$. Reaction time $1.5 \mathrm{~h}$. The 
product was obtained as a white powder $(8.5 \mathrm{mg}, 88 \%) . \alpha / \beta$ ratio $0.6: 1 . R_{\mathrm{f}} 0.33$

(EtOAc/MeOH/ $\mathrm{H}_{2} \mathrm{O} / \mathrm{AcOH}$ 6:3:0.8:0.2). ${ }^{1} \mathrm{H}$ NMR $\left(800 \mathrm{MHz}, \mathrm{D}_{2} \mathrm{O}\right) \delta 5.44-5.44(\mathrm{~m}, 1.57 \mathrm{H}$, $\left.\mathrm{H} 1^{\mathrm{E}}\right), 5.30\left(\mathrm{~s}, 1.56 \mathrm{H}, \mathrm{H} 1^{\mathrm{G}}\right), 5.25\left(\mathrm{~s}, 1.55 \mathrm{H}, \mathrm{H} 1^{\mathrm{F}}\right), 5.21\left(\mathrm{~d}, J=3.6 \mathrm{~Hz}, 1 \mathrm{H}, \mathrm{H} 1_{\alpha}^{\mathrm{A}}\right), 4.62-4.60(\mathrm{~m}$, $\left.2.70 \mathrm{H}, \mathrm{H} 1^{\mathrm{A}}{ }_{\beta}, \mathrm{H} 1^{\mathrm{C}}\right), 4.52-4.51\left(\mathrm{~m}, 1.58 \mathrm{H}, \mathrm{H} 1^{\mathrm{B}}\right), 4.45\left(\mathrm{~d}, J=7.8 \mathrm{~Hz}, 1.58 \mathrm{H}, \mathrm{H} 1^{\mathrm{D}}\right), 4.34-4.31(\mathrm{~m}$, 3.29H, H4 $\left.4^{\mathrm{E}}, \mathrm{H} 4^{\mathrm{G}}\right), 4.19-4.18\left(\mathrm{~m}, 3.20 \mathrm{H}, \mathrm{H} 2^{\mathrm{E}}, \mathrm{H} 2^{\mathrm{F}}\right), 4.17-4.15\left(\mathrm{~m}, 5.36 \mathrm{H}, \mathrm{H} 5^{\mathrm{B}}, \mathrm{H} 2^{\mathrm{F}}, \mathrm{H} 4^{\mathrm{F}}\right), 4.10$ $\left(\mathrm{dd}, J=11.9,4.2 \mathrm{~Hz}, 1.60 \mathrm{H}, \mathrm{H}^{\mathrm{C}}\right), 4.07\left(\mathrm{dd}, J=11.8,5.4 \mathrm{~Hz}, 1.09 \mathrm{H}, \mathrm{H} 5^{\mathrm{A}}{ }_{\beta}\right), 4.00(\mathrm{dd}, J=5.9$, 3.1 Hz, 1.57H, H3 $\left.3^{\mathrm{F}}\right), 3.96-3.93\left(\mathrm{~m}, 5.17 \mathrm{H}, \mathrm{H}^{\mathrm{D}}, \mathrm{H}^{\mathrm{E}}, \mathrm{H}^{\mathrm{G}}\right), 3.87-3.73\left(\mathrm{~m}, 22.09 \mathrm{H}, \mathrm{H}^{\mathrm{A}}{ }_{\alpha}, \mathrm{H}^{\mathrm{A}}{ }_{\beta}\right.$, $\left.\mathrm{H}_{4}^{\mathrm{A}}{ }_{\alpha}, \mathrm{H} 5^{\mathrm{A}}{ }_{\alpha}, \mathrm{H} 5^{{ }^{\mathrm{A}}}{ }_{\alpha}, \mathrm{H}^{\mathrm{B}}, \mathrm{H} 4^{\mathrm{B}}, \mathrm{H} 3^{\mathrm{C}}, \mathrm{H}^{\mathrm{C}}\right), 3.63-3.55\left(\mathrm{~m}, 5.31 \mathrm{H}, \mathrm{H}^{2}{ }_{\alpha}^{\mathrm{A}}, \mathrm{H}^{\mathrm{A}}{ }_{\beta}, \mathrm{H}^{\mathrm{C}}, \mathrm{H} 4^{\mathrm{D}}\right)$, 3.50-3.45 (m, 3.52H, H2 $\left.{ }^{\mathrm{B}}, \mathrm{H} 5^{\mathrm{B}}{ }^{\mathrm{B}}\right), 3.43\left(\mathrm{t}, J=9.3 \mathrm{~Hz}, 1.94 \mathrm{H}, \mathrm{H}^{\mathrm{D}}\right), 3.41-3.38\left(\mathrm{~m}, 2.99 \mathrm{H}, \mathrm{H}^{\mathrm{C}}\right.$, $\mathrm{H} 5^{\circ}$ ), $3.30-3.25\left(\mathrm{~m}, 4.54 \mathrm{H}, \mathrm{H} 2^{\mathrm{A}}{ }_{\beta}, \mathrm{H} 2^{\mathrm{D}}, \mathrm{H} 5^{\circ}\right) .{ }^{13} \mathrm{C}\left\{{ }^{1} \mathrm{H}\right\} \mathrm{NMR}\left(201 \mathrm{MHz}, \mathrm{D}_{2} \mathrm{O}\right) \delta 108.7\left(\mathrm{Cl}^{\mathrm{F}}\right)$, 108.0 $\left(\mathrm{Cl}^{\mathrm{G}}\right), 107.5\left(\mathrm{Cl}^{\mathrm{E}}\right), 101.6\left(\mathrm{Cl}^{\mathrm{B}}\right), 101.3\left(\mathrm{Cl}^{\mathrm{D}}\right), 99.8\left(\mathrm{C1}^{\mathrm{C}}\right), 96.5\left(\mathrm{Cl}^{\mathrm{A}}{ }_{\beta}\right), 92.0\left(\mathrm{Cl}^{\mathrm{A}}{ }_{\alpha}\right), 85.0$ $\left(\mathrm{C} 4^{\mathrm{E}}\right), 84.3,84.2\left(\mathrm{C} 4^{\mathrm{F}}, \mathrm{C} 4^{\mathrm{G}}\right), 81.3\left(\mathrm{C} 2^{\mathrm{F}}\right), 80.9\left(\mathrm{C}^{\mathrm{G}}\right), 80.5\left(\mathrm{C} 2^{\mathrm{E}}\right), 78.7\left(\mathrm{C} 2^{\mathrm{C}}\right), 77.7\left(\mathrm{C} 3^{\mathrm{C}}\right), 77.3$, $77.2\left(\mathrm{C3}^{\mathrm{E}}, \mathrm{C}^{\mathrm{G}}\right), 77.0,77.0\left(\mathrm{C}^{\mathrm{B}}\right), 76.7\left(\mathrm{C}^{\mathrm{F}}\right), 76.6\left(\mathrm{C}^{\mathrm{A}}{ }_{\alpha}\right), 76.4\left(\mathrm{C}^{\mathrm{A}}{ }_{\beta}\right), 75.6\left(\mathrm{C}^{\mathrm{D}}\right), 74.0,73.9$ $\left(\mathrm{C} 2^{\mathrm{A}}{ }_{\beta}, \mathrm{C} 3^{\mathrm{A}}{ }_{\beta}\right), 73.6\left(\mathrm{C} 4^{\mathrm{C}}\right), 73.3\left(\mathrm{C} 2^{\mathrm{B}}, \mathrm{C} 4^{\mathrm{B}}\right), 73.0\left(\mathrm{C} 2^{\mathrm{D}}\right), 71.3\left(\mathrm{C}^{\mathrm{A}}{ }_{\alpha}\right), 70.9\left(\mathrm{C}^{\mathrm{A}}{ }_{\alpha}\right), 69.2\left(\mathrm{C} 4^{\mathrm{D}}\right)$, $65.1\left(\mathrm{C}^{\mathrm{D}}\right), 62.9,62.8,62.8,62.6\left(\mathrm{C}^{\mathrm{C}}\right)\left(\mathrm{C}^{\mathrm{A}}{ }_{\beta}, \mathrm{C}^{\mathrm{B}}, \mathrm{C}^{\mathrm{C}}\right), 61.4,61.1,61.1\left(\mathrm{C}^{\mathrm{E}}, \mathrm{C}^{\mathrm{F}}, \mathrm{C} 5^{\mathrm{G}}\right), 58.8$ $\left(\mathrm{C} 5^{\mathrm{A}}{ }_{\alpha}\right)$. HRMS: $m / z$ calcd for $\mathrm{C}_{35} \mathrm{H}_{58} \mathrm{O}_{29} \mathrm{Na}[\mathrm{M}+\mathrm{Na}]^{+}$965.2956, found 965.2966.

Supporting Information. Copies of ${ }^{1} \mathrm{H}$ and ${ }^{13} \mathrm{C}\left\{{ }^{1} \mathrm{H}\right\}$ NMR spectra for the prepared compounds as well as 2D NMR spectra for compounds $\mathbf{1}-\mathbf{3}$. This material is available free of charge via the Internet at http://pubs.acs.org.

\section{ACKNOWLEDGMENT}


We thank the Danish Council for Strategic Research for financial support (SET4Future project, grant 0603-00463B). In addition, The NMR Center • DTU and the Villum Foundation are acknowledged for access to the $800 \mathrm{MHz}$ spectrometer.

\section{References}

1) Naidu, D. S.; Hlangothi, S. P.; John, M. J. Bio-based products from xylan: A review. Carbohydr. Polym. 2018, 179, 28-41.

2) Zhang, Z.; Smith, C.; Li, W. Extraction and modification technology of arabinoxylans from cereal by-products: A critical review. Food Res. Int. 2014, 65, 423-436.

3) (a) Chen, Z.; Li, S.; Fu, Y.; Li, C.; Chen, D.; Chen, H. Arabinoxylan structural characteristics, interaction with gut microbiota and potential health functions. J. Funct. Foods 2019, 54, 536-551. (b) Mendis, M.; Leclerc, E.; Simsek, S. Arabinoxylans, gut microbiota and immunity. Carbohydr. Polym. 2016, 139, 159-166.

4) (a) Stoklosa, R. J.; Latona, R. J.; Bonnaillie, L. M.; Yadav, M. P. Evaluation of arabinoxylan isolated from sorghum bran, biomass and bagasse for film formation. Carbohydr. Polym. 2019, 213, 382-392. (b) Yu, L.; Yakubov, G. E.; Gilbert, E. P.; Sewell, K.; van de Meene, A. M. L.; Stokes, J. R. Multi-scale assembly of hydrogels formed by highly branched arabinoxylans from Plantago ovata seed mucilage studied by USANS/SANS and rheology. Carbohydr. Polym. 2019, 207, 333-342.

5) (a) Biely, P.; Singh, S.; Puchart, V. Towards enzymatic breakdown of complex plant xylan structures: State of the art. Biotechnol. Adv. 2016, 34, 1260-1274. (b) Lagaert, S.; Pollet, A.; Courtin, C. M.; Volckaert, G. $\beta$-Xylosidases and $\alpha$-L-arabinofuranosidases: Accessory enzymes for arabinoxylan degradation. Biotechnol. Adv. 2014, 32, 316-332. 
6) Wilkens, C.; Andersen, S.; Dumon, C.; Berrin, J.-G.; Svensson, B. GH62

arabinofuranosidases: Structure, function and applications. Biotechnol. Adv. 2017, 35, 792-804.

7) (a) Mathew, S.; Karlsson, E. N.; Adlercreutz, P. Extraction of soluble arabinoxylan from enzymatically pretreated wheat bran and production of short xylo-oligosaccharides and arabinoxylooligosaccharides from arabinoxylan by glycoside hydrolase family 10 and 11 endoxylanases. J. Biotechnol. 2017, 260, 53-61. (b) Mechelke, M.; Koeck, D. E.; Broeker, J.; Roessler, B.; Krabichler, F.; Schwartz, W. H.; Zverlov, V. V.; Liebl, W. Characterization of the arabinoxylan-degrading machinery of the thermophilic bacterium Herbinix hemicellulosilytica - Six new xylanases, three arabinofuranosidases and one xylosidase. $J$. Biotechnol. 2017, 257, 122-130. (c) McCleary, B. V.; McKie, V. A.; Draga, A.; Rooney, E.; Mangan, D.; Larkin, J. Hydrolysis of wheat flour arabinoxylan, acid-debranched wheat flour arabinoxylan and arabino-xylo-oligosaccharides by $\beta$-xylanase, $\alpha$-Larabinofuranosidase and $\beta$-xylosidase. Carbohydr. Res. 2015, 407, 79-96.

8) (a) Utille, J.-P.; Jeacomine, I. Synthesis of a library of allyl $\alpha$-L-arabinofuranosyl- $\alpha$ - or $\beta$ D-xylopyranosides; route to higher oligomers. Carbohydr. Res. 2007, 342, 2649-2656. (b) Hirsch, J.; Petráková, E.; Schraml, J. Stereoselective synthesis and ${ }^{13}$ C-N.M.R. spectra of two isomeric methyl $\beta$-glycosides of trisaccharides related to arabinoxylan. Carbohydr. Res. 1984, 131, 219-226.

9) (a) Senf, D.; Ruprecht, C.; de Kruijff, G. H. M.; Simonetti, S. O.; Schuhmacher, F.; Seeberger, P. H.; Pfrengle, F. Active Site Mapping of Xylan-Deconstructing Enzymes with Arabinoxylan Oligosaccharides Produced by Automated Glycan Assembly. Chem. Eur. J. 2017, 23, 3197-3205. (b) Schmidt, D.; Schuhmacher, F.; Geissner, A.; Seeberger, P. H.; 
Pfrengle, F. Automated Synthesis of Arabinoxylan-Oligosaccharides Enables Characterization of Antibodies that Recognize Plant Cell Wall Glycans. Chem. Eur. J. 2015, 21, 5709-5713.

10) Kinnaert, C.; Daugaard, M.; Nami, F.; Clausen, M. H. Chemical Synthesis of Oligosaccharides Related to the Cell Walls of Plants and Algae. Chem. Rev. 2017, 117, $11337-11405$.

11) Takeo, K.; Ohguchi, Y.; Hasegawa, R.; Kitamura, S. Synthesis of ( $1 \rightarrow 4)-\beta$-D-xylooligosaccharides of dp 4-10 by a blockwise approach. Carbohydr. Res. 1995, 278, 301-313.

12) Pedersen, M. J.; Madsen, R.; Clausen, M. H. Iridium catalysis: reductive conversion of glucan to xylan. Chem. Commun. 2018, 54, 952-955.

13) Bonora, B.; Boos, I.; Clausen, M. H. Convergent strategy for the synthesis of $S$-linked oligoxylans. Carbohydr. Res. 2017, 443-444, 53-57.

14) (a) Zakharova, A. N.; Madsen, R.; Clausen, M. H. Synthesis of a Backbone Hexasaccharide Fragment of the Pectic Polysaccharide Rhamnogalacturonan I. Org. Lett. 2013, 15, 1826-1829. (b) Clausen, M. H.; Madsen, R. Synthesis of Hexasaccharide Fragments of Pectin. Chem. Eur. J. 2003, 9, 3821-3832. (c) Clausen, M. H.; Jørgensen, M. R.; Thorsen, J.; Madsen, R. A strategy for chemical synthesis of selectively methylesterified oligomers of galacturonic acid. J. Chem. Soc., Perkin Trans. 1 2001, 543-551.

15) (a) Yang, B.; Yang, W.; Ramadan, S.; Huang, X. Pre-Activation-Based Stereoselective Glycosylations. Eur. J. Org. Chem. 2018, 1075-1096. (b) Yang, W.; Yang, B.; Ramadan, S.; Huang, X. Preactivation-based chemoselective glycosylations: A powerful strategy for oligosaccharide assembly. Beilstein J. Org. Chem. 2017, 13, 2094-2114. 
16) Huang, X.; Huang, L.; Wang, H.; Ye, X.-S. Iterative One-Pot Synthesis of Oligosaccharides. Angew. Chem. Int. Ed. 2004, 43, 5221-5224.

17) For recent examples, see: (a) Zhang, H.; Shao, L.; Wang, X.; Zhang, Y.; Guo, Z.; Gao, J. One-Pot Synthesis of the Repeating Unit of Type VII Group B Streptococcus Polysaccharide and the Dimer. Org. Lett. 2019, 21, 2374-2377. (b) Zhang, Y.; Zhou, S.; Wang, X.; Zhang, H.; Guo, Z.; Gao, J. A new method for $\alpha$-specific glucosylation and its application to the one-pot synthesis of a branched $\alpha$-glucan. Org. Chem. Front. 2019, 6, 762-772. (c) Wang, P.; Lo Cascio F.; Gao, J.; Kayed, R.; Huang, X. Binding and neurotoxicity mitigation of toxic tau oligomers by synthetic heparin like oligosaccharides. Chem. Commun. 2018, 54, 10120-10123. (d) Xiong, C.; Feng, S.; Qiao, Y.; Guo, Z.; Gu, G. Synthesis and Immunological Studies of Oligosaccharides that Consist of the Repeating Unit of Streptococcus pneumoniae Serotype 3 Capsular Polysaccharide. Chem. Eur. J. 2018, 24, 8205-8216.

18) Wu, Y.; Xiong, D.-C.; Chen, S.-C.; Wang, Y.-S.; Ye, X.-S. Total synthesis of mycobacterial arabinogalactan containing 92 monosaccharide units. Nat. Commun. 2017, 8, 14851.

19) (a) Andersen, M. C. F.; Kračun, S. K.; Rydahl, M. G.; Willats, W. G. T.; Clausen, M. H. Synthesis of $\beta$-1,4-Linked Galactan Side-Chains of Rhamnogalacturonan I. Chem. Eur. J. 2016, 22, 11543-11548. (b) Dai, Y.; Yu, B. Total synthesis of astrosterioside A, an antiinflammatory asterosaponin. Chem. Commun. 2015, 51, 13826-13829.

20) López, R.; Fernández-Mayorales, A. Enzymatic $\beta$-Galactosidation of Modified Monosaccharides: Study of the Enzyme Selectivty for the Acceptor and Its Application to the Synthesis of Disaccharides. J. Org. Chem. 1994, 59, 737-745. 
21) Thorsheim, K.; Siegbahn, A.; Johnsson, R. E.; Stålbrand, H.; Manner, S.; Widmalm, G.; Ellervik, U. Chemistry of xylopyranosides. Carbohydr. Res. 2015, 418, 65-88.

22) Rio, S.; Beau, J.-M.; Jacquinet, J.-C. Synthesis of glycopeptides from the carbohydrateprotein linkage region of proteoglycans. Carbohydr. Res. 1991, 219, 71-90.

23) Yang, B.; Yoshida, K.; Yin, Z.; Dai, H.; Kavunja, H.; El-Dakdouki, M. H.; Sungsuwan, S.; Dulaney, S. B.; Huang, X. Chemical Synthesis of a Heparan Sulfate Glycopeptide: Syndecan-1. Angew. Chem. Int. Ed. 2012, 51, 10185-10189.

24) Andersen, S. M.; Heuckendorff, M.; Jensen, H. J. 3-(Dimethylamino)-1-propylamine: A Cheap and Versatile Reagent for Removal of Byproducts in Carbohydrate Chemistry. Org. Lett. 2015, 17, 944-947.

25) This procedure has previously been used for regioselective benzoylation of ethyl 1thiohexopyranosides, see: Garegg, P. J.; Kvarström, I.; Niklasson, A.; Niklasson, G.; Svensson, S. C. T. Partial Substitution of Thioglycosides by Phase Transfer Catalyzed Benzoylation and Benzylation. J. Carhohydr. Chem. 1993, 12, 933-953.

26) Martichonok, V.; Whitesides, G. M. Stereoselective $\alpha$-Sialylation with Sialyl Xanthate and Phenylsulfenyl Triflate as a Promoter. J. Org. Chem. 1996, 61, 1702-1706.

27) Tatai, J.; Fügedi, P. A New, Powerful Glycosylation Method: Activation of Thioglycosides with Dimethyl Disulfide-Triflic Anhydride. Org. Lett. 2007, 9, 4647-4650.

28) Crich, D.; Smith, M. 1-Benzenesulfinyl Piperidine/Trifluoromethanesulfonic Anhydride: A Potent Combination of Shelf-Stable Reagents for the Low-Temperature Conversion of Thioglycosides to Glycosyl Triflates and for the Formation of Diverse Glycosidic Linkages. J. Am. Chem. Soc. 2001, 123, 9015-9020. 
29) Crich, D.; Cai, F.; Yang, F. A stable, commercially available sulfenyl chloride for the activation of thioglycosides in conjunction with silver trifluoromethanesulfonate. Carbohydr. Res. 2008, 343, 1858-1862.

30) Zeng, Y.; Wang, Z.; Whitfield, D.; Huang, X. Installation of Electron-Donating Protective Groups, a Strategy for Glycosylating Unreactive Thioglycosyl Acceptors using the Preactivation-Based Glycosylation Method. J. Org. Chem. 2008, 73, 7952-7962.

31) Christensen, H. M.; Oscarson, S.; Jensen, H. H. Common side reactions of the glycosyl donor in chemical glycosylation. Carbohydr. Res. 2015, 408, 51-95.

32) For a previous example of aglycon transfer during the pre-activation protocol, see: Huang, L.; Wang, Z.; Li, X.; Ye, X.-s.; Huang, X. Iterative one-pot syntheses of chitotetroses. Carbohydr. Res. 2006, 341, 1669-1679.

33) Crich, D.; Dai, Z.; Gastaldi, S. On the Role Neighboring Group Participation and Ortho Esters in $\beta$-Xylosylation: ${ }^{13} \mathrm{C}$ NMR Observation of a Bridging 2-Phenyl-1,3-dioxalenium Ion. J. Org. Chem. 1999, 64, 5224-5229.

34) Crich, D.; Smith, M.; Yao, Q.; Picione, J. 2,4,6-Tri-tert-butylpyrimidine (TTBP): A Cost Effective, Readily Available Alternative to the Hindered Base 2,6-Di-tert-butylpyridine and its 4-Substituted Derivatives in Glycosylation and Other Reactions. Synthesis 2001, $323-326$.

35) van Rijssel, E. R.; Goumans, T. P. M.; Lodder, G.; Overkleeft, H. S.; van der Marel, G. A.; Codée, J. D. C. Chiral Pyrroline-Based Ugi-Three-Component Reactions Are under Kinetic Control. Org. Lett. 2013, 15, 3026-3029.

36) Bock, K.; Pedersen, C. A Study of ${ }^{13} \mathrm{CH}$ Coupling Constants in Pentopyranoses and Some of their Derivatives. Acta Chem. Scand. 1975, B29, 258-264. 
37) Mizutani, K.; Kasai, R.; Nakamura, M.; Tanaka, O. N.M.R. Spectral Study of $\alpha$ - and $\beta$-LArabinofuranosides. Carbohydr. Res. 1989, 185, 27-38.

38) Motawia, M. S.; Marcussen, J.; Møller, B. L. A General Method Based on the Use of $N$ Bromosuccinimide for Removal of the Thiophenyl Group at the Anomeric Position to Generate A Reducing Sugar with the Original Protecting Groups Still Present. $J$. Carbohydr. Chem. 1995, 14, 1279-1294.

39) For Zemplén deprotection of perbenzoylated gluco-, manno- and galactopyranose in quantitative yields, see: Ren, B.; Wang, M.; Liu, J.; Ge, J.; Zhang, X.; Dong, H. Zemplén transesterification: a name reaction that has misled us for 90 years. Green Chem. 2015, 17, 1390-1394.

40) The term peeling originates from the observed degradation of polysaccharides during the alkaline pulping and bleaching of wood, see: (a) Berglund, J.; Azhar, S.; Lawoko, M.; Lindström, M.; Vilaplana, F.; Wohlert, J.; Henriksson, G. The structure of galactoglucomannan impacts the degradation under alkaline conditions. Cellulose 2019, 26, 2155-2175. (b) Wang, Y.; Azhar, S.; Lindström, M. E.; Henriksson, G. Stabilization of Polysaccharides During Alkaline Pre-Treatment of Wood Combined with EnzymeSupported Extractions in a Biorefinery. J. Wood Chem. Technol. 2015, 35, 91-101.

41) Andersen, M. C. F.; Boos, I.; Ruprecht, C.; Willats, W. G. T.; Pfrengle, F.; Clausen, M. H. Synthesis and Application of Branched Type II Arabinogalactans. J. Org. Chem. 2017, 82, 12066-12084.

42) Pedersen, D. S.; Rosenbohm, C. Dry Column Vacuum Chromatography. Synthesis 2001, 2431-2434. 
43) Kongkathip, B.; Kongkathip, N.; Rujirawanich, J. New Strategy for Synthesis of the Disaccharide Moiety of the Highly Potent Anticancer Natural Product OSW-1. Synth. Commun. 2014, 44, 2248-2255.

44) Crich, D.; Dai, Z. Direct Synthesis of $\beta$-Mannosides. Synthesis of $\beta$-D-Xyl- $(1 \rightarrow 2)-\beta-D-$ Man-(1 $\rightarrow 4)-\alpha-D-G l c-O M e:$ a Trisaccharide Component of the Hyriopsis schlegelii Glycosphingolipid. Formation of an Orthoester from a Xylopyranosyl Sulfoxide. Tetrahedron 1999, 55, 1569-1580.

45) Phanumartwiwath, A.; Hornsby, T. W.; Jamalis, J.; Bailey, C. D.; Willis, C. L. Silyl Migrations in D-Xylose Derivatives: Total Synthesis of a Marine Quinoline Alkaloid. Org. Lett. 2013, 15, 5734-5737. 